MUON DIFFUSION IN THE METAL IIYDRIDES LaN ${ }_{5}-6$ AND $\beta-P$ dH $x$

F.N. Gygax, A. Hintermann, W. Ruegg, A. Schenck, W. Studer and A.J. van der Wal

Institute for Medium Energy Fhysics, ETH-Zurich, c/o SIN, CII-5234 Villigen, Switzerland

end

F. Stucki and I. Schlapbach

Laboratory for Solid State Physics, ETH-Zurich, CH-8093 Zurich, Switzerland

Contribution to the International Meeting on Hydrogen in Metals, Wroclaw, Poland, September 1983 
$n$ bstract

zero and transverse field $\mu^{+} S R$ measurements have been

performed in $\mathrm{LaN}_{5} \mathrm{H}_{6}$ and in $\mathrm{PdH}_{x}$ ( $x$ from 0.59 to 0.86 )

between $16 \mathrm{~K}$ and roon temperature. The $\mu^{+}$

depolarization is due to the spread in nuclear dipole

fields originating predominantly from the protons.

Motional averaging is caused by the combined motion of protons and the $\mu^{+}$. The results show a surprising low correlation rate $1 / \tau_{c}$, which points to a highly correlated $\mu^{+}-$proton diffusion or to $\mu^{+}$trapping within regions of largely immobile hydrogen

configurations. In $\mathrm{LaNi}_{5} \mathrm{H}_{6}$ muon trapping or a localized $\mu^{+}$jump mode could be considered. In that sample a linear decrease of the second moment with temperature between $20 \mathrm{~K}$ and $120 \mathrm{~K}$ is measured, in contrast to the unchanged relaxation observed by proton NMI.

\section{DISCLAIMER}

This report was prepared as an account of work sponsored by an agency of the United States Government. Neither the United States Government nor any agency thereof, nor any of their employees, makes any warranty, express or implied, or assumes any legal liability or responsibility for the accuracy, completeness, or usefulness of any information, apparatus, product, of process disclosed, or represents that its use would not infringe privately owned rights. Reference herein to any specific commercial producl, process, or service by trade name, trademark, manufacturer, or otherwise does not necessarily constitute or imply its endorsement, recommendation, or favoring by the United States Government or eny agency thereof. Tho views and opinions of authors expressed herein do not necessarily state or reflect those of the United States Government or any agency thereor. 


\section{Introduction}

Upin reluxation experiments with positive muons in metal hydrides offer the possibility to compare directly the $\mu^{+}$diffusion with the proton diffusion. The proton diffusion is usually well known from other experiments.

In systems like $\mathrm{IaNi}_{5} \mathrm{H}_{6}$ or $\beta-\mathrm{PdH}_{x}$ the $\mu^{+}$ depolarization is predominantly caused by the spread in nuclear dipole fields originating from the protons. The $\mu^{+}$relaxation function is as usual characterized by two parameters: (i) $\mathrm{M}_{2}$, the second moment of the dipolar field spread, mainly determined by the structural arrangement of protons around the $\mu^{+}$, (ii) $\tau_{c}$, the correlation time originating from the fluctuations in the dipolar field components due to the motion of both $\mu^{+}$and proton.

Assuming an uncorrelated motion of muon and protons, the correlation rate $1 / \tau_{c}$ can be written as

$$
\frac{1}{\tau_{c}}=\frac{1}{\tau_{\mu}}+\frac{1}{\tau_{p}},
$$

where $1 / \tau_{\mu}$ is the $\mu^{+}$jump rate and $1 / \tau_{p}$ the proton jump rate. Irrespective of the motional behaviour of the muon, one would then expect to find

$$
\frac{1}{\tau_{c}} \geqslant \frac{1}{\tau_{p}} \text {, }
$$


i.e. that the rate $1 / \tau_{c}$ would never be slower than the proton jump rate. For $\operatorname{LaNi}_{5} \mathrm{H}_{6}$ and $\beta-\mathrm{P}_{\mathrm{d}} \mathrm{H}_{x} 1 / \tau_{p}$ is known from NMR and neutron diffraction measurements $[1-9]$.

2. Laldi ${ }_{5}=6$

The sample used for the $\mu$ SR experiments was only hydrogenated two times in order to avoid $\mathrm{Ni}$ precipitation on the surface which could lead to an artificial increase of the $\mu^{+}$relaxation rate [10]. The resulting powdery material was sealed in a sphericai quartz ampoule.

Fig. 1 shows the measured temperature dependence of the muon depolarization rate $\Lambda(1 / \Lambda$ is the time for which the polarization decreases to $1 / \mathrm{e})$. Measurements were performed in transverse field (200 G) as well as in zero field, both techniques yielding identical results. (For the zero field case, Kubo-Toyabe functions are taken to fit the data. The dipolar field spreads $\sigma_{z F}$ have to be scaled down by a factor $2 / 5$ for direct comparison with the transverse field damping rates, according to theory.) The scaled line width of a proton NMR experiment by llalstead [1] is displayed for comparison in fig. 1. (The scale of the NMR line width has been determined from the square root of the reported second moment times $2 / 3$ to account for the difference between the like spin 
case $[p-p]$ and the unlike spin case $[\mu-p]$.

For $T<120 \mathrm{~K}$ one sees that the NMR damping is constant (which ia confirmed by Barnes et al, [2]), whereas the muon depolarization rate decreases gently to a" plateau value of $0.13 \mathrm{ks}^{-1}$ around $150 \mathrm{~K}$ (dotted level in fig. 1). No diffusion occurs below $150 \mathrm{~K}$, all zero field measurements are clearly best fitted by a static Kubo-Toyabe function.

Above $160 \mathrm{~K}$ motional averaging sets in and the data are analysed either with the usual transverse field motional narrowing function or with the dynamic Kubo-Toyabe function, using $\omega^{\circ}=0.13 \mu^{-1}$ (or $\left.\sigma_{z F}=5 / 2 \times 0.13 \mu \mathrm{s}^{-1}\right)$. Fig. 2 shows the results for $1 / \tau_{c}$ plotted in logarithmic scale vs. $1 / T$. The straight solid line in fig. 2 represents corresponding data from a proton NMR study by Halstead et al. [3] . These data are in good agreement with other NMR and neutron diffraction experiments $[1,4,5]$.

In the temperature region of diffusive motion, three striking differences between $\mu^{+}$and proton behaviour are apparent: .

(i) The muon datia differ from an Arrhenius like behaviour for $T>250 \mathrm{~K}$. The observed negative curvature of the $\ln 1 / \tau_{c}$ versus $1 / T$ relation is peduliar: It cannot be explained by is classical sum of activated processes, which 
would result in a positive curvature.

(ii) The activation enerey for $\mu^{+}$diffusion ( $150 \mathrm{meV}$, at least defined for the lower temperatures where an Arrhenius relation has been assumed) is smaller than for proton diffusion $(\sim 250 \mathrm{meV})$.

(iii) $\tau_{c}\left(\mu^{+}\right)>\tau_{p}$, although it seems that at the onset of diffusion the rates are of the same magnitude. In view of Eq. (1) this points to a considerably decreased mobility of the protons in the vicinity of the $\mu^{+}$and with respect to the $\mu^{+}$. Such a behaviour may indicate a high.ly correlated motion of protons and the $\mu^{+}$. It might also hint to the trapping of $\mu^{+}$in areas of largely immobile protons.

An alternative explanation could be the following: The contribution from the ${ }^{139} \mathrm{La}$ nuclei to the observed second moment cannot be ignored [2]. At low temperacure we have measured the muon spin relaxation in a metallic Lalli ${ }_{5}$ block. This allowed us to estimate a ${ }^{139}$. La contribution of about $0.06 \mathrm{\mu s}^{-1}$ to the $\sigma$ for the immobile $\mu^{+}$. Let us assume now that almost up to room temperature the $\mu^{+}$stays trapped in the lattice. This could be so if the muon either occupies one single location or has entered a rapid localized jumping mode as considered for the proton in 
Lall $i_{5} H_{6}$ by different authors $[11,12]$. The $\mu^{+}$then is less mobile because i.t requires a larger local lattice expansion due to its higher $z \in$ ro point energy compared to the proton. The motion of the protons would reduce a fraction of the field spread at the $\mu^{+}$ (averaging starting at $160 \mathrm{~K}$ and roughly completed at $250 \mathrm{~K})$, but the fraction due to ${ }^{139} \mathrm{La}$ would remain effective (at least partially in the case of localized jumping).

\section{3. $\underline{P d f} x$}

Our sample was a polycristalline Pd sphere (purity $5 \mathrm{~N}$, diameter $15 \mathrm{~mm}$ ) charged in a $\mathrm{H}_{2}$ atmosphere to the hydrogen content of $x=0.59,0.70$ and 0.75 . The higher concentrations of $x=0.81$ and 0.86 were reached by additional electrolytical charging; after preparation these samples were kept at liquid nitrogen temperature.

For the concentrations $x=0.70$ and 0.75 stroboscopic $\mu S R$ measurements [13] as well as zero field or transverse fleld time differential measurements were performed between $16 \mathrm{~K}$ and room temperature. At the three other concentrations similar measurements were effectuated for $\mathrm{T}<77 \mathrm{~K}$. 
Below $50 \mathrm{~K}$ a statio muon behaviour is indicated by the zero field measurements for all concentrations. Fri that temperature region fig. 3 displays the obtained static danping $\sigma$ versus hydrogen concentration (the reduced value $2 / 5 \times \sigma_{Z F}$ is shown for the zero field data). For $x>0.7$ a constant value $\sigma=0.124 \mathrm{\mu s}^{-1}$ is observed, compatible with the older measurements ry Fiory et al. at $x=0.97[14]$. The value of $\sigma$ is reduced for $x=0.70$ and 0.59 , but only by about $15 \%$ and $20 \%$, respectively.

Above $50 \mathrm{~K}$ the relaxation data for $\mathrm{x}=0.70$ and 0.75 were analysed using motional narrowing relations. The obtained correlation rates $i / \tau_{c}$ are presented in the conventional Arrhenius plot of fig. 4. The results obtained from the transverse field stroboscopic data and the zero field measurements are identical. They do not point to a striking hydrogen concentration dependence .

As it can be seen (Fig. 4), the correlation rate $1 / \tau_{c}$ is not described by a single Arrhenius law over the investigated temperature range. Above $100 \mathrm{~K}$ the rate follows an Arrhenius law with activation energy of $\sim 53 \mathrm{meV}$ and preexponential factor of about $3 \times 10^{7} \mu \mathrm{s}^{-1}$; below $100 \mathrm{~K}$ the data level off.

The diffusion of protons in $\beta-P d l$ has been studied by NMR [6-8] and neutron diffraction [9]; the reported activation energies $E_{a}$ scatter from $100 \mathrm{meV}$ to $250 \mathrm{meV}$. One golid line in Fis. 4 represents the results of I to et al [G], with $E_{a}=130 \mathrm{meV}$, which were oltained from a CW-NMR study 
in $\mathrm{PdEl} 0.75$ in the same temperature range as in our study. A second solid line shows the results of seymour et al. [7] for $x=0.70$ with $E_{a}=228 \mathrm{meV}$, and a third solid line links low temperature measurements by Kreitzman et al. [8] for $x=0.82$. Obviously the IMR and $\mu$ SR experiments yield quite different results. There is appreciable $\mu^{+}$diffusion for the low temperatures at which the protons are practically immobile. on the other side, above $180 \mathrm{~K}$, the $\mu^{\dagger}$ correlation rate is significantly reduced compared to the proton NMR results. If trapping is to be made responsible for this high temperature behaviour, one has difficulties to understand the low apparent activation energy. This might again be indicative of either a slow motion inside some region in which both the $\mu^{+}$and the protons are trapped or a highly correlated $\mu^{+}$-proton displacement.

The fact that $\sigma$ is constant for $T<50 \mathrm{~K}$ and $\mathrm{x}>0.7$ suggest that the $\mu^{+}$has an identical proton environment for this concentration range. 


\section{References}

[1] T.K. Halstead, J. Solid State Chem. 11 (1974) 114 .

[2] R.G. Earnes, W.G. Harper, S.O. Nelson, D.K. Thome and D.R. Torgescn, J. Less-Common Met. $49(1976) 483$.

[3] T.K. Halstead, N.A. Abood and K.H.J. Buschow, Solid State Comm. 19 (1976) 425 .

[4] R.C. Bowman Jr., D.M. Gruen, and M.H. Mendelschn, Solid State Comm. 32 (1979) 501 .

[5] D. Richter, R. Hempelmann and L.H. Vinhas, J. Less-Common Met. 88 (1982) 353.

[6] T. I to and T. Kadowaki, Phys. Lett. 54A (1975) 61 .

[7] E.F.W. Seymour, R.M. Cotts, and W.D. Williams, Phys. Rev. Lett. 35 (1975) 165.

[8] S.R. Kreitzman and R.I. Armstrong in Electronic Structure and Properties of Hydrogen in Metals, ed. P. Jena and C.B. Satterthwaite (P?enum, 1983).

[9] G. Nelin and K. Sköld, J. Phys. Chem. Sol. $\underline{36}$ (1975) 1175 . 
[10] F.H. Gygax, A. Hintermann, W. RueEg, A. Schenck, H. Studer, L. Schlapbach, and F. Stucki in Recent Developments in Condensed Matter Physics, ed. F.T. DeVreese et al., Vol. 2 (Plenum, 1981).

[11] J.C. Achard, A.J. Dianoux, C. Lartigue, A. Percheron-Guegan and F. Tasset j.n Rare Earth in Modern Science and Technology, Vol. 3 , ed. J. McCarthy and J.J. Rhyne (Plenum, 1981).

[12] D.G. Westlake in Electronic structure and Properties of Hydrogen in Metals, ed. P. Jena and C.B. Satterthwaite. (Plenum, 1983).

[13] F.N. Gygax, A. Hintermann, W. Ruegg, A. Schenck, W. Studer, A a J van der Wal and $L$. Schlapbach, Hyperfine Interactions, to be published,

F.N. Gygax, A. Hintermann, W. Ruegg, A. Schenck and W. Studer, Solid State Comm. 38 (1981) 1245 .

[14] A.T. Fiory, D.E. Murnick, R.P. Minnich and W.J. Kossler, Solid State Comm. 21 (1977) 747. 
Pigure Captions

Fig. 1 : Measured muon depolarization rate $\Lambda$ versus temperature in $\mathrm{LaNi}_{5} \mathrm{H}_{6} \cdot(1 / \Lambda$ is the time for which the polarization decreases to $1 / \mathrm{e}$ of its initial value.) The dotted level indicates the static Gaussian damping rate reached before onset of motional narrowing. The dashed curve represents the proton NMR line width [1] scaled down for comparison with $\mu^{+}$depolarization.

Fig. 2: Correlation rate $1 / \tau_{c}$ (logarithmic scale) versus inverse temperature for $\mu^{*}$ in LaN $i_{5} \mathrm{H}_{6}$. The straight solid line represents proton NMR results [3]. A data point from proton diffusion studied by quasielastic neutron scattering [5] is also indicated.

Fig. 3: Static Gaussian damping rate $\sigma$ versus hydrogen concentration $x$ measured for $\mu^{\dagger} S R$ in Palf $x$ below $50 \mathrm{~K}$. The different measurement techniques are indicated. (The reduced value $2 / 5 \times \sigma_{Z F}$ is represented for the zero field data.) The transverse field measurement by Fiory et al. [14] is also shown. 
Fig. 4: Correlation rate $1 / \tau_{c}$ (logarithmic scale) versus inverse temperature for $\mu^{+}$in $\beta-\mathrm{PdH}_{x}$. For comparison proton $11 M R$ results by $I$ to et al. [6], Seymour et al. [7] and Kreitzman et 1. [8] are also indicated. 


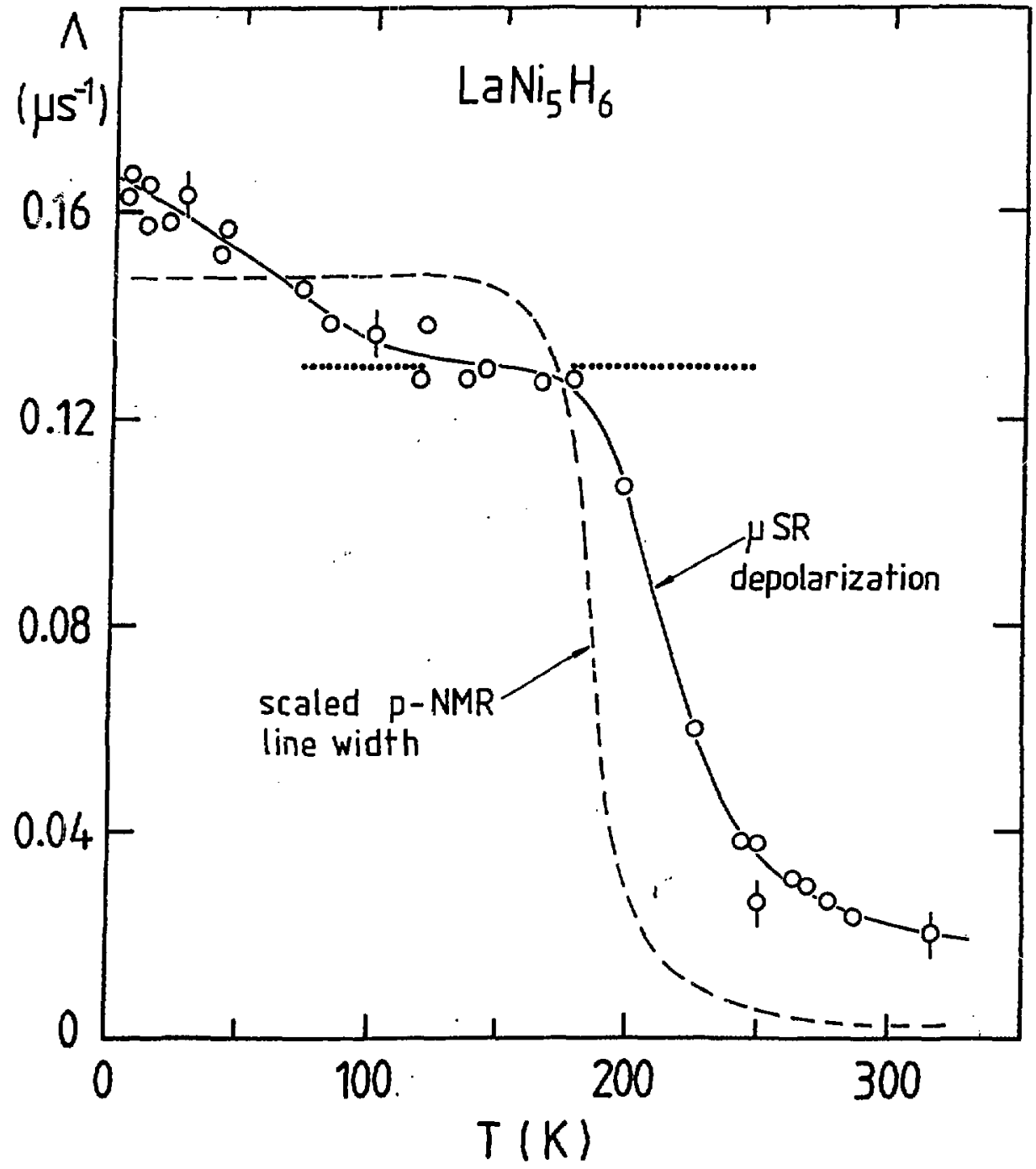

Figure 1 


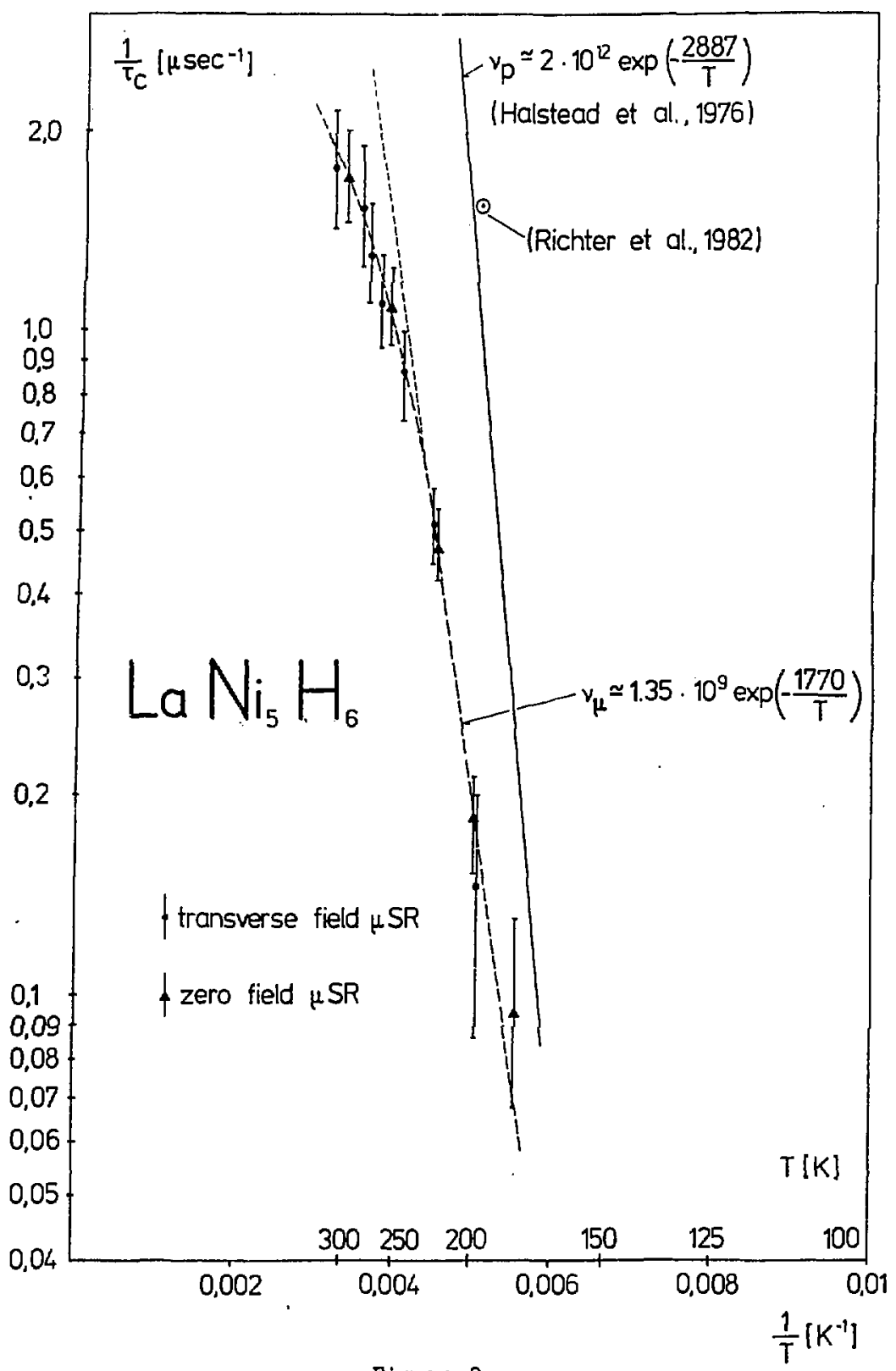

Figure 2 


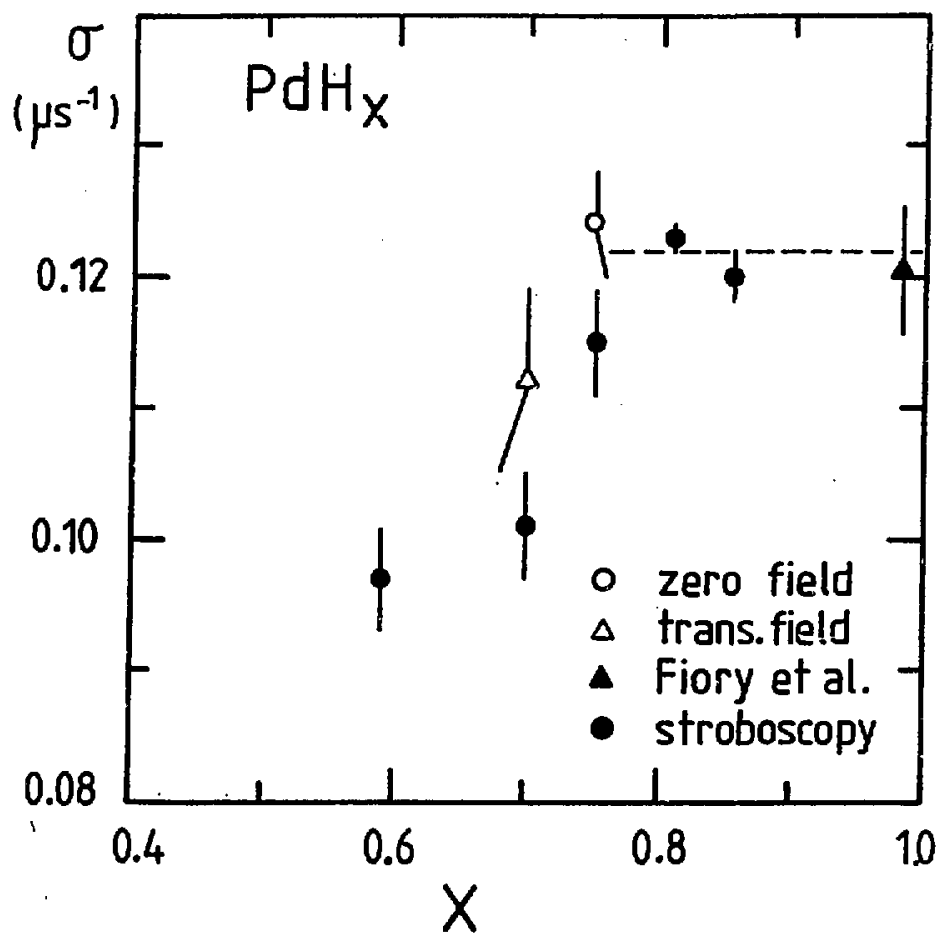

Figure 3 
- 1490

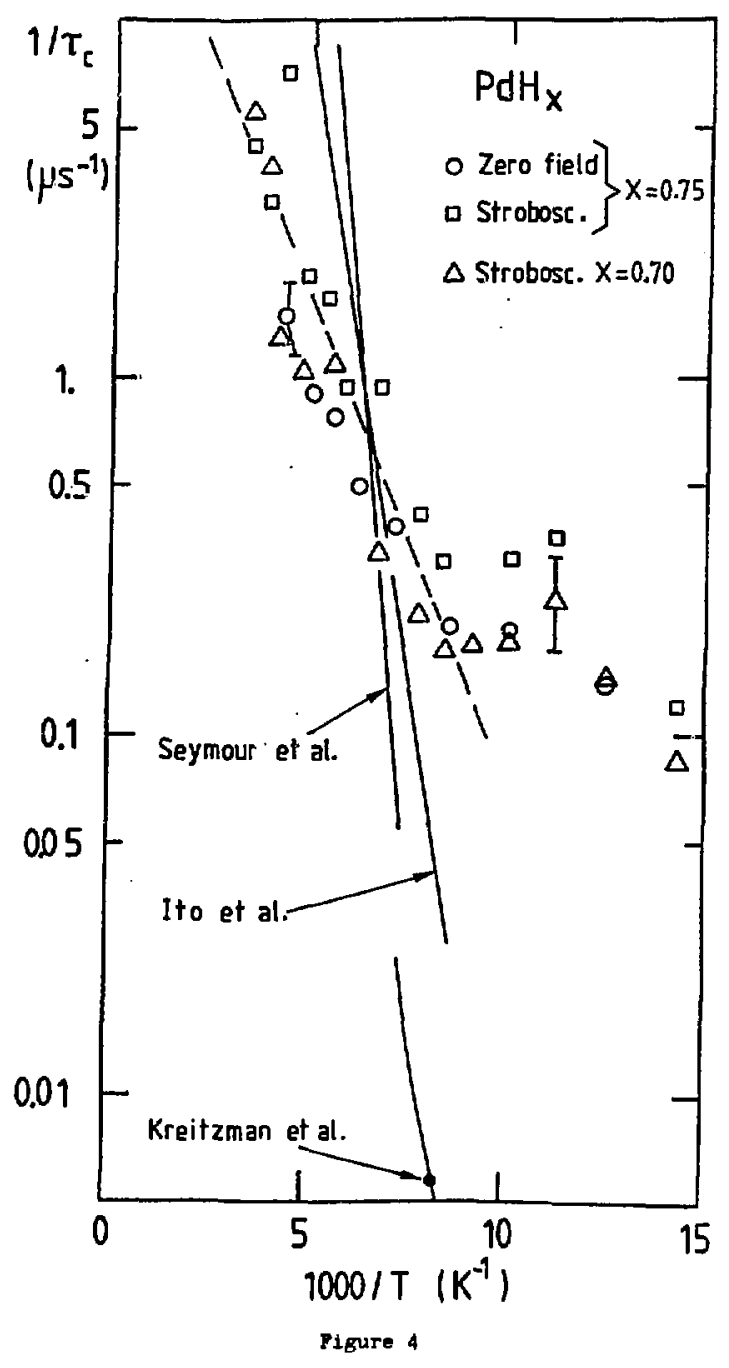


DIPFUEION AHD LOCALIZATION OF POSITIVE MUONE IJ HIORIUN GINGLE CRYSTALS AL LUW TEMPERATURES IH THE PRESENCE OF IMPURITIES MEASURED IN ZERO MND HIGH TRANSVERSE FIELD

F.II. Gygax *, A. II intermann *, N. Kaplan **, H. FuegE*, A. Schenck *, W. Studer * and A.J. van der WaI *

* Institute for Medium Energy Physics of ETII, c/o SIl, CH-5234 Villigen, Switzerland

* Racah Institute of Physics, Hebrew Iniversity of Jerusalem, Israel.

Presented as "Hydrogen in Metals" Conference, Wroclaw, September 1983

(to be published in J. Less Common Met.) 
ABSTRACT

We have studied the trapping and diffusion behavior of positive muons in Nb single crystals by comparing the measured depolarization rate with model calculations. Zero field $\mu \mathrm{SF}$ measurements reveal the temperature dependence of the diffusion coefficient and also clearly indicate that the different trap configurations above and below $\approx 22 \mathrm{~K}$ are correlated. The activation energy needed to escape from the low temperature traps was found to be $200 \pm 20 \mathrm{~K}(17 \pm 1.5 \mathrm{meV})$. Subseciliently, the angular dependence of the depolarization rate has been measured at 14.0 and $36.8 \mathrm{~K}$ in a transverse field of $7.47 \mathrm{kG}$ by rotating a $\mathrm{Nb}$ single crystal around its $\langle 110\rangle$ axis, which was kept perpendicular to both field and $\mu^{+}$polarization. This allows one to determine the site symmetry of the trapped muor as weIl as the lattice distortions around it. For $36.8 \mathrm{~K}$ the trappine site could be identified as a tetrahedral site next to a Ta impurity, possibly also close to an interstitial (fi or 0 ) impurity. A local lattice relaxation $\Delta R / A$ of $6.7(6)$ f for $n:$ and of $-6(2) \%$ for $n n n$ has been deduced. The low temperature curve shows a much more pronounced aneular dependence thar the high temperature curve, indicating a completely different $\mu$-environment. A satisfactory explanation for these date has not yet been found. 


\section{IITIRODUCTION}

In general, a close analogy exists belween the diffusion behavior of light interstitials in metals, such as hydrogen, and the positive muon, the main differences stenmine from the mass ratio of about ten. In contrast, the depolarization rate of positive muons implanted in relatively pure bec Nt vs. temperature shows intrigling aspects, not observed in the temperature dependence of the relaxation of hydrogen in this metal $[1,2]$. Among the fascinating features that puzzle both theoreticians and experimenters is the occurrence of a distinct dif at $\sim 22 \mathrm{~K}$. Although such a dip in the $\mu^{+}$-depolarization rate is not seen in other well-studied metals like e.g. Cu $[3,4]$, this feature is not unique. Similar structures have been observed in $B i, T a$ and $V$.

At low temperatures the depolarization rate in IIt shows two plateau regions separated by a more or less pronounced dip at $22 \mathrm{~K}$, depending on the concentration of residual impurities $[1,2,5,6]$. For temperatures higher then $\sim 50 \%$ the depolarization rate decreases to zero, as motional narrowing: sets in. In the case of ultrapure Nb, however, it was found $[7,3]$ that the $\mu \mu^{\dagger}$-depolarization rate is relativeiy ion, with hardy any structure from 5-50 K. Hartmann et al. [8] 2130 demonstrated that small amounts of impurities $(10-50$ ppm) such as Ta and it already suffice to create the dip, although it appears difficult to clearly separate their individual effects. 
EXPEIRIAENT AID DISCUSSION

I. Zero-field experiments

Two different theories have been forwarded to account for the observed behavior $[1,2,9]$. Petzinger [10] pointed out that zero-field experiments could distinguish between diffusion towards or escape from traps and therefore might discriminate between the two proposed descriptions.

Therefore, we measured the depolarization rate in a $\mathrm{Nb}$ single crystal of cylindrical shape with axis along $\langle 100\rangle$ in the region of the dip in zero field [11]. Fig. 1 shows the depolarization rate vs. temperature extracted from a series of zero-field measurements by fitting a Gaussian depolarization function. The same spectra have also been fitted with the dynamic Kubo-Toyabe (CKI) function [12] and the Petzinger function

$$
P(t)=\frac{1}{3}+\frac{2}{3} e^{-t / \tau_{c}}+\frac{2}{3} \int_{0}^{t} \frac{e^{-\tau / \tau_{c}}}{\tau_{c}}\left[1-\sigma^{2}(t-\tau)^{2} e^{-1 / 2 \sigma^{2}(t-r)^{2}}\right] d \tau
$$

where $\tau_{c}^{-1}$ represents the rate of a muon finding a trap. and $\sigma$ the static (Gaussian) relaxation rate.

If a mon escapes from a trap and moves around, it will finally depolarize completely as reflected by the DKT function. In contrast, a finite residual polarization will remain for $t \rightarrow \infty$, if the muon becomes trapped. 
From our results it is apparent that the petainger function fil:s the high temperature side of the dip $(22-28 \mathrm{~K})$ best, whereas the DKT function proved an excellent fit for temperatures in the range 14-22 K. In FiE. 2 , the resulting correlation rates $\tau_{c}^{-1}$ are plotted as a function of inverse temperature. The values for $\bar{C}_{c}^{-1}$ entered in Fig. 2 are obtained using static depolarization rates $\sigma$ of 0.515 and $0.500 \mathrm{MHz}$ for the DKT and Petzinger fits, respectively. Open circles represent the Petzinger fits and closed circles the DKT fits. A.lso inserted are three points (closed squares) representing $\tau_{c}^{-1}$ as derived from data taken on the same crystal at the low-temperature side of the dip in a transverse field of $200 \mathrm{G}$. These spectra have been fitted with the Abragam formula describing the motional averaging $[13]$.

Since $\tau_{c}^{-1}$ (Petzinger) is in the present dilution proportional to the concentration of availeble traps in the host, and $\tau_{c}^{-1}$ ( $D K T$ ) can be identified as the escape rate from the trap, i.e. an intrinsic trap property, the coincidence of these tyo rates both in slope and exponential prefactor in the same Arrhenius plot is quite striking. Although the equality of the exponential prefactors is probably fortuitious, the equality of both exponents at high-and low temperature side of the dip is clear evidence for the strong correlation of the mion motion above and below $21 \mathrm{~K}$. An explanation for this phenomenon could be that one observes here a bottleneck situation in which :11 muons that escape for $T>14 \mathrm{~F}$ from the low-temperature trap are quickly trapped by deeper, less abundant t, rapa. Whus the rate 
as which muons are finding the high-temperature traps is completely determined by the escape process. This in turn would imply that the two trap configurations at 14 and $35 \mathrm{~K}$, respectively would be situated relatively near to each other, though not as nearest neighbors. This last conclusion follows from the observation that the muon depolarization rate displays a clear dip, i.e. is clearly influenced by motional narrowing in going from one trap to the other.

In view of these observations and preceding the conclusions of the following section, we arrive at the following picture. At temperatures below $14 \mathrm{~K}$ the muon diffuses through the Nb host lattice forming a small polaron and thus finds the most abundant shallow trap near a Ta impurity, of which the exact configuration has not yet been resolved. An alternative interpretation would be that the muon is already trapped in the temperature range $5-14 \mathrm{~K}$. In that case the muon is assumed to be in a spatially extended state consisting of a number of equivalent sites, between which the muon quickly traps. With increasing temperature this state contracts onto a single site, as reflected by the slow increase in $\sigma$ with temperature. The fact that neither Petzinger nor DKT fits provide a satisfactory fit compared to static KT fits supports this interpretation.

At a temperature of $14 \mathrm{~K}$ the main fraction of muons is trapped at $\mathrm{I}$ a and as the temperature increases, muons start to move out of these traps, giving rise to motional narrowing and hence the occurence of the dip in the depolarization rate. Nt still higher temperatures $(\succsim 21 K)$ the muons find deeper, less 
abundant traps, which are connnected with both $\mathrm{Ta}$ and $\mathrm{N}$ (or $\mathrm{O}$ ) impurities, as will be discussed in the next section. It is known that interstitial impurities form deeper traps than substitutional [14].

Since our present samples contain 250 ppm Ta impurities and a total of about 100 ppm interstitial impurities $(0, N, C)$, the main fraction of muons, say 80\%, follows the hehavior outlined above. Of course, a remaining fraction traps directly at the $T a+N$ and thus does not participate in the formation of the dip. Once the muons are trapped in the $\mathrm{Ta}+\mathrm{N}$ trap complex, they only can escape via temperature activated hopping at $\gtrsim 80 \mathrm{~K}$. The connection of $\mathrm{Ta}$ with the plateau at $\sim 14 \mathrm{~K}$ and $\mathrm{N}$ (or 0 ) with high-temperature traps has already been discussed by other authors [8], a new element being introduced here is the hypothesis thet most of the interstitials trap near a Ta impurity both below and above the dip, as supported by the observed angular dependence of $\sigma$ in the following section. Of course, the preceding discussion does not exclude that some muons might move around in bandlike states, thus not contributing to $\sigma$ at all. 
II. High-field angular denendence at 14.0 and $36.8 \mathrm{~K}$.

In order to determine the sites at which the muon is trapped at both sides of the dip, the full angular dependence of the depolarization rate was measured by rotating a large single crystal around its $\langle 110\rangle$ cylinder axis in a transverse field of $7.47 \mathrm{kG}$. In a bcc lattice the muon is expected to reside either on tetrahedral sites located in the (001) planes or on octahedral sites located in the $\langle 100\rangle$ direction between two $\mathrm{Nb}$ sites. Theoretically, the depolarization rato can be calculated on the basis of the dipole-dipole interaction between the nuclear $N b$ moments $(I=9 / 2)$ and the muons spin $(I=1 / 2)$. The calculation yields the Gaussian depolarization rate in the so-called Van Vleck limit, viz. for magnetic fields high enough $(\geqslant 2 \mathrm{kG})$ to quench the quadrupole interaction between the $N \mathrm{~b}$ and the electrical field gradient induced by the positive muon.

In Fig. 3 the results are presented for the low-temperature plateau at $14.0 \mathrm{~K}$ (open circles) and for the high-temperature plateau at $36.8 \mathrm{~K}$ (closed circles). Also inserted is the result of a rigid lattice calculation in pure $\mathrm{Nb}$ for a tetrahedral site, appropriately averaged over all possible equivalent sites. As can immediately be seen from Fig. 3 , the absolute value of the experimental depolarization rate is much smaller than the calculated one for a tetrahedral site. For an octahedral site the difference would even be lasger. 
In order to explain the data for the high-temperature plateau, the solid curve was calculated under the assumption that the muon is trapped at a regular tetrahedral place, one of its nearest neighbors ( $n n$ ) being a Ta substitutional impurity, another $n n a \mathrm{Nb}$ atom shifted away from the $\mathrm{Ta}$, and two unrelaxed Nb $n n$. Only one of the next nearest neighbors (nnn) was shifted from its ideal position. After rearranging nn and nnn in this way, all nn's and nnn's are relaxed radially (as seen from the muon) to obtain a best fit to the measured data. Details on the exact positions of the nn's and nnn's can be found in Ref. 15 .

The fit resulted in a relative relaxation $\Delta R / R$ of $+6.7(6)$ for nn and $-(6 \pm 2) \%$ for nnn, where the minus sign refers to inward displacement. The $n n$ value is in reasonable agreement with the value of $5.4 \%$ obtained for the lattice relaxation of $\mathrm{Nb} \mathrm{nn}$ adjacent to interstitital $\mathrm{H}$ in $\alpha-\mathrm{Nb}[16]$. The displacements of nn's and nnn's that cause the resultant relaxation around the $\mu^{t}$ to be nonradial could, apart from the presence of the $T a$, be caused by an 0 or N interstitial located at the octahedral site between the $\mathrm{Ta} \mathrm{nn}$ and $\mathrm{a} \mathrm{Nb} \mathrm{nnn}$. The site assignment for the high temperature plateau and its possible connection with an interstitial impurity does not comply with the interpretation of Hartmann et al., [8], although their observations do not preclude the present site assignment of a combined Ta-N trap.

The analysis of the curve at $14 \mathrm{~K}$ presents more problems. Clearly the muon does not reside at an undistorted octahedral or tetrahedral site, a prime difficulty being the large difference in depolarization rate between $\langle 110\rangle$ of $\langle 111\rangle$ on one hand and 
$\langle 100\rangle$ on the other. The difference of about 30 is clearly not consistent with a tetrahedral site $(\approx 20 \%)$ nor with structures composed of such sites, e.g. the $T_{2}, T_{4}$, and $T_{6}$ tunnel states, proposed for $\mathrm{H}$ in $\mathrm{Nb}[17,18]$. These wil have an increasingly less pronounced angular dependence. Alternatively, an octahedral site assignment presents difficulties in accounting for the width of the peak around $H /\langle 100\rangle$ and the flatness of the curve around $H / /\langle 111\rangle$ and $\langle 110\rangle$.

Finally, one can consider the influence of the finite extension of the muon wave function in the host matrix on the second moment and thus on the $\mu^{+}$-depolarization rate. This problem is treated theoretically by various authors $[19,20]$. From the work of Meier [21] one knows that as long as the $\mu^{t}$ wavefunction is spherically symmetric, the dipolar field and, by the same arguments, the second moment due to dipole-dipole interaction are not changed by the finite spatial extension. Following the work of Mcllullen et al. and inserting the values for the width of the $\mu^{+}$wevefunction, approximated by a nonisotropic Gaussian function, as given by Sugimoto et al. for $\mathrm{Nb}$, one calculates a reduction of the second moment of only a few percent compared to a pointlike muon. For the present discussion we can therefore safely ignore the finite extension of the muon wavefunction.

In conclusion, we have been $a b=$ to reproduce the high-temperature angular dependence of the depolarization rate $\sigma$ with a possible trap configuration for the $\mu^{+}$in the $\mathrm{Nb}$ host lattice. Substitutional Ta impurities, possibly in combination with interatitial impurities like $O$ or $N$, play an 
essential rate in explaining the observed angular dependence of $\sigma$ in high magnetic field, together with an additional substantial radial outward relaxation of the neighbors nearest to the $\mu^{+}$. Moreover, zero-field data show that the low- and high-temperature plateaus around the dip at $\sim 22 \mathrm{~K}$ are strongly correlated, thus indicating that the majority of mons escaping from low-temperature traps find after several jumps deeper, less abundant traps. This observation is an indication that only one - single fraction of muons plays a dominant role in the detrapping-trapping behavio: around the dip, in contrast with mechanisms as discussed in Ref. 8 . Full analysis of the angular dependence at $14 \mathrm{~K}$ is in progress in order to resolve the trap configuration at low temperatures and further experiments have been planned to vary the concentration of interstitial impurities in the same single crystal and repeat the angular dependence measurements around the dip. 
FIGURE CAPTIONS

Fig. 1: Temperature dependence of the Gaussian depolarization rate $\sigma$ of positive muons implanted in a cylindrical $\mathrm{Nb}$ single crystal with axis along $\langle 100\rangle$. In a zero field experiment. The data-points represented by closed circles were taken about one year later than those represented by open circles.

Fig. 2: Arrhenius plot of the inverse correlation time $\tau_{c}^{-1}$ vs. inverse temperature. The open circles correspond to Petzinger fits and the closed circles to dynamic Kubo-Toyabe fits. Also inserted are three points (open squares) representing $\tau_{c}^{-1}$ as derived from data taken on the same crystal at the low-temperature side of the dip in a transverse field of $N 200 \mathrm{G}$ and fitted with the Abragam formula pertaining to motional narrowing. The solid line is a least-squares fit to $\tau_{C}^{-1}=A \exp \left(-E / k_{B} T\right)$, resulting in an 'attempt rate" $A=28 \pm 4 \mathrm{GHz}$ and an activation energy $\mathrm{E} / \mathrm{k}=200 \pm 20 \mathrm{~K}$. The $\tau_{c}$ 's derived from Petzinger and Kubo-Toyabe fits represent of course quite different quantities. Further discussion is deferred to the text.

Fig. 3: Angular dependence of the $\mu^{+}$-depolarization rate of a muon in $\mathrm{Nb}$ for the two plateau temperatures, $36.8 \mathrm{~K}$ (closed circles) and $14.0 \mathrm{~K}$ (open circles), 
respectively, corresponding to high and low temperature trapping sites at both sides of the dip. All measurements were done in a field of $7.47 \mathrm{~kg}$ with the stroboscopic $\mu \mathrm{SR}$ technique. The solid line is the result of a second moment calculation allowing for nonradial relaxation around the muon of nearest neighbors and of next-nearest neighbors, due to the presence of substitutional $(\mathrm{Ta}$ ) and interstitial (N) impurities and the $\mu^{+}$itself. For reference, the theoretical angular dependence of a tetrahedral site in a pure $\mathrm{Nb}$ lattice without any relaxation is also shown. 
[1] H.K. Birnbaum, M. Camen1, A.T. Fiory, F.N. Gygax, W.J. Kossler, W. Rllegg, A. Schenck, and H. Schilling, Phys. Rev. B17 (1978) 4143.

[2] M. Borghini, T.0. Niinikoski, J.C. Soulié, 0. Hartmann, E. Kerlsson, L.O. Norlin, K. Pernestal, K.W. Kehr, D. Richter, and E. Walter, Phys. Rev. Lett. 40 (1978) 1723 .

[3] M. Camani, F.N. Gygax, W. Rüegg, A. Schenck, and H. Schilling, Phys. Rev. Lett. 39 (1977) 836.

[4] H. Schilling, M. Camani, F.N. Gygax, W. Rüegg, and A. Schenck, Journ. Phys. F12 (1982) 875 .

[5] J.A. Brown et al., Hyperfine Interactions $\underline{6}$ (1979) 233.

[6] R.H. Heffner, J.A. Brown, R.I. Hutson, M. Leon, D.M. Parkin, M.E. Schillaci, W.B. Gauster, O.N. Carison, D.K. Rehbein, and A.T. Fiory, Hyperfine Interactions $\underline{6}$ (1979) 237 .

[7] T.0. Niintkoski, 0. Hartmenn, E. Karlsson, L.-0. Norlin, K. Pernestll, K.W. Kehr, D. Richter, F. Walker, and K. Schulze, Hyperfine Interactions $\underline{6}$ (1979) 229.

[8] 0. Ilartmann, E. Karlsson, R. Waeppling, D. Richter, R. Hempelmann, K. Schulze, B. Patterson, E. Ilolzschuh, W. Kuendig, and S.F.J. Cox, Phys. Rev. B27 (1983) 1943. 
[9] V.G. Grebinnik, I.I. Gurevich, V.A. Zhukov, A. Klimov, V.N. Maiorov, A.P. Manych. E.V. Mel'nikov, B.A. Nikol'skii, A.V. Pirogov, A.N. Ponomarev, V.I. Selivanov, and V.A. Suetin, JETP Lett. 25 (1977) $29 \hat{8}$.

[10] K.G. Petzinger, Phys. Lett. $\underline{75 A}$ (1980) 225 and K.G. Petzinger, Hyperfine Interactions 8 (1981) 639.

[11] A preliminary account of these zero-field measurements has been published as F.N. Gygax, A. Hintermann, N. Kaplan, W. Rulegg, A. Schenck, W. Studer, and A.J. van der Wal, SIN-Newsletter no. 14, 1982. Similar measurements are reported by C. Boekema, R.H. Heffner, R.I. Hutson, M. Leon, M.E. Schillaci, W.J Kossler, M. Numan, and S.A. Dodds, Phys. Rev. B26 (1982) 2341.

[12] R. Kubo and T. Toyabe: In Magnetic Resonance and Relaxation, ed. by R. Bline (North Holland, Amsteraam, 1967).

[13] See e.g., A. Abragam: Principles of Nuclear Magnetism (Clarendon Press, Oxford, 1970).

[14] See e.g. Ch.A. Wert: In Hydrogen in Matals II, ed. by G. Aleveld and J. Voelkl (Springer, Berlin, 1978) and Ref. 20. 
[15] F.N. Gygax, A. Hintermann, W. Rülegg, A. Schenck, W. Studer, A.J. van der Wal, and N. Kaplan, Proceedings Yamada Conference on $\mu \mathrm{SR}$, Shimoda 1983, to be published in Hyperfine Interactions.

[16] H. Behr, H.M. Keppler, G. Steyrer, H. Metzger, and J. Peisl, J. Phys. F13 (1983) L29.

[17] W.F. Lankford, H.K. Birnbaum, A.T.Fiory, R.P. Minnich, K.G. Lynn, C.E. Stronach, I:H. Bieman, W.J. Kossler, and J. Lindemuth, Hyperfine Interactions 4 (1978) 833.

[18] H. Wipf, A. Magerl, S.M. Shapiro, S.K. Satija, and W. Thomlinson, Phys. Rev. Letters $\underline{46}$ (1981) 947.

[19] T. MoMullen and E. Zaremba, Phys. Rev. B18 (1978) 3026.

[20] H. Sugimoto and Y. Fukai, Phjs. Rev. B22 (1980) 670.

[21] P.F. Meier: In Exotic Atoms 1979, ed. by K. Crowe, J. Duclos, G. Fiorentini and G. Torelli (Plenum, N.Y.). 


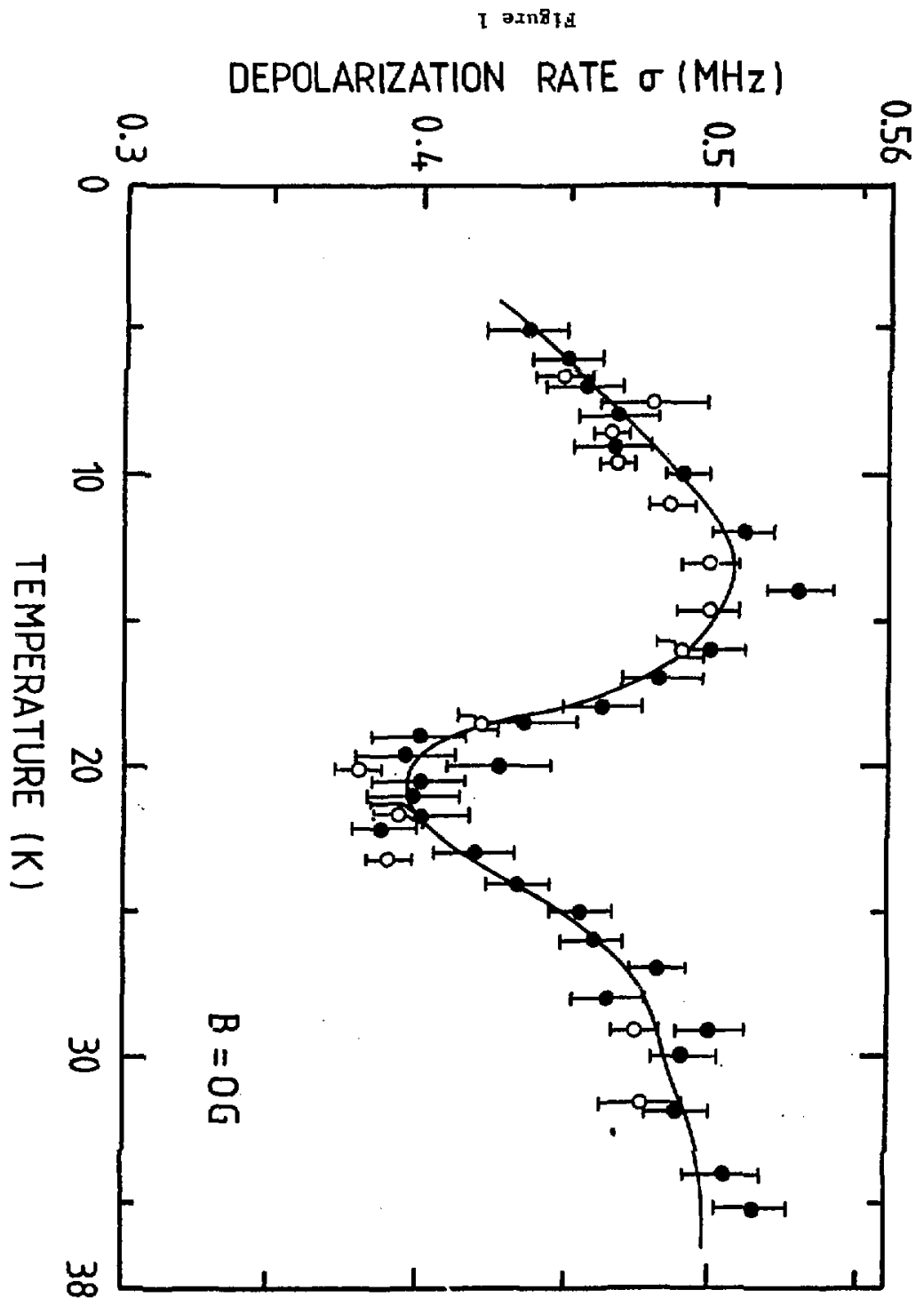




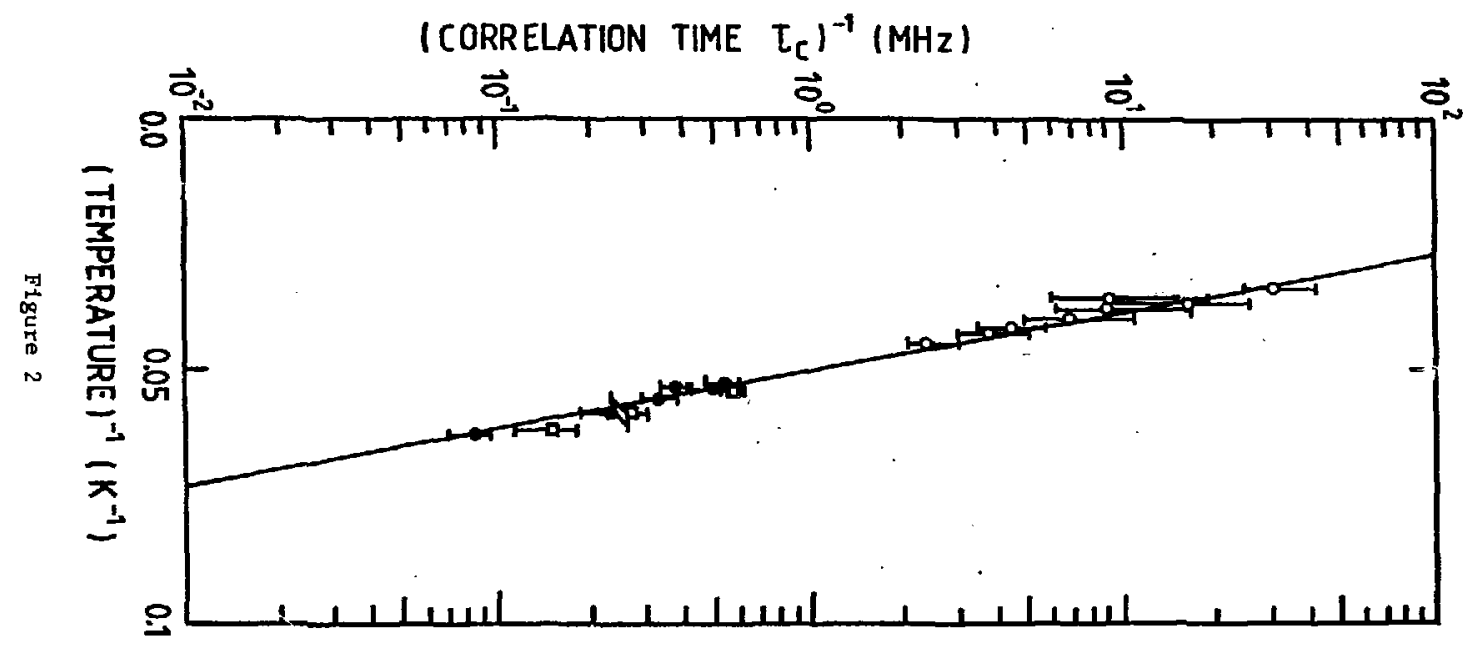

봉 


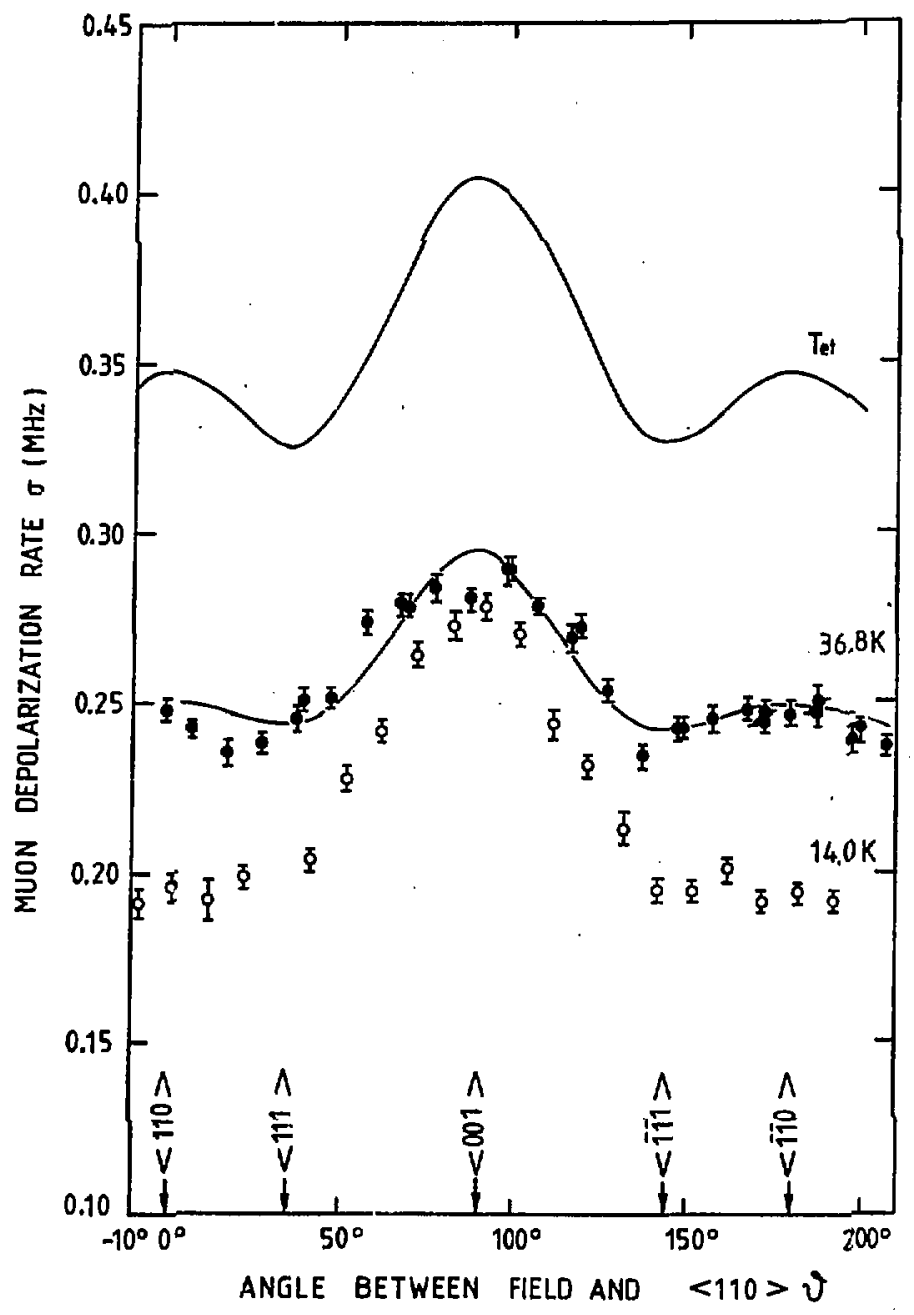

Pigure 3 
$\mu^{+}$-KNIGHTSHIFT STUOIES AND THE ELECTRONIC STRUCTURE OF HYOROGEN IN METALS

F.N. Gygax, A. Hintermann, W. Rüegg, A. Schenck, W. Studer and $A$. van der Wal

Institute for Medium Energy Physics, ETH Zurich, c/O SIN, $\mathrm{CH}-5234$ Villigen, Switzerland

1

Abstract

An up to data review of positive muon $\left[\mu^{+}\right]$Knight shift studies in elemental non transition and transition metals and in B-palladium hydride will be presented. Implisations on the local electronic structure of a hydrogenlike impurity will be pointed out.

Invited paper at International Meeting on Hydrogen in Metals (Wroclaw, Septomber 1983)

(to be published in J. Less Common Met.) 


\section{Introduction}

Interstitial protons or positive muons $\left(\mu^{+}\right)$in a metal crystal aquire nearly the same electron distribution in their vicinity due to their identical charge state and strong Coulomb potential. Possible differences stem from their different zero point vibration amplitudes $\left(m_{\mu} \approx 1 / 8 m_{p}\right)$ which brings them differently close to the host lattice constituents. Aside from such effects there is basically no difference between the electronic properties of the two particles in a metal environment. Information gained on the electronic structure around $a^{+}$can therefore be viewed as representative of the corresponding electronic structure around a proton. In this respect the $\mu^{+}$serves as a substitute probe in the study of the electronic structure of hydrogen in metals.

Pusitive muons can be implanted in any metal or metal alloy and its Knight shift can be measured by the muon spin precession ( $\mu S R$ ) technique with high precision. In contrast the proton Knight shift can only be measured in metallic systems which absorb sufficient quantities of hydrogen to allow application of the NMR technique [1]. 


\section{Knight shift and electronic structure}

The implanted $\mu^{+}$is surrounded by electrons which form the local electronic structure and screen the positive muon charge within a radius of typically $2 \cdot{ }_{B}$ [2]. The $\mu^{+}$and the electrons interact magnetically with each other according to the Hamiltanian (in the nonrelativistic Pauli approximation)

$$
\begin{aligned}
\mathscr{H} & =g_{\mu} \mu_{\mu} g_{e} \mu_{B} \vec{I}_{\mu} \cdot \varepsilon_{i}\left\{\frac{\vec{l}_{i}}{r_{i}^{3}}+\left[-\frac{\vec{s}_{i}}{r_{i}^{3}}+\frac{3 \vec{r}_{i}\left(\vec{s}_{i} \cdot \vec{r}_{i}\right)}{r_{i}^{5}}\right]+\frac{\theta \pi}{3} \delta(\vec{r}=0) \overrightarrow{s_{i}}\right\} \\
& =g_{\mu} \mu_{\mu} \vec{I}_{\mu} \cdot \vec{H}_{\text {eff }}
\end{aligned}
$$

where the $\mu^{+}$is located at $\vec{r}_{\mu}=0$. The index $i$ refers to the $i^{\text {th }}$ electron. The first term under the sum is the orbital, the secand term the dipole-dipole and the third term the Fermi cantact hyperfine interaction. The sum over the first term vanishes since no net angular momentum is formed at the $\mu^{+}$. However, in the presence of an external field the induced orbital motion of the electrons will lead to a diamagnetic screening field at the $\mu^{+}$(see below).

Introducing the charge density distribution

$$
\pi(\vec{r})=\sum_{i}\left|\Psi_{i}(\vec{r})\right|^{2}
$$


an effective magnetic field $\vec{H}_{\text {eff }}$ can be expressed in integral form as

$$
\begin{aligned}
\vec{H}_{g f f} & =g_{e} \mu_{B} \int_{V_{L}} n(\Gamma)\left[-\frac{\vec{S}(\vec{r})}{r^{3}}+\frac{3 \vec{I}(\vec{S}(\vec{\Gamma}) \cdot \vec{I})}{\Gamma^{5}}\right] d v \\
& +\frac{8 \pi}{3} g_{E} \mu_{B} n(0) \vec{S}
\end{aligned}
$$

The integration extends in principle over the total volume of the sample, but we will assume the probe to be of spherical shape so that the demagnetization field and the Lorentz field cancel and only the integration over the Lorentz sphere remains. In the absence of magnetic ordering or an external field $\vec{H}_{\text {eff }}$ will of course be zero. In the presence of an external field $\theta_{0}$ in, e.g. z-direction, each electronic spin will on the average acquire a small static component in $z-d i-$ rection which is given by

$$
\left\langle S_{z}>=X_{e} \cdot B_{o} \frac{1}{g_{e} \mu_{B}}\right.
$$

where $x_{e}$ is the spin susceptibility per electron (assumed to be independent of position $\vec{r}$ ). As a result we find a nonvanishing $H_{\text {eff }}$ in z-direction. The total field acting on the $\mu^{+}$is then given by 


$$
B_{\text {tot }}\left(\vec{r}_{\mu}=0\right)=B_{0}+H_{e f f}=B_{0}(1+K)
$$

where $K=H_{\text {eff }} / \theta_{0}$ is the so called Knight shift constant $K S$. and $H_{\text {eff }}$ the field shift. With Eq. (3) $K$ can be written as

$$
\begin{aligned}
K & =\frac{8 \pi}{3} n(0) x_{e}+\left[\int_{V_{L}} n(\vec{r}) \frac{3 \cos ^{2} \theta-1}{r^{3}} d v\right] x_{e} \\
& =K_{i s o}+K_{a x}
\end{aligned}
$$

where $\theta$ is the angle between the $z$-axis and $\vec{r}$. The second term of Eq. (6), the axial KS $K_{a x}$, vanishes for a charge distribution of spherical and cubic symmetry. For a charge distribution of axial symmetry $k_{a x}$ can be written as

$k_{a x}=\left[\int_{V} n\left(\vec{r}^{\prime}\right) \frac{3 z^{\prime 2}-1}{r^{13}} d v^{\prime}\right]\left(3 \cos ^{2} \theta-1\right) x_{e}$

where $x^{\prime}, y^{\prime}, z^{\prime}$ refer to a new coordinate system wita $z^{\prime}$ the axis of axial symmetry, usually to be identified with a certain crystal axis. $\theta$ is the angle between the $z^{\prime}$-axis and the external field or the z-axis, respectively. A nonspherical or noncubic charge distribution, therefore, leads to an anisotropic KS. However, also the first term $K_{i s o}$, stemming from the isotropic contact interaction may become anisotropic if the spin susceptibility is anisotropic. 
In simple, nontransition metals only $(s, p)$-electrons at the Fermi energy will become spinpolarized in the presence of an external field (Pauli spin paramagnetism), i.e.

$$
n(0)=n(0)_{F}=\left\langle|\psi(0)|^{2}\right\rangle_{F}
$$

and principally $K_{i s o}=K_{5}>0$. The effect of core polarization may alter $K_{i s o}$ in both directions.

To include this effect $K_{i s o}=K_{s}$ is often expressed in an obvious manner as

$$
\begin{aligned}
K_{S} & =\frac{8 \pi}{3} \mu_{B} \frac{n(0)^{\dagger}-n^{\dagger}(0)}{n_{0}^{\dagger}-n_{0}^{\dagger}}\left(n_{0}^{\dagger}-n_{0}^{+}\right) / B_{0} \\
& =\frac{8 \pi}{3} \frac{n^{\dagger}(0)-n^{\dagger}(0)}{n_{0}^{\dagger}-n_{0}^{\dagger}} x_{s}=\frac{8 \pi}{3} \rho_{s}(0) x_{s}
\end{aligned}
$$

where $\rho_{s}(0)$ is the so called spin density enhancement factor and $x_{5}$ is the valume spin susceptibility of the homogeneous electron gas characterizing the conduction electrons.

In d-electron and f-electron transition metals, where the $d$ and f-electrons are contributing dominantly to the magnatic susceptibility, core polarization must be very important since the direct overlap of d-electron and more so of $f-$ electron wave funcioions with the $\mu^{+}$is probably very small. We introduce therefore an additional term 


$$
K_{c p}=\frac{1}{\mu_{\theta}} B_{h f}^{\Omega a} x_{a t}(d, f)
$$

where $B_{h f}^{\text {Ra }}$ is the induced hyperfine field at the $\mu^{+}$per unpaired d-, f-electron per atom. $x_{\text {at }}$ is the atomis spin susceptibility of the $d-, f$-electrons. In many cases $x(d, f)$ shows a strong temperature dependence which allows to distinguish between $K_{c_{p}}$ and $K_{s}$. Core polarization could in principle also contribute to the axial KS.

A last contribution to a field shift is the already mentioned diamagnetic screening analogous to the chemical shift in atoms and molecules. This negative shift constant is given by the Lamb expression

$$
\sigma=-\frac{\mathrm{e}}{3 \mathrm{mc}^{2}}\left\langle 0\left|\frac{1}{\mathrm{r}}\right| 0\right\rangle
$$

which yields $\sigma \approx-20$ ppm rather independent of the host metal and close to the atomic hydrogen value of - $18 \mathrm{ppm}$ [3]. It involves the total charge distribution around the impurity in contrest to the contact term induced shift which depends only on the local densities at the impurity. The total field shift (usually also called Knight shift) is then in principle composed of the following terms

$$
k_{\mu}=k_{s}+k_{c p}+k_{a x}+\sigma
$$




\section{Knight shift in nontransition metals}

\section{1. Systematics_of _isotropic_Knight_shift}

Most of the data have already been discussed elsewhere [4, 5]. An updated compilation of all data is presented in Tab. I. New entries are the values of $K_{\mu}$ in $A u, Z n$ and $C d$. Revised values can be found for Be and $\mathrm{Mg}$, stemming from new measurements on high purity single crystal samples. For completeness Tab. I contains also results obtained in the semi metals Sb and $\mathrm{Bi}$, but these results will not be discussed here.

It was found earlier [4] that the logarithm of the iuduced hyperfine field per unpaired electron per atom, $B_{h f}^{\text {Sa }}=\mu_{B} K_{s} / X_{a t}(s) *$, showed a universal behavior in the cubic mono-, di- and tri-valent metals when plotted versus the molar electronic specific heat, which is proportional to the density of states at the Fermi energy (Fig. 2). It is now found that also the new values in $A u$ and in the hop metals $B e, Z n$ and $C d$ are consistent with this universal curve. Also the revised value in Mg is now closer to this universal curve. One consequence of this universal behavior was the abservation that the $\mu^{+}$KS shows a distinctive dependence on the valency of the metal and only to a lesser degree on the total conduction electron concentration in contrast to pre* In calculating $B_{\text {ha }}^{\Omega a}$ experimental values for $x_{\text {at }}$ were used
throughout. 
Table I

Compilation of $\mu^{+}-$Knight shift data in nontransition metals. $x_{S}=$ experimental spin susceptibility (from Refs. 4, 15).

\begin{tabular}{|c|c|c|c|c|c|}
\hline metal & $r_{s}$ & $x_{s}\left|\frac{\mathrm{emu}}{\mathrm{cm}^{3}}\right|$ & $K_{i s o}[\mathrm{ppm}]$ & $\mathrm{K}_{\mathrm{ax}}[\mathrm{ppm}]$ & Refs. \\
\hline & & $\times 10^{-6}$ & & & \\
\hline Li & 3.25 & 2.10 & $8.6 \pm 4.0$ & & 4,6 \\
\hline $\mathrm{Na}$ & 3.93 & 1.09 & $76.5 \pm 5.0$ & & 4,6 \\
\hline $\mathrm{K}$ & 4.86 & 0.90 & $63.2 \pm 4.5$ & & 4,6 \\
\hline $\mathrm{Rb}$ & 5.20 & 0.86 & $66.1 \pm 4.3$ & & 4,6 \\
\hline Cs & 5.63 & 0.81 & $31.0 \pm 4.0$ & & 4,6 \\
\hline $\mathrm{Cu}$ & 2.67 & 1.36 & $60 \pm 2.5$ & & 4,6 \\
\hline $\mathrm{Ag}$ & 3.02 & 0.88 & $94 \pm 3.5$ & & 4 \\
\hline $\mathrm{Au}$ & 3.01 & 0.99 & $64 \pm 10$ & & 7 \\
\hline $\mathrm{Be}$ & 1.88 & $\sim 0.2$ & $-9 \pm 1.6$ & $-3 \pm 1.9$ & 8,9 \\
\hline $\mathrm{Mg}$ & 2.65 & $\sim 1.58$ & $43.3 \pm 3.5$ & 0. & new value \\
\hline $\mathrm{Ca}$ & 3.27 & -1.58 & $21.2 \pm 6.2$ & & 4,6 \\
\hline $\mathrm{sr}$ & 3.56 & 22.69 & $-18.4 \pm 6.8$ & & 4,6 \\
\hline Ba & 3.69 & -0.99 & $29.6 \pm 5.0$ & & 4,6 \\
\hline $\mathrm{Zn}$ & 2.31 & $\sim 0.99$ & $60 \pm 5^{*}$ & $3 \pm 1.3^{*}$ & $8,9,10$ \\
\hline Cd & 2.59 & $\sim 0.73$ & $55 \pm 5^{*}$ & $-3 \cdot 3 \pm 1.3^{*}$ & $8,9,11$ \\
\hline $\mathrm{Hg}$ & 2.75 & $\sim 1.73$ & $117 \pm 11^{* *}$ & & 4 \\
\hline Al & 2.07 & 1.77 & $79.6 \pm 4.0$ & & 4 \\
\hline Ga & 2.19 & $\sim 0.7$ & $-17 \pm 3.5$ & & 4 \\
\hline $\mathrm{Pb}$ & 2.30 & -2.36 & $105.2 \pm 3.5$ & & 4 \\
\hline $\mathrm{Sb}$ & & $\sim 0,082$ & $8700^{* * *}$ & $2200^{* * *}$ & 12,13 \\
\hline Bi & & $\sim 0.014$ & $-276 * * * *$ & $+9 \pm 6 * * * *$ & new value \\
\hline
\end{tabular}

* $20 \mathrm{~K}, *-50^{\circ} \mathrm{C}, * * * 15 \mathrm{~K}, * * * 4.2 \mathrm{~K}$ (preliminary data), all other data are at roomtemp. 
dictions by various jellium calculations on the basis of the spin density functional forma?ism [2].

In the meantime considerable progress has been achieved in the understanding of the gross features of the observed systematics by the work of Manninen [14]. It is essentially also a jellium calculation, but the host lattice potential is taken into account by the so-called spherical solid model, which replaces the compensating positive background charge in the conventional jellium approach.

In this model the host potential is constructed from the unscreened pseudopotentials of the host ions and subsequently a spherical average is calculated around the $\mu^{+}$-site. This spherical average together with the $\mu^{+}$potential itself is then used as the relevant potential in a jellium calculation using again the selfconsistent spin density functional formalism, which includes automatically the effect of core polarization. In contrast to the conventional jellium calculations the actual lattice site of the $\mu^{+}$is now important and leads to different results. For the pseudo potentials the simple Ashcraft empty-core potentials with commonly used core radii were taken [14]. Other parameters that enter the calculations are the electron derisity parameter $r_{5}$ and the valency 2. As an example, Fig. 2 shows a partial-wave decomposition of the electron density at the tetrahedral $\mu^{+}$site 
in Cs obtained by this model. Also shown are the results of a nonpolarized jellium calculation. Clearly bound states show up showing a larger density for the spin down configuration which contribute therefore a negative hyperfine field. The continuum states, on the other side, contribute a positive hyperfine field. Compared with the jellium model the continuum states provide a considerably smaller contact density while the bound states provide a larger one, however both models yield almost the same total electron density at the $\mu^{+}$-site. In the end the model is used to calculate the spin density enhancement factor $\rho_{s}(0)$ introduced in Eq. (B). Fig. 3 and Fig. 4 show the predictions of the spherical solid model for $K_{\mu}$ in mono and divalent metals. The diamagnetic cantribution was calculated in the same model using a formula derived in [3]. The spin susceptibility, needed to calculate $\mathrm{K}_{\mathrm{g}}$. was taken to be the one of a homogeneous interacting electron gas of the appropriate density rather than the experimental value which is justified on grounds of internal consistency in using Eq. (8). It is evident from Figs. 3 and 4 that the general trend of the data with the density parameter $r_{s}$ is reproduced quite well as is the grouping of the data with the valency of the host metal. However, if experimental values for the spin susceptibility were used, we would have found less agreement. This is somewhat surprising in view of the striking systematics in Fig. 1 which was obtained by using experimental values. 
The situation is more clearly shown in Fig. 5 where we compare theoretical $\rho_{s}(0)$ from Manninen [14] with empirical ones calculated from the data as follows: $\rho_{s}(0)=\left(K_{\mu}-\sigma\right) / \frac{8 \pi}{3} x_{5}$. where $x_{S}$ are expe:imental values $[4,15]$ and the diamagnetic screening constant is taken to be -20 ppm for all samples. We notice that the deviations from the predictions are mast pronounced for the high density metals, although the trends are still in qualitative agreement.

Manninen [14] has also found an approximate linear relationship between $\log B_{h f}^{\Omega a}$ and the atomic density of states lassuming a homogeneous electron gas picture). He dismisses his resu?t as somewhat accidental. However in view of the empirical universal behavior in Fig. 1, which indicates that the true density of states matters indeed, we feel that it is neccessary to persue this problem further thaoretically and that the present theory, although successful in reproducing the gross feature of the data, still lacks a predictive power in detail. For instance there is quite a gap between the experimental and predicted $k_{\mu}$ in $C u$, while the experimental $B_{h f}^{\Omega a}$ is excellently accomodated by the universal curve in Fig. 1 . 


\subsection{Axial Knight_shift}

Careful measurements of the angular dependence of the $\mu^{+}-k S$ in single crystals of the hop metals Be, Cd and $\mathrm{Zn}[8,9]$ showed the clear presence of an axial KS, while no axial KS could be observed in $\mathrm{Mg}_{\mathrm{g}}$. The axis of axial symmetry coincided generally with the crystalline c-axis. Fig. 6 shows the results obtained in Be at room temperature. In $\mathrm{Zn}$ an anisotropic KS could only be observed at low temperatures ( $<$ BO K). Its size and functional dependence on angle varied between different samples and seemed to depend on the sample histary, like the speed of temperature changes. At present we cannot offer any interpretation of the Zn-data. In Cd an axial KS was abservable at all temperatures practically up to the melting point, however, a change of sign of $k$ ax occurred at $110 \mathrm{~K}$. Fig. 7 shows the angular dependence of $K_{\mu}$ at $60 \mathrm{~K}$ and at room temperature[18]. The peculiar temperature of $110 \mathrm{~K}$ shows also up in the temperature dependence of the isotropic KS (see next section). If the axial KS in Cd is due to a nonspherical charge distribution around the $\mu^{+}$, i.e. due to the presence of p-like states we would have observed a change of the charge distribution from oblate like ( $P_{x}$, $P_{y}$ components dominant) at low temperatures to prolate like ( $p_{z}$ component dominant) at high temperatures. On the other hand the observed anisotropy may be also due to an anisotrapic $x_{5}$ as a

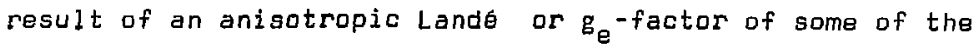


electrons contributing to the contact density. This is not an unreasonable possibility since it is known that spin-orbit coupling effects play an important role in the Cd band structure.

Since spin orbit coupling is of negligible importance in the low Z-metal be we conclude that the observed axial $\mu^{+} \mathrm{KS}$ there prosents the first evidence that also non s-like lwith respect to the $\mu^{+}$) electrons can be important in the local electronic structure of a hydrogenlike impurity.

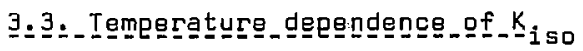

In nontransition metals one does not expect to find a significant temperature dependence, since the Pauli spin susceptibil. $\checkmark$ is temperature independent as long as one deals with a degenerate elertron system.

Inderd the $\mu^{+} \mathrm{KS}$ in $\mathrm{Cu}$ and $\mathrm{Al}$ does not display a temperature dependence within the present accuracy. In $Z n$ one finds a slight linear increase with temperature with a slope of $(\partial K / \partial T)=1.1 \pm 0.45 \mathrm{ppm} / 100 \mathrm{~K}$ and a jump of $(12 \pm 8) \%$ when crossing the melting point $[9,10]$. In contrast the ${ }^{+}{ }^{+} \mathrm{KS}$ in Cd shows a very pronounced temperature dependence up to the malting point, but practically no change when passing into the liquid state (see Fig. B). At $110 \mathrm{~K}$ we find a singularity 
in the temperature dependence which has been recently identified as a band structure effect related to critical points in the vicinity of the $K$ symmetry point of the hexagonal Brillouin zone[9,11,16].The overall temperature dependence is interpreted in analogy to the analysis of the nuclear Cd KS data $[17]$ as a consequence of phonon-electron interactions which weaken the lattice potential and lead to an increasingly free electron character of the conduction electrons. It would be very interesting if some theorist could be motivated to calculate the concomitant change in the heat of solution for hydrogen. Remarkable is also the absence of a change of $K_{\mu}$ upon melting, quite in contrast to nuclear Cd Knight shift data which show a $+33 \%$ jump upon melting.

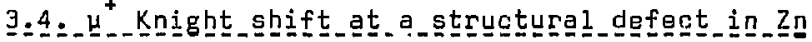

In contrast to the data obtained in polycrystalline $\mathrm{Zn}$, which only showed a very woak temperature depandence, quite a different temperature rependence was found in a single crystal sample (see Fig. 9). At low and at high temoeratures the data are in agrement with the polycrystalline sample results but at intermediate temperatures the KS shows a dramatic drop to large and negative values reaching its extremum at about room temperature. This behavior could not be influenced 
by annealing the sample but it could be induced also in a polycrystalline sample by quenching it rapidly from a temperature close to the melting point down to $77^{\circ} \mathrm{K}[18]$.

The data can be understood in terms of trapping of the $\mu$ * at a second site involving a Knight shift of $-520 \mathrm{ppm} \pm 10 \%$ which sharply contrasts with the value of $68 \pm 5 \mathrm{ppm}$ associated with the regular site. The escape activation energy out of this second site was determined to be $0.5 \mathrm{eV} \pm 5 \%$, i.e. it presents a rather deep trap to the $\mu^{*}$. It was also possible to obtain a rough estimate of the formation energy of this trap from the quenched polycrystalline samples: $0.5 \pm 0.2 \mathrm{eV}$. The escape energy as well as the formation energy suggest that the trap site is a vacancy $[11,18]$. But since it was not possible to anneal this defect by heating, such an identification seems to be ruled out. Another possibility is the formation of a multivacancy cluster or void which is thermally much more stable. Such defects will be formed from single "sancies during the temperature quench, if the quench rates are not to high. Indeed our quench rates were oniy of the order of 80 - $120^{\circ} \mathrm{K} / \mathrm{sec}$. Taking everything together it seems therefore quite likely that the trap site is a multivacancy cluster or small void. Manninen [14] pradicts on the basis of the spherical solid model a KS of $K_{\mu}=+280 \mathrm{ppm}$ for a $\mu^{+}$at a vacancy in $Z n$. It was also found that the KS incr zases with 
the vacancy or microvoid size if the $\mu^{+}$is at the center of this spherical defect [19].

The large negative KS, found experimentally, contradicts these predictions severely, indicating that the change in local electronic structure arcund the $\mu^{+}$by going from the interstitial site to a vacancy cluster is not really understood. Generally,information on the local electronic structure of iydrogen at a defect site appears very important, e.g. in relation to the mechanisms of hydrogen induced corrosion (weakening of metallic binding).

IV. Knight shift in transition metals

So far $K_{\mu}$ was determined in the group $V B$ metals: V.Nb and Ta [4] and in the group VIII metals: Ni, Pd and Pt [20]. We will only discuss here results obtained in the latter three metals as a function of temperature. As an example Fig. 10 shows a plat of $K_{\mu}$ in Pt as a function of the magnetic bulk susceptibility with temperature as an implicit parameter. From such a plot one can determine the temperature independent s-electron induced KS: $K_{s}$ and the d-electron 
induced hyperfine field per unpaired d-electron per atom: $\theta_{h f, d}^{\Omega a}$ which is just the slope of the straight line in Fig. 10.

Tab. II compiles the results obtained in $\mathrm{Ni}, \mathrm{Pd}$ and $\mathrm{Pt}$. We notice that $B_{h f, d}^{R a}$ increases by roughly a factor of 2 in going from $\mathrm{Ni}$ to $\mathrm{Pd}$ and likewise in going from $\mathrm{Pd}$ to Pt. For comparison Tab. II list also d-glectron induced hyperfine fields at the host nuclei. Katayama et al. [21] have shown on the basis of KKR-CPA band structure calculations that the negative hyperfine field at the $\mu^{+}$in $\mathrm{Ni}$ originates from hydrogen-metal bonding states (involving hybridized hydrogen

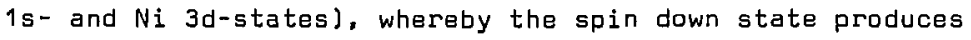
a larger charge density at the $\mu^{+}$than the spin up state.

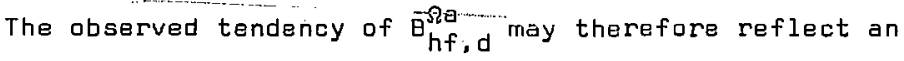
increasing spectral weight of such bonding states. Possible consequences of such a behavior for ather properties of hydrogen in these metals, like local lattice relaxation, heat of solution, etc. must be investigated in the future. It may be recalled in this respect that hydrogen can be dissolved easily in Pd but not in Pt [22]. 


\section{Table II}

Compilation of $\mu^{+}$and nuclear Knight shfit data in Ni, Pd and Pt.

\begin{tabular}{|c|c|c|c|c|c|c|}
\hline & $a\left(A^{\circ}\right)$ & $\begin{array}{l}\text { s-electron } \\
\text { per atom }\end{array}$ & $\begin{array}{c}x_{s} \\
{\left[\mathrm{emu} / \mathrm{cm}^{3}\right]}\end{array}$ & $\begin{array}{c}K_{\mathrm{s}}^{\mu} \\
{[p \mathrm{pm}]}\end{array}$ & $\begin{array}{c}B_{h f, d}^{\Omega_{a}, \mu^{+}} \\
{\left[k G / \mu_{B} / a t\right]}\end{array}$ & $\begin{array}{c}\Omega_{a}, \text { Nucl } \\
h f, d . \\
{\left[k G / \mu_{B} / a t\right]}\end{array}$ \\
\hline $\begin{array}{l}\mathrm{Cu} \\
\mathrm{Ni}\end{array}$ & $\begin{array}{l}3.61 \\
3.52\end{array}$ & $\begin{array}{c}1 \\
0.6\end{array}$ & $\begin{array}{l}1.36 \cdot 10^{-6} \\
0.62 \cdot 10^{-6}\end{array}$ & $\begin{array}{l}80(3) \\
31(27)^{+}\end{array}$ & $\begin{array}{c}-- \\
-1.224(23)^{+}\end{array}$ & $\begin{array}{c}-- \\
-137\end{array}$ \\
\hline $\begin{array}{l}\mathrm{Ag} \\
\mathrm{Pd}\end{array}$ & $\begin{array}{l}4.09 \\
3.89\end{array}$ & $\begin{array}{c}1 \\
0.36\end{array}$ & $\begin{array}{l}0.85 \cdot 10^{-6} \\
0.64 \cdot 10^{-6}\end{array}$ & $\begin{array}{l}114(4) \\
62(13)\end{array}$ & $\begin{array}{c}-- \\
-2.39(11)\end{array}$ & $\begin{array}{l}-- \\
-345\end{array}$ \\
\hline $\mathrm{Au}$ & $\begin{array}{l}4.08 \\
3.92\end{array}$ & $\begin{array}{c}1 \\
0.2\end{array}$ & $\begin{array}{l}0.99 \cdot 10^{-6} \\
0.53 \cdot 10^{-6}\end{array}$ & $\begin{array}{l}64(10) \\
73(15)\end{array}$ & $\begin{array}{c}-- \\
-5.03(43)\end{array}$ & $\begin{array}{c}-- \\
-1180\end{array}$ \\
\hline
\end{tabular}

+ revised value 
V. Knight shift in $\beta-P d H x$

So far all reported investigations concerned a single $\mu^{+}$(or protonl in the pure metal. This corresponds to an infinite dilute concentration of hydrogen in a metal, that is, we are within the $\alpha$-phase of the metal hydragen system. The bulk electronic structure of the host metal is only locally disturbed and effects of hydrogen-hydrogen interactions are altogether absent. The situation is quite different in the other ragime of high hydrogen concentrations, i.e. in a metal hydride.

The only previous $\mu S R$ KS study in a metal hydride was performed in the system $\operatorname{LaNi}_{5} \mathrm{H}_{x}$ [23]. Here we review some more recent investigations in $B-\mathrm{PdH}_{x}$ for concentrations of $x=$ $0.59,4.70,0.75,0.81$ and 0.86 . Except for $x=0.59$ the KS is temperature independent [20] reflecting the fact that also the bulk magnetic susceptibility becomes practically temperature independent above $x=0.60$ [24]. Fig. 11 displays the temperature ranges investigated for the various $\mathrm{H} / \mathrm{Pd}$ ratios and the average KS found for each ratio. For $x=0.59$ one finds a significunt drop in $K_{\mu}$ when going from room temperature and above to $4.2 \mathrm{~K}$. This is probably related to a transition from the $\beta$-phase to the mixed $\alpha+\beta$ phase just slightly below room temperature. This has to be investigated in more detall in the future, but if the change in $K$ is really asso- 
ciated with a phase transition an interesting field of further studies is opened up.

Fig. 12 displays the observed shifts corrected for a diamagnetic contribution of $-20 \mathrm{ppm}$, as a function of hydrogen concentration. Indicated are also two points stemming from proton KS measurements by NMR [25]. Excellent agreement between the NMR and $\mu S R$ data can be stated, indicating that isotope effects are only of minor importance. It appears that the KS in $\beta-P d H_{x}$ rises linearly with the H-concentration. Also indicated in Fig. 12 is the temperature independent s-electron induced $K_{s}$ obtained in pure Pd (see Tab. II). Since the d-band is completely filled in $\mathrm{PdH}_{x}$ for $x \geq 0.6$ and falls below the fermi energy it is tempting to trace the observed temperature independent $\mu^{+} \mathrm{KS}$ in $\beta-\mathrm{PdH}_{x}$ back to s-glectrons too. Obviously then $K_{s}$ is quite different in pure $P d$ and in its hydride. The Pauli spin susceptibility $x_{s}$ af the hydride is estimated to be $1.8 \times 10^{-6} \mathrm{emu} / \mathrm{cm}^{3}$ [26] which is much larger than the value estinated for pure Pd (see Tab Tab. II). Hence, one would have expected a much larger $\mathrm{K}_{\mathrm{s}}$ in the $B$-hydride phase. Dn the other hand there is no indication that $x_{5}$ is dependent on $x$ for $x \geq 0.70$ [26]. The $x-d e-$ pendence of $K_{s}$ must therefore be of a different origin [25]. 
The situation becomes even more puzzling if one compares the Knight shift data with predicitions obtained from proton Korringa relaxation rate $\left(T_{1 e} \times T^{-1}\right.$ measurements $[27,28,29]$ by applying the Korringa relation. The predicted values are also displayed in Fig.12. There segms to be no $x$-dependence between $x=0.70$ and 0.80 , essentially in accordance with a constant $X_{s}$. A remarkable discrepancy between the derived numbers and the measured shifts in $\mathrm{PdH}_{x}$ is apparent. These conflicting results can be reconciled if one postulates two $x$-dependent contributions $K_{1}, K_{2}$ cancelling partially in $K_{\mu}$ (and $K_{p}$ ) but adding constructively in the Korringa relaxation rate. However, no obvious interpretation of two contributions is at hand. Another possibility is to assume that an abnormally large and $x$-dependent diamagnetic shielding reduces the total knight shift without affecting the Korringa relaxation rate. A required diamagnetic screening of the order of -65 ppm would point to a very peculiar elactron distribution around the $\mu^{+}$or proton, which is not indicated so far by other measurements. 


\section{Summary}

What has been learned from the present $\mu^{*}$ Knight shift studies about the local electronic structure of this hydrogenlike impurity? The conclusions may be summarized as follows:

a ) hydrogen_in_simple__nontransition_metals

A screened proton picture, underlying the spherical solid model calculations, provides an adequate model to explain the gross features of the observed KS systematics. In addition to scattering states bound states seem to be very important in producing a negative contribution to the KS which is essential in reproducing the data. The influence of the host neighbor potentials resulted in a further enhancement of the spectral density of the bound states on the expens. of continuum scattering states. Implications on other properties like the heat of solution of hydrogen in these metal. have still to be investigated theoretically. Lacking agr: ... ment in detail indicates that further refinemerts are necessary, perhapg by applying more realistic cluster or band structure calculations, including also the effect of lattice relaxation around the impurity. The observation of anisotropies in the $\mu^{+} K S$, notably in Be, suggests that also non s-electron like states can contribute 
to the local electronic structure in a non negligible way. This needs to be explored further in future experimental investigations as well as in theorotical studies. Unexplained is the drastic change of the $\mu^{+} \mathrm{KS}$ in $\mathrm{Zn}$ when trapped in a structural defect (probably a multivacancy clusterl.

\section{ㄴ) Lydrogen in d-electron transition metals}

The $\mu^{+}$KS studies in $\mathrm{Ni}, \mathrm{Pd}$ and Pt show that d-electrons play an important role in the formation of the total spin density at the $\mu^{+}$. This may be interpreted in various ways. The KKRband structure calculations of Katayama et al. [21] suggest that this is the result of the formation of bonding states involving hybridized hydrogen is and host metal d-wave functions. This would support the covalent hydrogen model suggested for transition mecal hydrides [30]. What is needed now are more calculations extended to Pd and Pt in order to understand also the observed trends in $\theta_{h f, d^{\Omega}}$.

\section{c) metalal hydrides}

The as yet insomplete measurements in $\beta-\mathrm{PdH}_{x}$ promise to elucidate various properties: (i) change of the local charge density at the $\mu^{+}$with the hydrogen concentration, (ii) possible influence of electrons from the fully occupied 
d-band taking both the $K S$ and $T_{1 e^{-d a t a}}$ into acrount, (iii) behavior of the local electronic structure at the boundary between different phases. We hope to have more on this by the time of the next conference on hydrogen in metals.

\section{Acknowledgements}

The experiments on $\mathrm{PdH}_{x}$ have been performed in collaboration with L. Schlapbach and his group. This work has been supported in part by the Schweizerisches Institut für Nuklearforschung, Villigen. 


\section{References}

[1] see e.g. R.M. Cotts in Hydrogen in Metals, Vol. 1, Eds. G. Alefeld, J. Välkl (Springer Verlag, 1978).

[2] P. Jena, in Treatise on Materials Science and Technology, Vol. 21, p. 351 (Academic Press, 1981).

[3] E. Zaremba and D. Zobin, Phys. Rev. B22 (1980) 5490.

[4] A. Schenck, Helv. Phys. Acta 59 (1981) 471.

[5] A. Schenck in Electronic Structure and Properties of Hydrogen in Metals. Eds. P. Jena and C.B. Satterthwaite (Plenum Press, 1983).

[6] M. Camani et al., Phys. Rev. Lett. 42 (1979) 679.

[7] F.N. Gygax et al.. Proceed. Yamada Conf. HSR (1983), Eds. T. Yamazaki, K. Nagamine (to be published in Hyperfine Interactions).

[8] W. Studer et al., loc. cit, ref. 7.

[9] W. Studer, thesis (ETH Zürich, 1983) unpublished.

[10] F.N. Gygax et al., Phys. Rev. Lett. 51 (1983) 505.

[11] W. Studer et al., loc.cit. ref. 7.

[12] 0. Hartmann et al., Hyperfine Interactions 6 (1979) 47. 
[13] J.H. Brewer et al., Solid State Communic. 46 (1983) 863.

[14] M. Manninen, Phys. Rev. B27 (1983) 53.

[15] G.G. Carter, L.H. Bennett, D.J. Kahan, Metallic Shifts in NMR, Part 1, Progr. Mat. Sci. Vol. 20 lPergamon Press, 1977).

[16] W. Studer et al., to be published.

[17] R.V. Kasovski, Phys. Rev. 187 (1969) 891.

[18] F.N. Gygax et al., loc, cit. ref. 5, P. $\underline{407}$.

[19] R.M. Nieminen et al., loc.cit. ref. 7 .

[20] F.N. Gygax et al., Proceed. Int. Conf. Hyperf. Interactions (Groningen, 1983), to be published jin Hyperf. Interactions.

[21] H. Katayama et al., Solid State Communic. 29 (1979) 431.

[22] J.K. Norskov, Phys. Rev. 旦6 (1982) 2875.

[23] F.N. Gygax et al.. in Recent Developments in Condensed Matter Physics, Vol. 2, Eds. J.T. Devreese et al.. (Plenum Press, 1981).

[2A] H. Frieske and E. Wicke, Ber. Bunsenges. Phys. Chem. 77 (1973) 48 . 
[25] P. Brill and J. Voitländer, Ber. Bunsenges. Phys. Chem. I7 (1973) 1097 .

[26] H.C. Jamiason et al., ]. Phys. F2 (1972) 323.

[27] G.K. Schoep et al., Physica 75 (1979) 297.

[28] D.A. Cornell et al., J. Less-Common Metals 39 (1)75) 43.

[29] C.L. Wiley et al., Phys. Rev. 昂7 (1978) 3462.

[30] C.D. Gelatt st al., Phys. Rev. B17 (1978) 1940. 


\section{Figure captions}

\section{Figure.1}

Semilogarithmic plot of the induced hyperfine field $B_{h f}^{\Omega a}=\mu_{B} \cdot K_{s} / x_{a t}$ versus the molar electronic specific heat.

Figure_e 2

Partial wave decomposition of the electron density at the $\mu^{+}$ at a tetrahedral site in Cs from spherical solid model calculations. The datted dashed line is the electron density (spin up or down) from a nonpolarized jeliium model [14].

Eigure_3

Spherical solid model predictions for $K_{\mu}$ in monovaler. metals versus the density parameter $r_{s}[14]$.

\section{Figure 4}

Spherical solid model predictions for $K_{\mu}$ in divalent metals. The upper values are for $\mu^{+}$at the octahedral interstitial site, tha lower ones at the tetrahedral site [14]. 
Figure. 5

Comparison of spin density enhancement factors $\rho_{S}(0)$ from the spherical solid model calculation [14] and from the experimental KS values using experimental spin susceptibility data [4].

Figure $-\underline{6}$

Anguiar dependence of $K_{\mu}$ in $B e$ at $293 \mathrm{~K} . \theta$ is the angle between the crystalline c-axis and the applied field [9].

Eigure ?

Angular dependence of $K_{\mu}$ in $C d$ at $60 \mathrm{~K}$ and at $293 \mathrm{~K}$. $\theta$ is the angle between the crystaline c-axis and the applied field [1 18$]$.

Figure - 은

Temperature dependence of $k_{\mu}$ in polycrystalline Cd [11]. 


\section{Eigure음}

Temperature dependence of the apparent $k_{\mu}$ in a single crystal sample of $\operatorname{Ln}[10]$.

Figure $\underline{1} \underline{0}$

Plot of $K_{\mu}$ versus magnetic susceptibility in Pt. Temperature is an implicit parameter [20].

Tigure 11

Temperature range of $K_{\mu}$ measurements in $\mathrm{PdH}_{x} \cdot K_{\mu}$ is temperature independent except for $x=0.59$.

\section{Eigure 12}

Plot of $K_{s}$ versus hydrogen concentration $x$ in ${ }^{\circ} \mathrm{dH}_{x}$. Indicated are also results from proton KS measurements [25] and calculated values (via the korringa relation) from T pel $^{\text {-relaxation }}$ data $[27-29]$. 


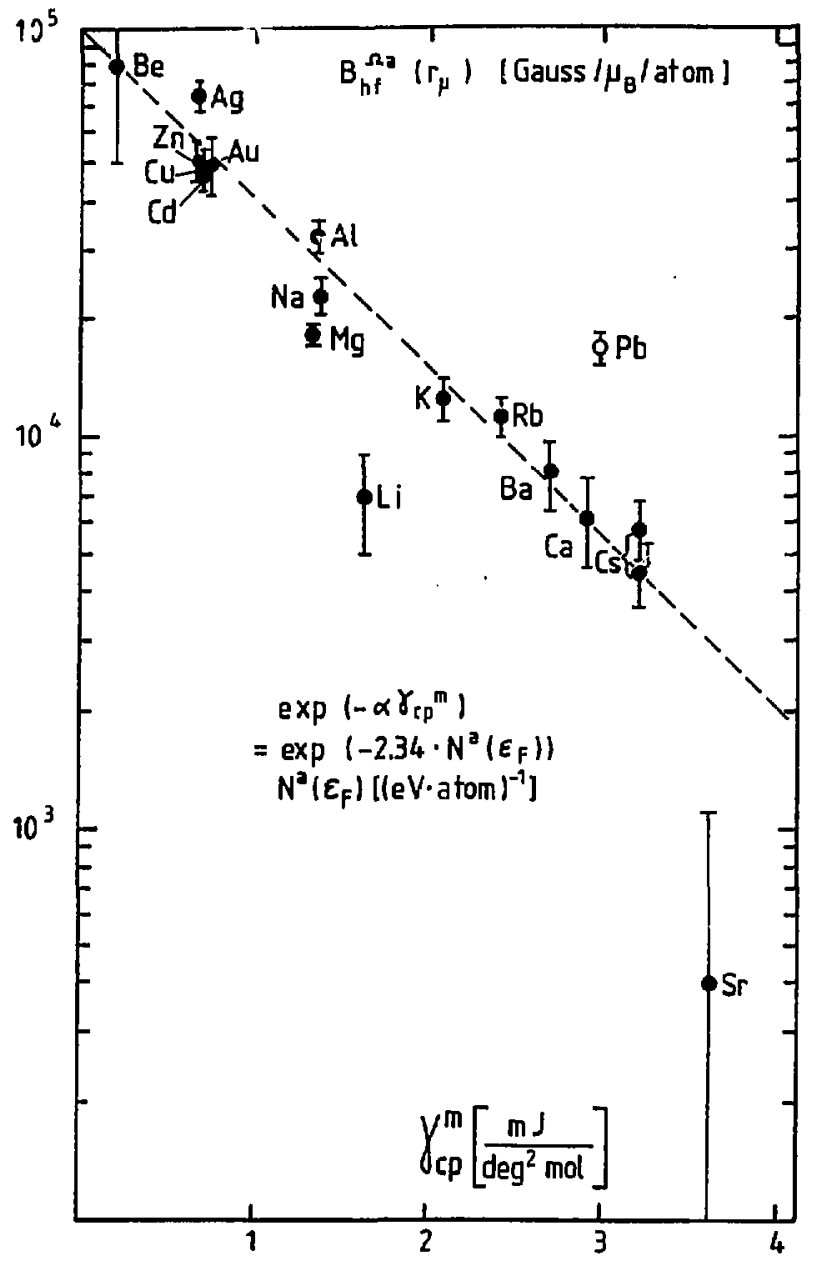

Fig, 1 


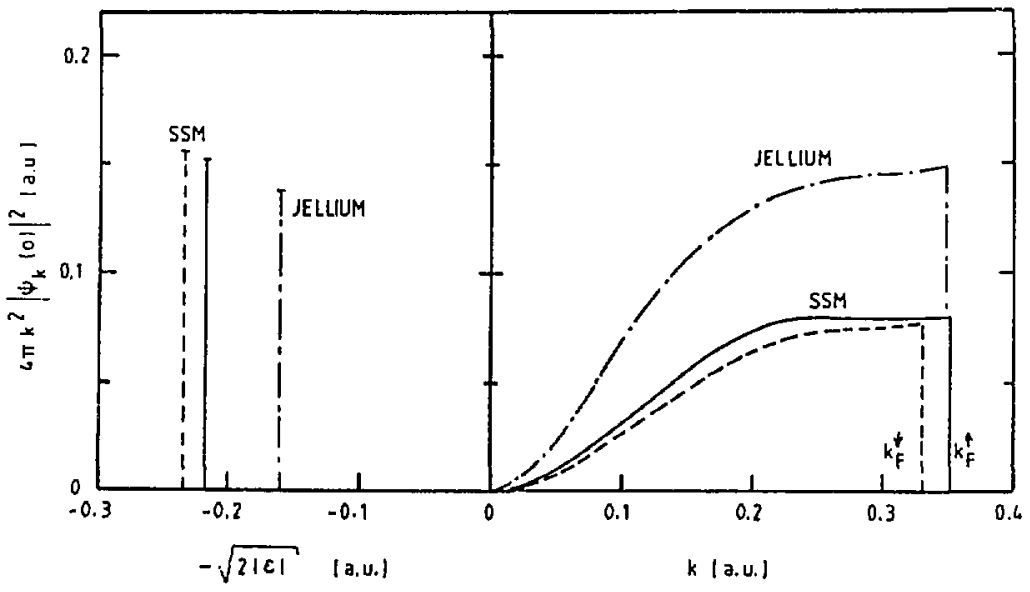

Fig. 2 . 


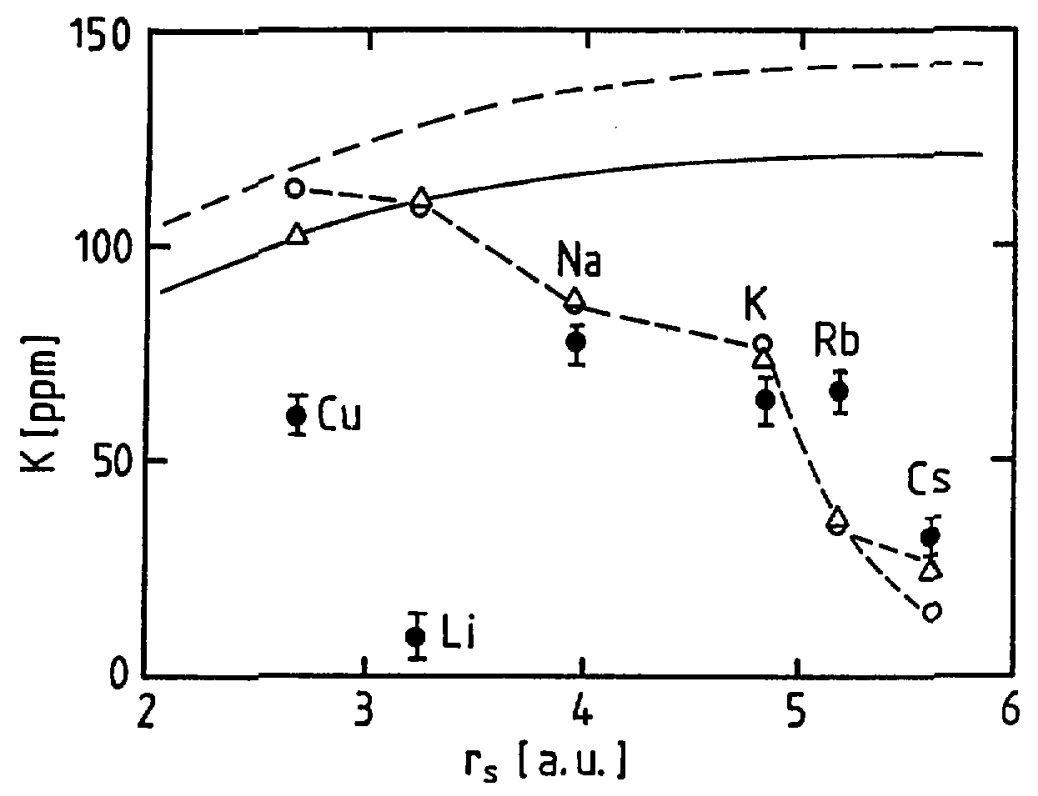

Fig. 3 


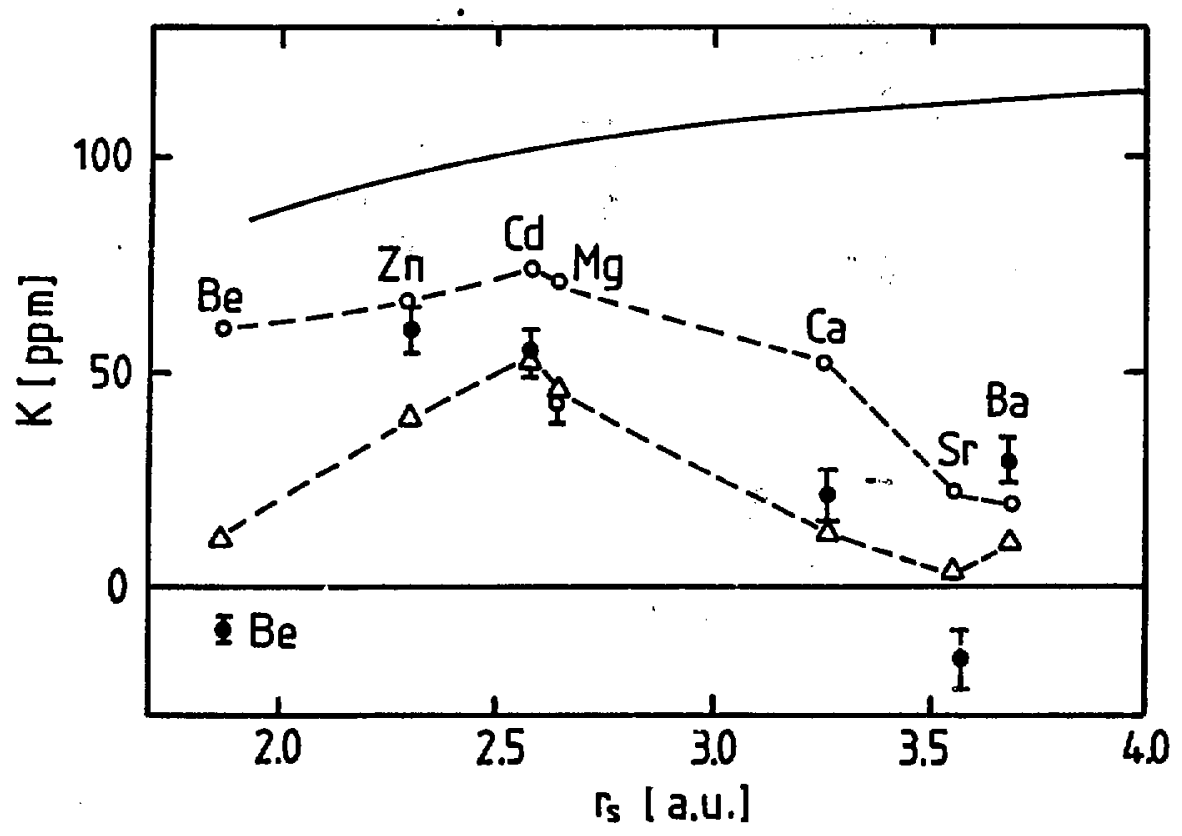

Fig. 4 


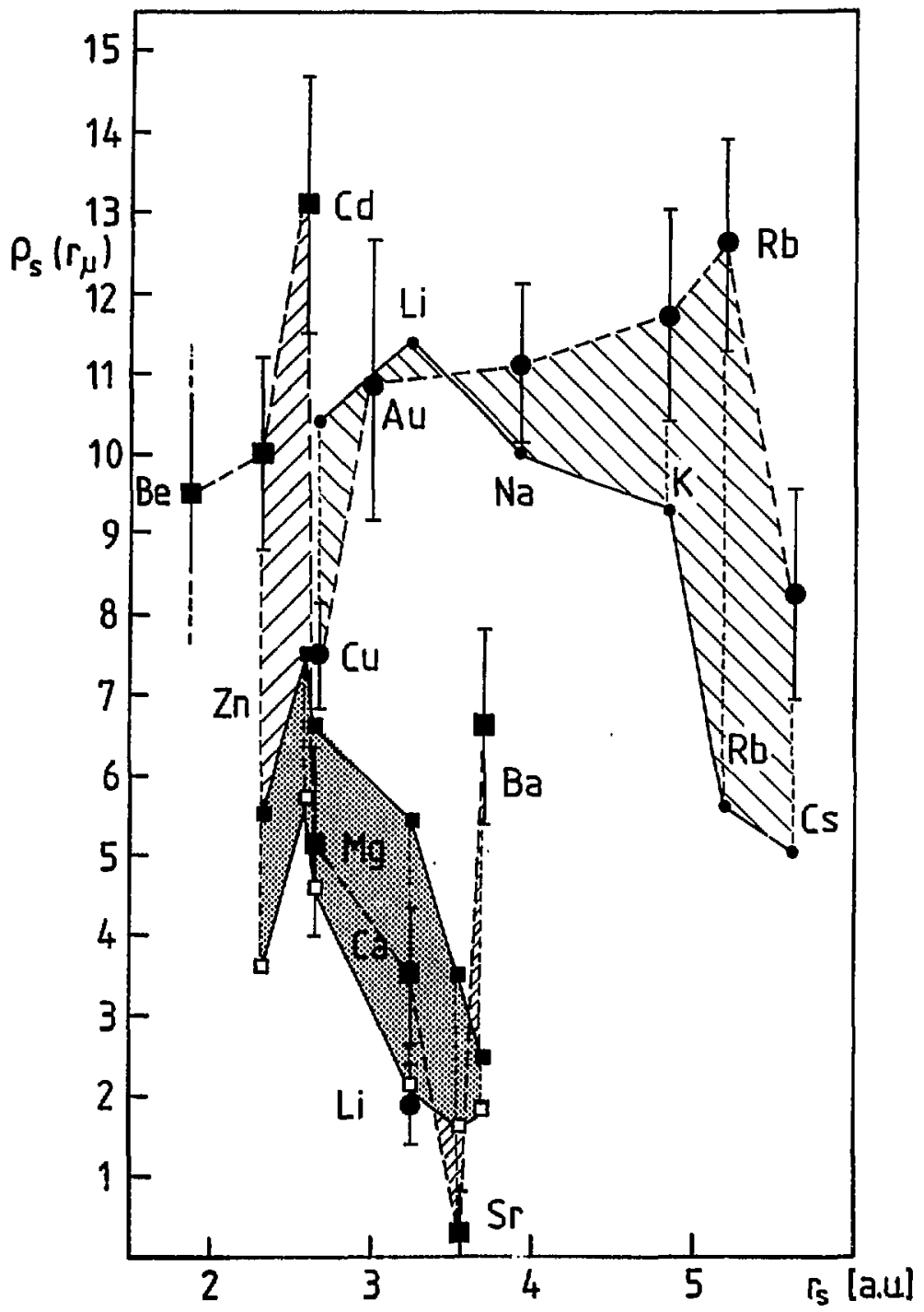

Fig. 5 


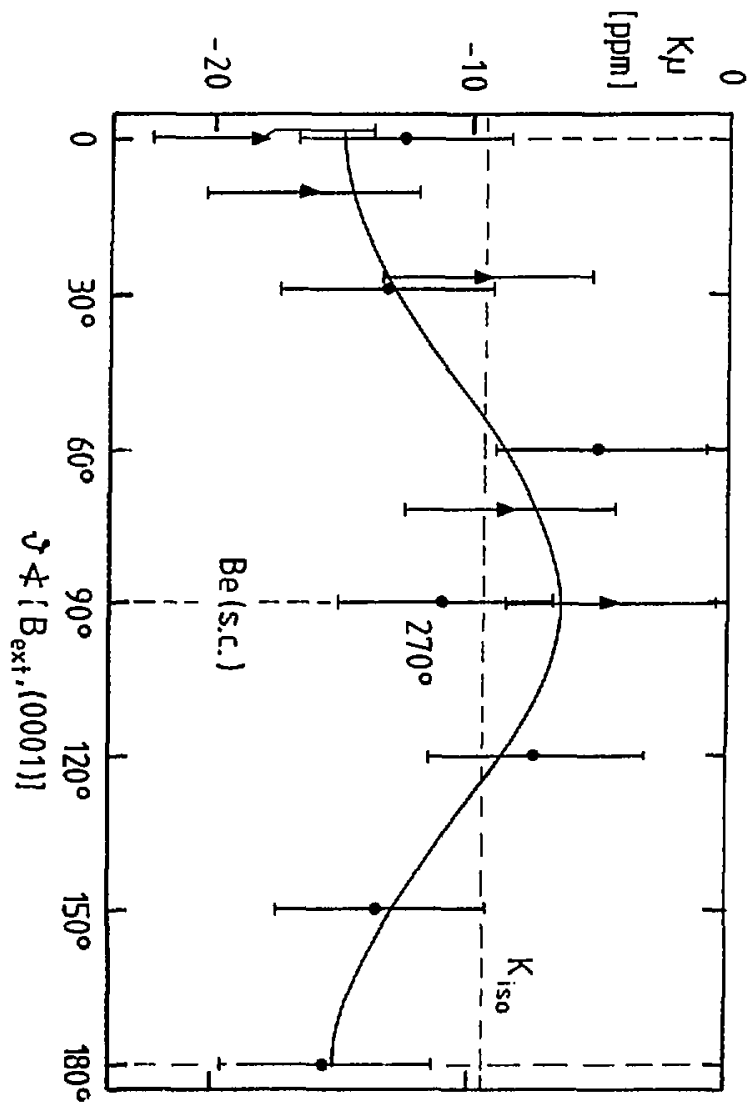

Fig. 6 

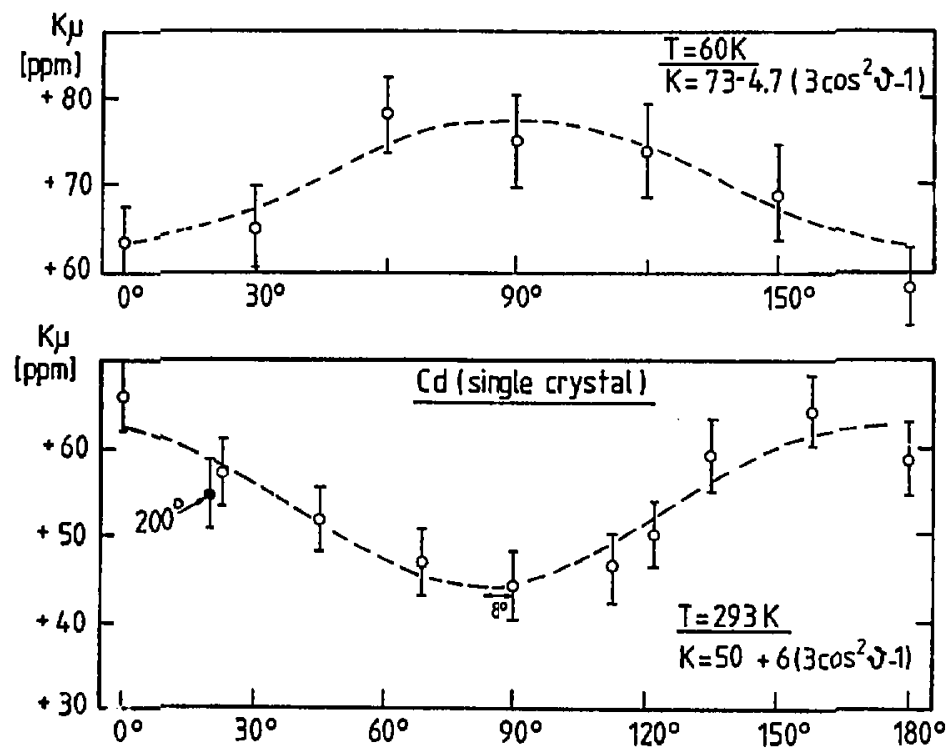

Fig. 7 


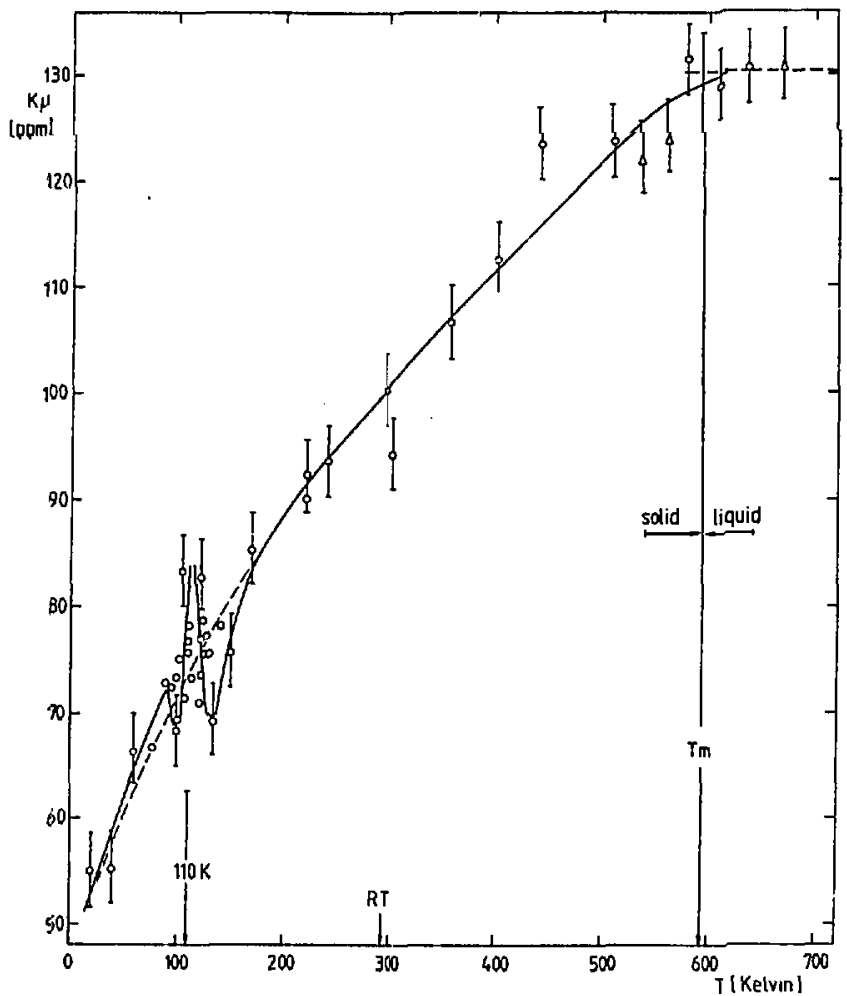

Fig. 8 


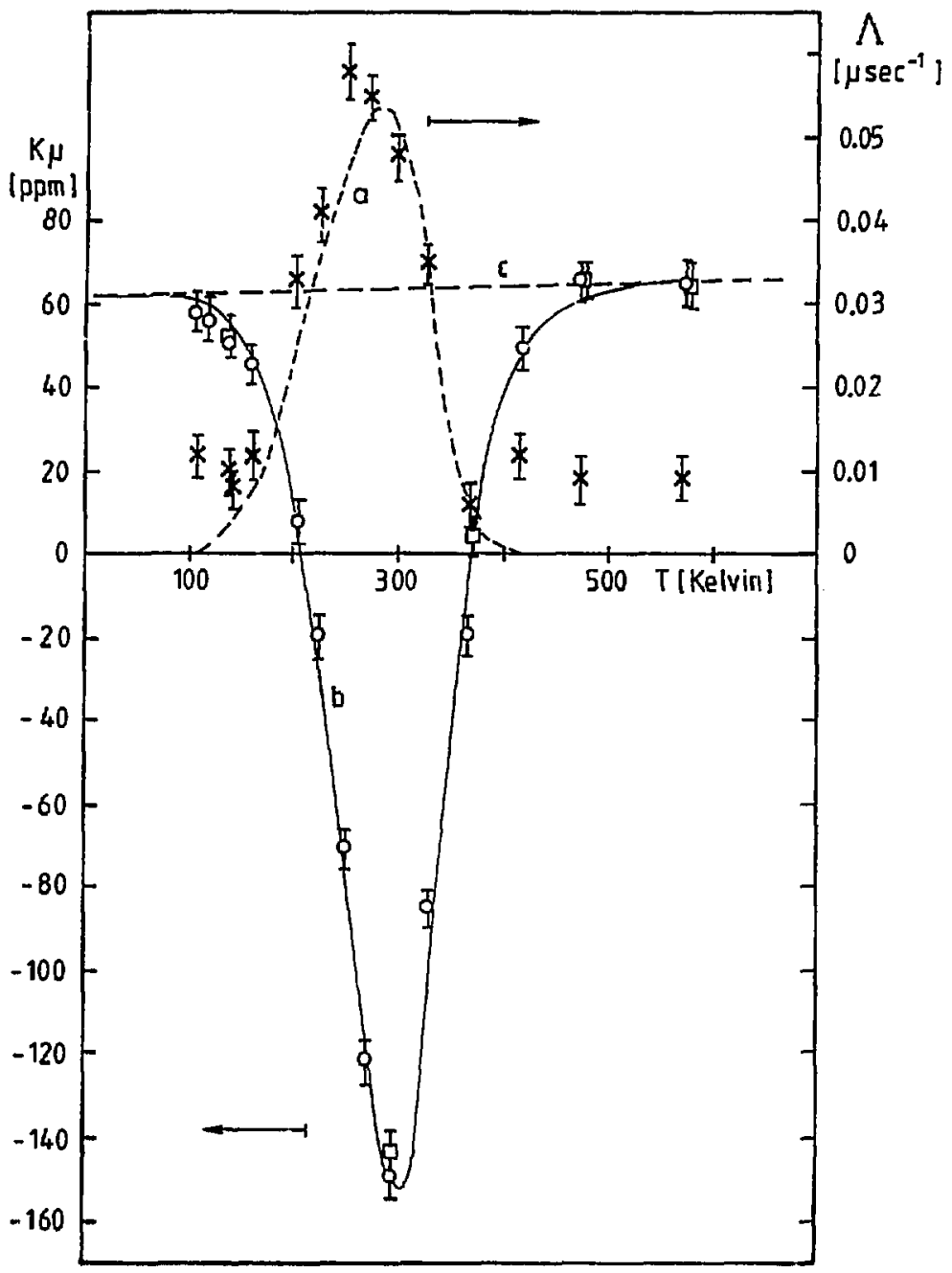

Fig. 9 


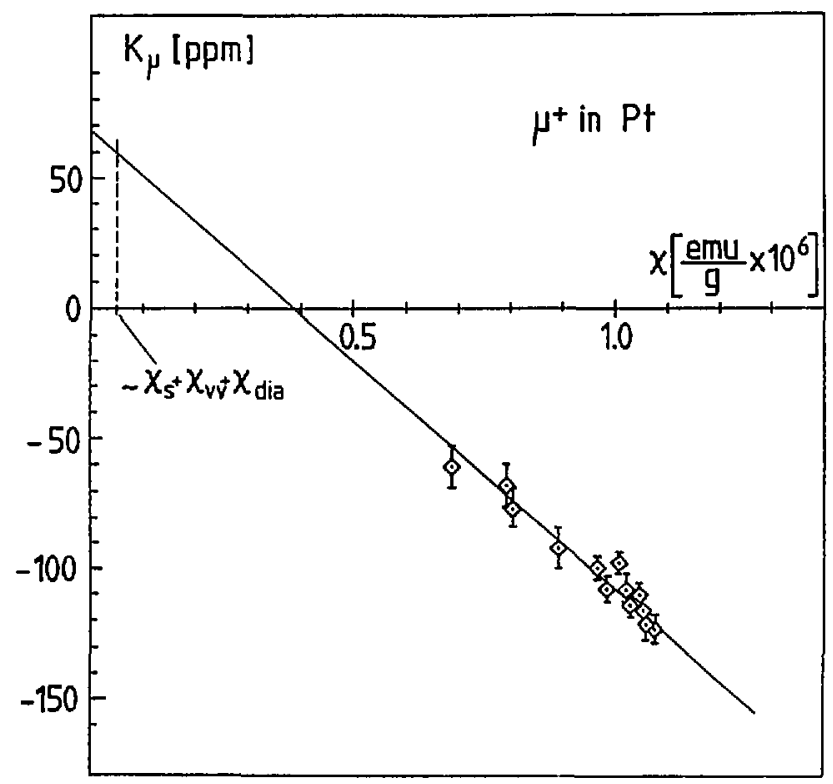

Fig. 10 


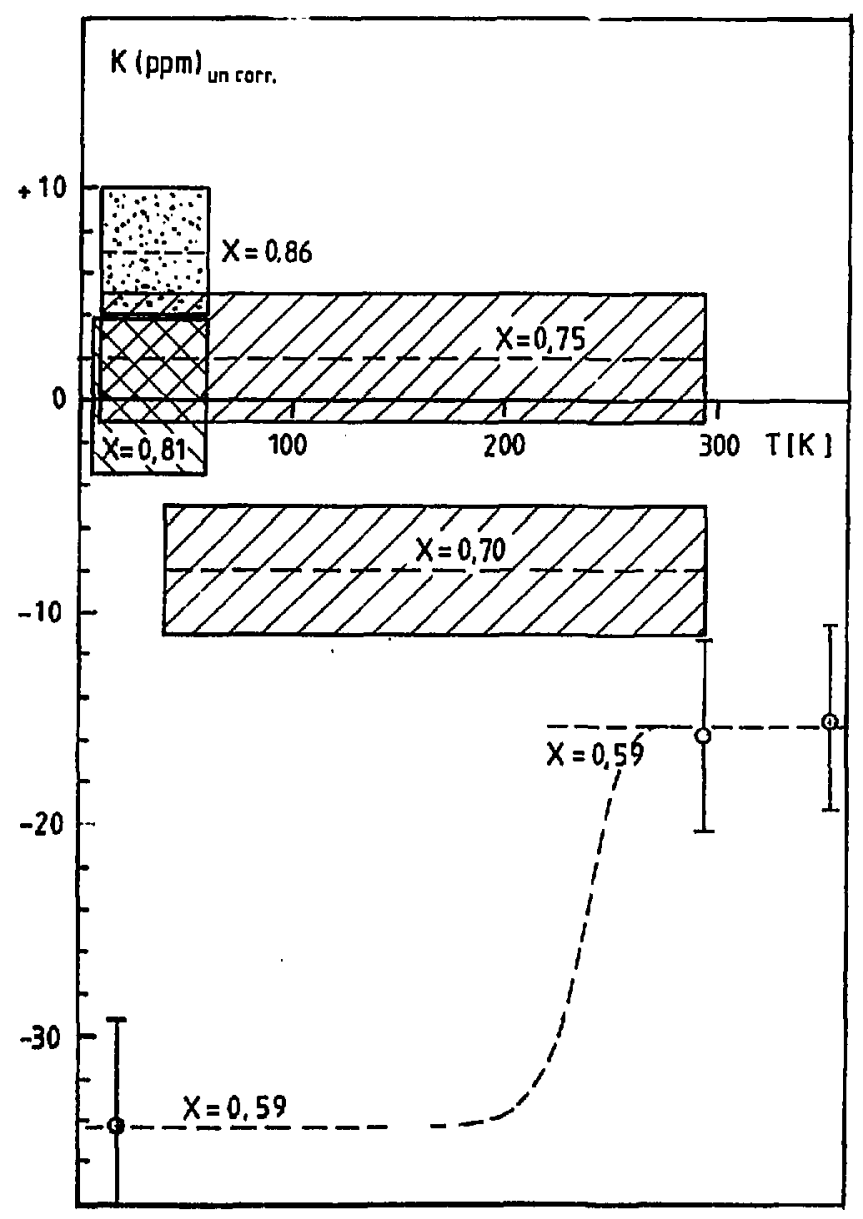

Fig. 11 


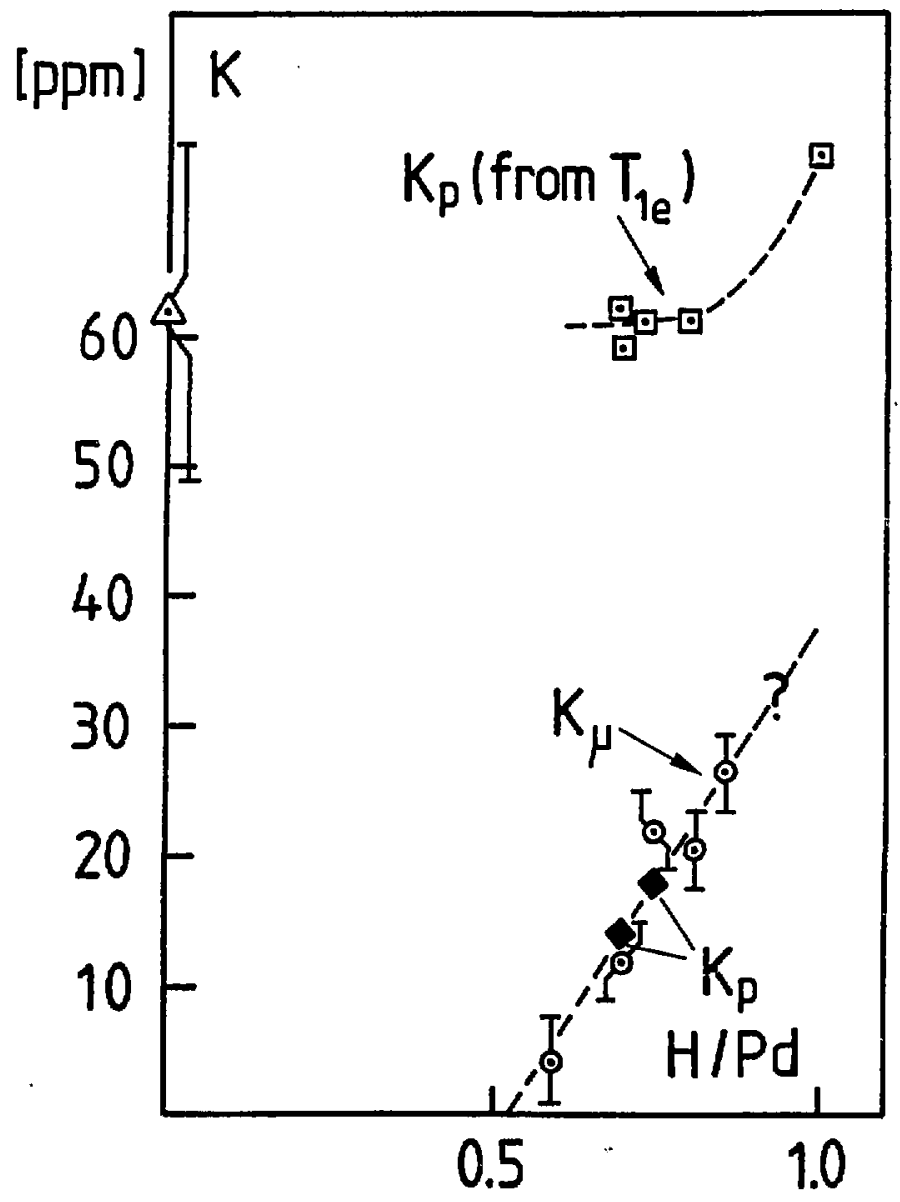

Fig. $\quad 12$ 
Quantum Diffusion of Positive Mons in Iron

E. Yagi (a) G. Fli\}, K. Furderer, N. Haas, D. Herlach,

$$
\text { J. Major }{ }^{(b)} \text {, and A. Seeger }
$$

Universität Stuttgart, Institut für Theoretische und Angewandte Physik, and Max-Planck-Institut für Metallforschung, Institut für Physik,

Postfach 800665, D-7000 Stuttgart, Germany

and

W. Jacobs, M. Krause, M. Krauth, H.-J. Mundinger, and H. Orth Physikalisches Institut der Universität Heidelberg, D-6900 Heidelberg 1 , Germany

(Received

The longitudinal mon-spin relaxation rate $\underline{\Gamma}_{1}$ of a spherical $\alpha$-iron single crystal in an applied magnetic field of $4.5 \mathrm{~T}$ parallel to $\langle 111\rangle$ shows a maxinum at $\underline{T}=3.7 \mathrm{~K}$. Near the maximum $\Gamma_{1}(T)$ is in agreement with a mon diffusivity $\underline{D}^{\mu^{+}}$proportional to the temperature $\underline{T}$ and hence with one-jinonon-assisted tunnelling. The results strongly support the view that the stable muon site is the tetrahedral interstice. Below about $3 \mathrm{k}$ evidence for metastable occupancy of a second type of site has been found.

PACS numbers: $66.30 . \mathrm{Jt}, 76.60 . \mathrm{Jx}, 76.90 .+\mathrm{d}$

The study of the diffusivity $\underline{D}^{\mu+}$ of positive muons $\left(\mu^{+}\right)$in metals has been one of the main incentives for the development of the theory of quantum diffusion in crystals and, at the same time, its main testing ground. ${ }^{1}$ The longitudinal spin relaxation of $\mu^{+}$in $\alpha-F e$ is particularly weli suited for quantitative studies of $\underline{D}^{\mu \pm}(T) \cdot{ }^{3-4}$ Measurements of the longitudinal relaxation rate Ifare insensitive against trapping effects due to impurities or other imperfections ${ }^{5}$ and hence superior to transverse relaxation 
measurements. In studying $\alpha-F e$ we take advantage of 1 ts ferromagnetism and the lower-than-cubic point symmetry of 1ts interstices. This leads to strong dipolar magnetic fields $\vec{B}_{\text {dip }}$ acting on the magnetic moments of the $\mu^{+}$located on interstitial sites. When the $\mu^{+}$diffuse by jumping between neighbouring interstices, the aipolar fields felt by the $\mu^{+}$magnetic moments change. Provided the anplied magnetic field $\vec{B}_{a p p l}$ is chosen appropriately, this affects the muon polarization so strongly that $\Gamma_{1}$ can be determined over a very wide temperature range.

In the body-centred cubic (bcc) structure of a-Fe the interstitial sites most likely to be occupied by $\mu^{+}$, the tetrahedral (T) and octahedral $(0)$ interstices, possess tetragonal point symmetry. In the demagnetized state the local magnetization $\vec{M}_{\text {domain }}$ is parallel to $\langle 100\rangle$. The dipolar fieldsat 0 or $T$ sites $^{6}$ are then parallel to $\overrightarrow{\underline{M}}_{\text {domain' }}$ so that $\underline{I}_{1} \equiv 0$. Consequently, the longitudinal relaxation measurements must be carried out on single crystals with $\vec{M}_{\text {domain }}$ rotated away from <100> by a suitably oriented and sufficiently strong external field $\vec{B}_{a p p l}$.

A complete theory of spin relaxation due to the topping motion of $\mathrm{H}^{+}$between the above-mentioned sites in ferromagnets has been worked out by seeger and Monachesi ${ }^{7}$. If a single correlation time $\tau_{c}=I_{c}(\underline{I})$ suffices to describe the $\mu^{+}$diffusion, $I_{1}$ is given by

$$
\underline{\Gamma}_{1}=2 \hat{\Gamma}_{1} \underline{I}_{c} / \underline{I}_{c}\left(1+\underline{w}_{\mu}^{2} \underline{I}_{c}^{2}\right)
$$

where $\underline{t}_{\underline{\omega}} \underline{\mu}_{\mu}$ denotes the Zeeman splitting of the $\mu^{+}$spin levels. Due to the compensation of the Lorentz field $\underline{\mathrm{B}}_{\text {LORENTz }}=\underline{\mathrm{O}}_{\mathrm{O}} \mathrm{M}_{\mathrm{sp}} / 3$ $\underline{M}_{\mathrm{sp}}=$ spontaneous magnetization and the demagnetizing field, for magnetically saturated spherical samples 


$$
\underline{\omega}_{\mid \downarrow} \equiv \underline{Y}_{\mu} \underline{B}_{\mu}=\underline{Y}_{\mu}\left(\underline{B}_{\mathrm{app} 1}+\underline{B}_{\mathrm{FERMI}}\right)
$$

holds $\underline{Y}_{\mu}=8.561 \cdot 10^{8} \mathrm{rad} \mathrm{s}^{-1} \mathrm{~T}^{-1}=$ gyromagnetic ratio of the muon, $\vec{B}_{\text {FERMI }}=$ contact field due to the spin polarization of the conduction electrons). According to Eq. (1), $\underline{\Gamma}_{1}$ shows a maximum $\hat{\underline{\Gamma}}_{1}$ at that temperature $\hat{\underline{T}}$ at which

$$
\underline{\omega}_{\mu} \underline{\tau}_{c}(\underline{\hat{T}}) \equiv \underline{\omega}_{\mu} \hat{\underline{\tau}}_{C}=1
$$

$\hat{\Gamma}_{1}$ depends on the crystallographic orientation of $\underline{\underline{B}}_{a p p l}$ and assumes its maximum value

$$
\underline{\hat{\Gamma}}_{1}^{\langle 111\rangle}=\frac{1}{4}\left(\underline{\underline{Y}}_{\mu} \underline{B}_{\mathrm{dip}}^{\|}\right)^{2} \underline{\hat{\tau}}_{c}
$$

for $\underline{\vec{B}}_{\mathrm{app} 1}$ along $\langle 111\rangle$. If $\underline{\omega}_{\mu}$ is known/ we may use Eqs. (3) and (4) to deduce $\left|{ }_{\text {dip }}\right|$, which is characteristic of the sites occupied by the muons.

According to Teichler, Seeger and Schmidt ${ }^{\theta}, 9$, in bcc metals the low-temperature muon hopping should be governed by one-phonon incoherent tunnelling processes. This mechanism yields $\underline{\underline{\mu}}^{\mu^{+}} \propto \underline{T}$ and hence

$$
\underline{\tau}_{C}=\underline{B}^{-1} \text {, }
$$

where $\underline{B}$ is a constant determined by the muon-phonon interaction. Insertion of Eq. (5) into Eq. (1) gives us, together with Eq. (4),

$$
1 / \underline{T} \Gamma_{1}^{\langle 111\rangle}=\underline{A}+\underline{B} / \underline{T}^{2}, \underline{T} / \underline{\Gamma}_{1}^{\langle 111\rangle}=\underline{B}+\underline{A} \underline{T}^{2}
$$

with $\underline{A}=2 / \underline{B}\left(\underline{Y}_{\mu} \underline{B}_{d i p}^{\|}\right)^{2}$ and $\underline{B}=2 \underline{B}\left(\underline{w}_{\mu} / \underline{Y}_{\mu} \underline{B}_{d i p}\right)^{2}$. 
At SIN, Villigen, Switzerland, we have measured $\Gamma_{1}$ on a spherical $\alpha-F e$ crystal in the ranges $300 \mathrm{~K} \geq \underline{T} \geq 0.85 \mathrm{~K}$ and $4.9 \mathrm{~T} \geq \mathrm{B}_{\mathrm{appl}} \geq 1.2 \mathrm{~T}$. For the lowest temperatures, the apparatus $5 \times 11$ was equipped with a large-impedance ${ }^{3}$ He evaporation cryostat. Operzted with ${ }^{4} \mathrm{He}$ as cryogenic fluid, the base temperature measured with a Ge resistor at $\underline{B}_{\text {appl }}=0$ was $0.85 \mathrm{~K}$. The Ge resistor and a carbon glass sensor were calibrated between $8.3 \mathrm{~K}$ and $0.85 \mathrm{~K}$ in applied fields $2.0 \mathrm{~T} \leq$ Bappl $_{\mathrm{a}} 4.5 \mathrm{~T}$, using $\mathrm{a}$ $\mathrm{SrTiO}_{3}$ capacitor for temperature control.

From measurements between $300 \mathrm{~K}$ and $4 \mathrm{~K}$ the following conclusions relevant for the present work have been drawn $5,12,13$.

(i) The dependence of $\Gamma_{1}$ on the crystallographic direction of $\vec{B}_{\text {appl }}$ is in excellent agreement with exclusive occupancy of sites of tetragonal symmetry down to $6 \mathrm{~K}$, the lowest temperature at which the orientation dependence of $I_{1}$ was investigated.

(ii) Height and position of the maximum of $I_{1}(\underline{T})$ at $32 \mathrm{k}$ (Fig. 1) are field-independent between $1.2 \mathrm{~T} \leq$ Bappl $\leq 4.9 \mathrm{~T}$; sance it cannot be the Inaximum associated with (3). It is attributed to a transition from exclusive occupancy of $\mathrm{T}$ sites below about $15 \mathrm{~K}$ to a thermal-equilibrlum distribution of $\mu^{+}$on $T$ and $O$ sites at high temperatures.

(iii) Below about $20 \mathrm{~K}$ the dependence of $\Gamma_{1}$ on $\underline{B}_{\text {appl }}$ becomes noticeable (Fig.1), Indicating that we might be approaching the maximum assoriated with (3).

FIg. 1 shows longitudinal relaxation rates $\Gamma_{1}^{\langle 111\rangle}$ recently determined between $8.3 \mathrm{~K}$ and $0.85 \mathrm{~K}$ by fitting single exponentials to the measured relaxation functions at times $\geq 60 \mathrm{~ns}$ after $\mu^{+}$ implantation (full symbols) together with the earlier results 5,12 
(open symbols and crosses). The data at $\underline{B}_{\text {appl }}=4.5 \mathrm{~T}$ satisfy (6) quite well (F1g.2), indicating that the maximum $\hat{\Gamma}_{1}^{\langle 111\rangle}=7.5 \cdot 10^{6} \mathrm{~s}^{-1}$ at $\hat{\underline{T}}=3.7 \mathrm{~K}$ is indeed the maximum predicted by $(1)$ at $\underline{\omega}_{\mu} \underline{\tau}_{c}=1$. The dashed lines in Fig.1 represent the fit of Fig.2 for Bapol $_{\text {a }}=$ $4.5 \mathrm{~T}$ and the corresponding curve for $\underline{B}_{\text {appl }}=2.0 \mathrm{~T}$ as calculated from (1) and (2) with the same parameters and $\underline{B}_{\text {FERMI }}=-1.126 \mathrm{~T}$. The deduction of B $_{\text {FERMI }}$ (required in order to obtain $\underline{\omega}_{\mu}$, $\hat{\underline{\tau}}_{c}=\underline{\omega}_{\mu}^{-1}$ and, with Eq. (4), (Bلdip $\left.{ }^{2}\right)$ from the spin precession frequency in zero applied field,

$$
\underline{\omega}_{\mu}^{\circ}=\underline{\gamma}_{\mu}\left(\underline{B}_{\text {LORENTZ }}+\underline{B}_{\text {FERMI }}\right) .
$$

needs some discussion. The tenperature dependence of $\left|\underline{\omega}_{1}^{0}\right|^{14}$ (inset of Fig.1) shows a kink at about $6 \mathrm{k}$, for which a satisfactory interpretation has not been given. For the main conclusions of the present paper it is irrelevant whether the full-line or the dashed-line extrapolation (both Iinear in $\underline{T}$ ) of $\underline{\omega}_{\mu}^{\circ}$ is used since the uncertainty in $\underline{\omega}_{\mu}$ is not more than \pm 0.28 . A convincing explanation for the kink is, nevertheless, desirable. The following proposal ties together several observations in the present experiments.

In accordance with (i) and (ii) we take the full line in the inset of $\mathrm{Fig.1}$ as representing the muon spin precession frequency $\underline{\omega}_{\mu}^{T}$ at $T$ sites. The extrapolation to low temperatures gives us $\left|\underline{\omega}_{\mu}^{T}\right|=3.28 \cdot 10^{8} \mathrm{rad} \mathrm{s} \mathrm{s}^{-1}$ and, together with $\underline{\mu}_{0} \underline{\mathrm{M}}_{\mathrm{sp}}=2.222 \mathrm{~T}$ and the fact ${ }^{15}$ that the direction of - $_{\text {LORENTZ }}+$ E $_{\text {FERMI }}$ is opposite

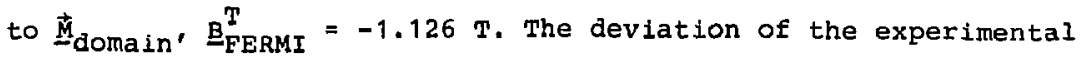
data from this extrapolation is attributed to the occupancy, 
increasing with decreasing temperature, of octahedral sites in addition to tetrahedral sites. The implication that $\mu^{+}$at 0 sites are metastable with respect to T-site occupancy is compatible with (ii). If for an order-of-magnitude estimate we assume that at $T=0$ the probability of occupation 1 s the same for both types of site, we find $\underline{B}_{\text {FERMI }} \approx-1.175 \mathrm{~T}$.

Insertion of $\underline{B}_{\mathrm{FERMI}}^{\mathrm{T}}$ into Eq. (2) gives us, at $\underline{\mathrm{B}}_{\mathrm{appl}}=4.5 \mathrm{~T}$, $\underline{\omega}_{\mu}=28.7 \cdot 10^{8} \mathrm{rad} \mathrm{s} \mathrm{s}^{-1}$, hence from Eq. (3) $\hat{I}_{c}=I_{c}(3.7 \mathrm{~K})=3.5 \cdot 10^{-10} \mathrm{~s}$, and from Eq. (5) $\underline{B}=13 \cdot 10^{-10} \mathrm{~s} \mathrm{~K}$. From Eq. (4) we find $\left|\mathrm{B}_{\mathrm{dip}}\right|=0.34 \mathrm{~T}$. The interpretation of the latter value as $\left|\underline{B}_{\mathrm{dip}}^{\mathrm{T}}\right|$ is consistent with our earlier conclusion ${ }^{5}$ and with footnote ${ }^{6}$.

Two results of our experiments have not yet been discussed:

(a) At short times the majority of the measured relaxation functions deviate strongly :rom a single exponential. At first sight, this effect, which is not due to experimental inadequacies, is very surprising, since under quite general assumptions ${ }^{2,7}$ the longitudinal relaxation function of a spin-1/2 $y$ stem in contact with a heat bath should be exponential. Th. of, rved deviations point strongly towards metastability effec

(b) Below about $5 \mathrm{~K}$ the longitudinal celaxation rates measured at $2 \mathrm{~T}$ deviate strongly from $\Gamma_{1}^{\langle 119\rangle}(\underline{T})$ as deduced above (Fig. 1$)$. Both phenomena are accounted for by the model proposed for the interpretation of $\underline{\omega}_{\mu}^{o}(\underline{T})$. Immediately after their implantation, $\mu^{+}$occupy both $\mathrm{T}$ sites (stable) and o sites (metastable). The long-time longitudinal relaxation is always describable by a single exponential, i.e. by a relaxation rate constant. This constant (plotted in Fig. 1) is given by $\underline{-}_{-1}^{\langle 11\rangle}$ or the transition rate from octahedral to tetrahedral sites (equal to 4y notation of ref.7), depending on which one is smaller. At $\underline{B}_{a p p l}=4.5 \mathrm{~T}$ 
(corresprnding to $\underline{B}_{\mu}=3.37 \mathrm{~T}$ ) this is $\underline{\Gamma}_{1}^{\langle 111\rangle}$ over the entire tempera دre range Investigated, whereas at $\underline{B}_{\mathrm{appl}}=2.0 \mathrm{~T}\left(\underline{B}_{\mu}=0.87 \mathrm{~T}\right)$ a cross-over takes place between $2 \mathrm{~K}$ and $3 \mathrm{k}$. Hence below about $5 \mathrm{~K}$ the $2 \mathrm{~T}$ rates plotted $\mathrm{In} \mathrm{FIg} .1$ are only lower limits to $r_{1}^{<1113}$.

As a result of the present work, the following conclusions may be added to $(1)-(1 i 1)$ and those of ${ }^{2}$ :

(iv) The maximum predicted by (1) at $\underline{\omega}_{\mu} \underline{\tau}_{c}=1$ has been found. From its temperature and height one may conclude that under equilibrlum conditions at low temperatures positive muons in a-Fe occupy tetrahedral and not octahedral interstices. The dipolar field felt by the $\mu^{+}$in tetrahedral interstlces with tetragonal axis parallel to the magnetization is given by ${ }_{-\mathrm{dip}}^{\mathrm{T}}=-0.34 \mathrm{~T}$.

(v) Between $0.85 \mathrm{~K}$ and $12 \mathrm{~K}$ the $\mu^{+}$jump frequency between tetrahedral interstices is proportional to the absolute temperature I; the diffusivity in this range is given by

$$
\underline{D}^{\mu^{+}}(\underline{T})=\underline{a}_{o}^{2} / 72 \underline{T}_{c}=8.7 \cdot 10^{-13} \underline{\underline{I}} \mathrm{~m}^{2} \mathrm{~s}^{-1} \mathrm{~K}^{-1}
$$

$\left(\underline{a}_{0}=2.85 \cdot 10^{-10} \mathrm{~m}=\right.$ edge length of the elementary cube) . The linear temperature variation constitutes strong evidence for Incoherent tunnelling by one-phonon processes.

(vi) Evidence has been found that Immediately after 1 mplantation into $\alpha-F e$ a certain fraction of the $\mu^{+}$occupy metastable sites.

This work was financlally supported by the Bundesminister für Forschung und Technologie, Bonn. Discussions with Dr. L. Schimmele, the technical assistance of $M$. Krenke, and the support of SIN are gratefully acknowledged. 
(a) Alexander von Humboldt scholar 1978-80; permanent address: RIKAGAGKU KENKYUSHO, The Institute of Physical and Chemical Research, HIROSAWA, WAKO-SHI, SAITAMA, JAPAN. (b) permanent address: Hung̣arian Academy of Sciences, Resar ah Institute for Technical Physics, BUDAPEST, HUNGARY.

${ }^{1}$ For up-to-date references see Proc. Yamada Conference on Muon Spin Rotation and Associated Problems, Shimoda, Japan, Hyperf. Int. $17 / 18 / 19(1984)$

${ }^{2} \mathrm{~A}$ Seeger, in: Hydrogen in Metals I, edited by G. Alefeld and J. Völkl (Springer, Berlin-Heldelberg-New York, 1978),p.349 ${ }^{3}$ D. Herlach, H. Metz, A. Seeger, E. Yagi, K.-P. Arnold, M. Gladisch, W. Jacobs, H. Orth, G. zu Putlitz, and J. Vetter, Research Proposal RA-78-02.1, SIN, Villigen, 1978 "Yu. M. Belousov, V.N. Gorelkin, A.L. Mikaélyan, V. Yu. Miloserdin, and V.P. Smilga, Sov. Phys.-Usp. 22, 679 (1979)

${ }^{5}$ E. Yagi, H. Bossy, K.--P. Döring, M. Gladisch, D. Herlach, H. Matsui, H. Orth, G. zu Putlitz, A. Seeger, and J. Vetter, Hyperf. Int. 8,553 (1981)

${ }^{6}$ Throughout this paper, the magnitude $\underline{B}_{d i p}$ of these fields at the $\mu^{+}$sites with tetragonal axes parallel to $\overrightarrow{\mathrm{N}}_{\text {domain }}$ is used as a

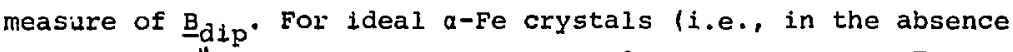
of $\mu^{+}$, the $\underline{B}_{d i p}^{l l}$ values are known as $\underline{B}_{d i p}^{O}=1.86 \mathrm{~T}$ and $\underline{B}^{T}=-0.522 \mathrm{~T}$, respectively. (As a convention ${ }^{7}$ + signs are used for fields parallel or antiparallel to $\overrightarrow{\underline{M}}_{\text {domain }}$. on theoretical grounds, we expect that for occupled sites the absolute magnitudes of the dipolar fields are smaller than but not. radically different from the values just quoted, so that $\left(B_{\text {Ciip }}^{O}\right)^{2} \gg\left(B_{d i p}^{T}\right)^{2}$ should continue to hold.

${ }^{7} A$. Seeger and P. Monachesi, Phil.Mag. B46; 283 (1982)

${ }^{8} \mathrm{H}$, Teichler and A. Seeger, Ptys.Lett. $\underline{82 \mathrm{~A},} 91$ (1981) 
'A. Seeger and C. Schmidt, to be published

${ }^{10} \mathrm{~K}$. Lub.itz and G. Göltz, Appl. Phys. 19, 237 (1979)

${ }^{11} \mathrm{~K}$. Maier and $A$. Seeger, in Electronic Structure and Properties of Hydrogen in Metals, edited by P. Jena and C.B. Satterthwaite (Plenum, New York, 1983), p. 601

${ }^{12} \mathrm{M}$. Krauth, Diploma thesis, Universität Heidelberg, 1983

${ }^{13}$ For further conclusions, see A. Seeger, Hyperf. Int. 17/18/19 (1984) 987

${ }^{14} \mathrm{H}$. Graf, G. Balzer, E. Recknagel, A. Weidingcr, and R.Y. Grynszpan, Phys.Rev.Lett. 44, 1333 (1980)

${ }^{15}$ I.I. Gurevich, A.I. Klimov, V.N. Maľorov, E.A. Melestako, B.A. Nikol'skiY, V.I. Selivanov, and V.A. Suetin, Sov.Phys.JETP 42, 222 (1976)

Figure captions

Elg. 1 Longicudinal $\mu^{+}-$spin relaxation rates in a sphexical a-Fe monocrystal at various applied magnetic fields $\vec{B}_{\text {appl }}$ parailel

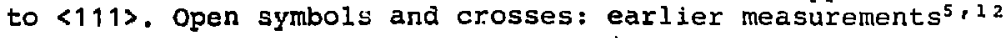
Full symbols: present data. Inset: $\mu^{+}$precession Erequency in demagnetized $\mathrm{Fe}^{14}$. The lines represent fits described in the text. Eig.2 $\left(T \Gamma_{1}\right)^{-1}-T^{-2}$ and $T / I_{1}-T^{2}$ plots of the long-time (t $\geq 60 \mathrm{~ns}$ ) relaxation rates at $\overrightarrow{\mathrm{B}}_{\mathrm{app} 1}=4.5 \mathrm{~T}$ parallel to $<111>$. 


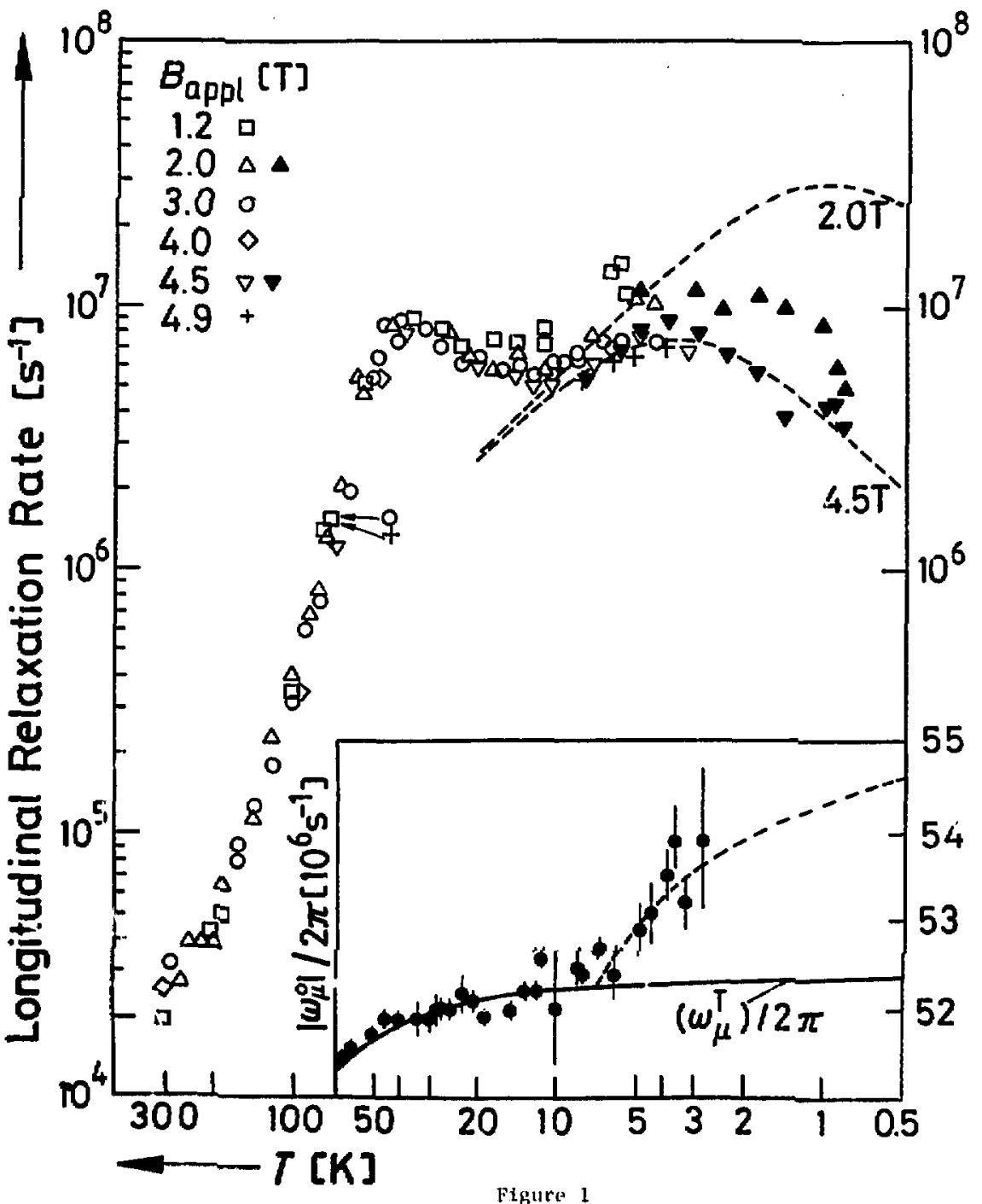




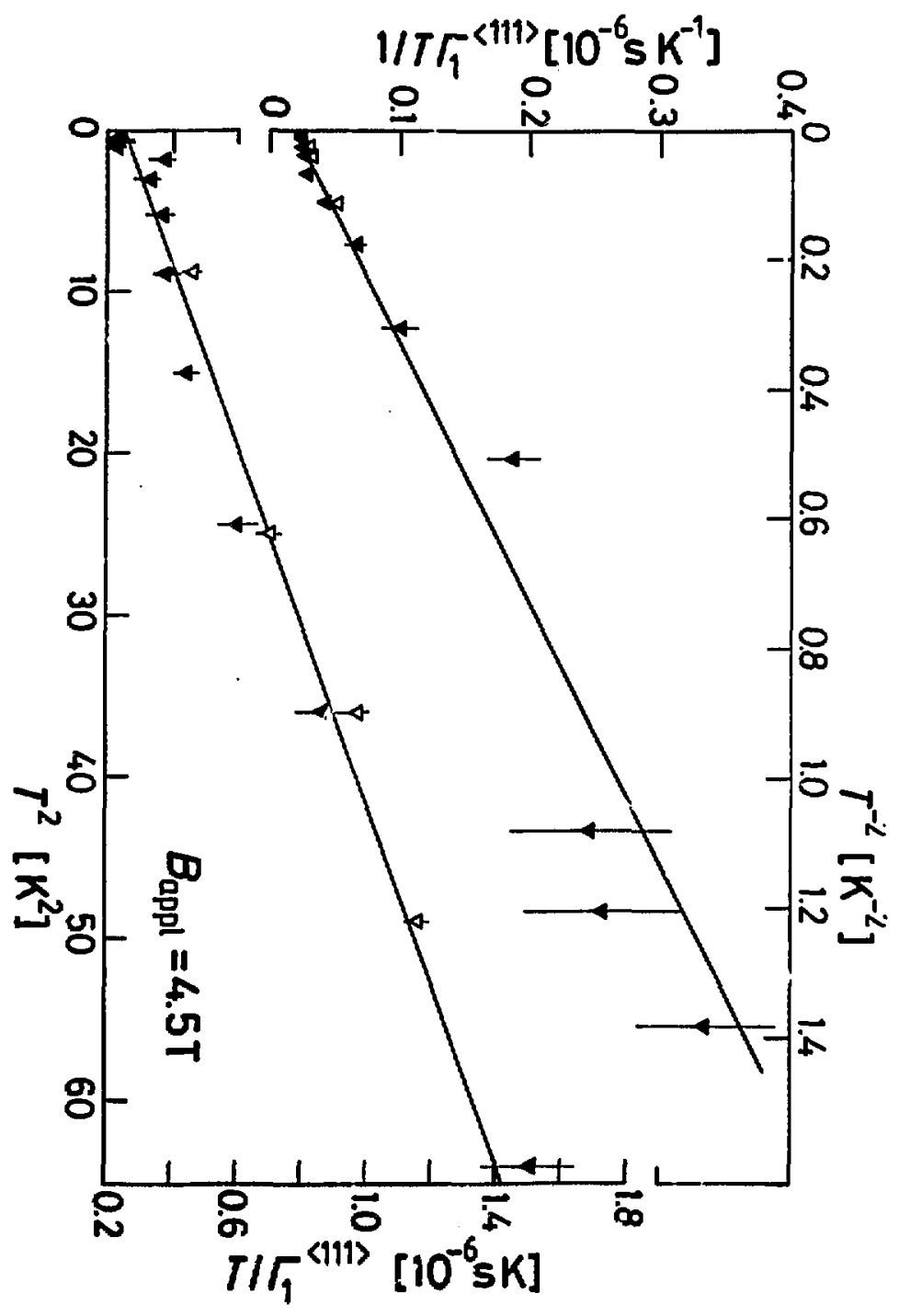

Figure 2 
A COMPARISON OF 'SPIN-GLASS DYNAMICS DETERMINED BY THE MUON-SPIN RELAXATION AND NEUTRON SPIN-ECHO TECHNIQUES

\title{
R. B. Heffaer
}

Lot Alanos National Laboratory, Los Alamos, New Mexico 87545

D. E. MacLaughiln

Dept. of Physics, University of Callfornia, Riverside, Cailfornia 92521

\begin{abstract}
Impurity-spln correlation functions obtalned from muon-spin relaxation and neutron spin-echo experiments are found to agree quantitatively above and below the "glass" tenperature $T_{B}$. The agreement confirms the power-law decay of correlations in time near and below $T_{g}$. Features of the experimental results whlch depend on (quasi)static lmpurity-spin correlations ungese that the satial distribution of thermally-everaged Impurity-spin wagnitudes is quite unifora below $T_{g}$. The etochastic behavior of dipolar fielde at won sites is essentially the same as that of the lopurity spins themselves at all tenperatures.
\end{abstract}




\section{INTRODUCTION}

The dynente behnvior of disordered epln eystens poseesing random exchange is a ubfect of conelderable current interest. In elaple theoretical oodelo for cryetalline (nonrandon) epln eystens, auch ae ordered ferroagnets, it 1 found thet the epin autocorrelation function $s_{\sigma}(t)$ $\langle\vec{\sigma}(t) \cdot \vec{\sigma}(0)\rangle$ for the atochestic tee dependence $\vec{\sigma}(t)$ of the Inpurtty epine decays exponentially in time (outeide of the critical region). I In disordered systens, however, dynamic theories ${ }^{2}$ and elmulations ${ }^{3}$ predict an algebralc (power-1aw) decay In tine. It 10 therefore of Iaterest to pursue experimentally the distinction between dynancs in ordered and disordered eystems.

Recently two relatively new experimental techniques, neutron epin-echo (NSE) scattering and wuon-spin relaxation ( $\mu \mathrm{SR})$, have been used to probe epin dynanice in metullic epin glases. These are dilute magnetic alloys (e.g. AgMn, CuHn, AuFe), which exhibit a characteristic cuep in the ac susceptibility at the glase freezing temperature $T_{q}$. The NSE technique, introduced by Mezel and Murand, 5 measures the epln correlation function $s_{d}(\ddot{q}, t)$ drectly, where $\vec{q}$ is the neutron monent um transfer. The uSR method 6 yielde epin-lestice relaxation rater of locel probes (1mplanted posiIIve auns), which are ensitive to fluctuations of the (dipolar) local cield $\vec{h}(t)$ at wuon sites. Thts yields a broad average over all $\vec{q}$, as in aucleer magnetic resonance (MIR). The time dependence of the Iocal-field correlation function $s_{h}(\vec{q}, t)$, avaraged over all $\vec{q}$, cun be meaured 1ndirecty using $\mu S R$ in longleudinal applied fleld, and ihe result cen then be cospared to $s(\vec{q}, t)$ obtained ueing NSE In zero field. The two cech-

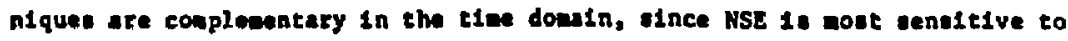


correlation tien in $s_{\sigma}(\vec{q}, t)$ between $10^{-8}$ and $10^{-12}$, whereas $\mu \mathrm{SR}$ is not ceastive to tiace between $10^{-4}$ and $10^{-11}$.

In this paper HSR and WSE aseureants of Inpurity-epin correlation functions in etellic opin glasen are conpred. The results are also compared to dyantcal theories. Prelininary results of this analyols have been previously presented.?

\section{MUON-SPIN RELAXATION DATA}

Recently the longltudian field dependence of the muon spia-lattice

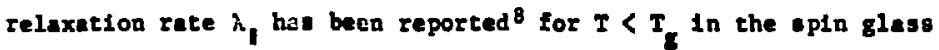

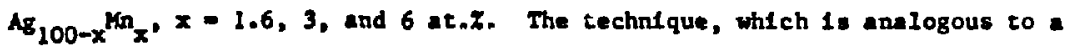
measurenent of $T_{1}$ in MMR, has been deocribed elswere.9010 The measurements were carried out in applied flelds between 0.15 and 5 kOe $\left(g_{B}{ }_{B} \ll\right.$ $k_{B} T$ ) and at temperatures between $0.3 T_{g}$ and $0.92 T_{g}$. It was found that $\lambda_{\|}=$ $K(T) \omega_{p}^{v-1}$, where $\omega_{\mu} 1$ a the Iernor frequency of the nuon and $K(T)$ is a terperature-dependent constant. The neasured valued of $v$ are given in Table I. Comblning these resulte in the temperature range $0.3<T / T_{\mathbf{g}}<0.66$ $y 1$ elds $v=0.54 \pm 0.05$. For $T / T_{g}=0.92$ one obtains $v=0.24 \pm 0.02$.

For wuone at rest in the saple one axy argue on very general grounds 11 that $\lambda_{1}$ 1. proportional to the nolse power $J_{h}\left(\omega_{\mu}\right)$ in the fluctuating fleld at the wuon Latwor frequency $\omega_{\mu^{\prime}}$ assubting that the applied field does not appraciably affect the Iepurity-epin dynanice. The eseunption of stationary mone 1s known to be true at the tesperaturea of Interest. 12 The assumption of t1eld-independent dynavice 1s reanonable for sall fields and tenperetures vell belou $T_{z}$, and 10 borne out by MMR measurenente In CuMn13

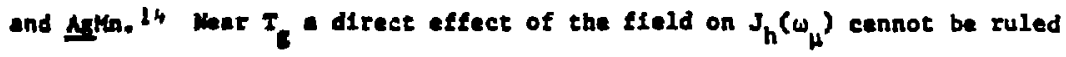
out, as discuesed blow. 
III. NEUTRON SPIN-ECHO DATA

FIgures 1 and 2 give NSTE reeulte 5.15 for $s_{\sigma}(\vec{q}, t)$ In $\mathrm{Cu}_{99^{\mathrm{Mn}}}, I_{t}=10.1$ $K(F I g, 1)$, and $\mathrm{Cu}_{95} \mathrm{Iin}_{5}, \mathrm{~T}_{\mathrm{C}}=27.5 \mathrm{~K}(\mathrm{FIg}, 2)$. The $\mathrm{s}_{\sigma}(\vec{q}, t)$ data were found to be essentially Independent of $\vec{q}$ over the meesured range $60.045 A^{-1}<q<$ $0.36 A^{-1}$, and we ehall wite $\xi(t) \equiv s_{\sigma}(q, t)$ henceforth. The form of $\xi(t)$ was found to be exponentsal for $T \gg I_{g}$, but a long-tine tall developed near and below $T_{8}$. The curves In FIgs. 1 and 2 are nodel fits a discussed below.

IV. COMPARISON OF NSE AND HSR: $T<T_{g}$

In order to compere the NSE aeasurements of $g(t)$ to $\mu S R$ spin-1attice relaxation measurements, we take a smplified form for the sotal spectral density

$$
J_{h}(\omega)=A \delta(w)+\mathbf{J}_{h}(w)
$$

of muon local-field fluctuations. Here the first term 1 s reloced to the quasistatic response of the system, and the second tern gives the dynamic fluctuation density. In real eystems the first term may possess a nonzero (but smal1) width which cannot be detected using finfte probe fraquencles. Taking the Fourier tranaform of $J_{h}(w)$ ylelds the autocorrelation function $s_{h}(t)$ of the nuon locsl field

$$
S_{h}(t)=\int_{-\infty}^{\infty} d \omega J_{h}(\omega) e^{1 \omega t}-\Lambda+(1-A) F(t)
$$

where $r(0)-1, r(-)=0$, wo chat $A=s_{h}(-)$ 
In real epin clese an upper liat to the epectrue of flucturtion frequenclea exdetw. We take thd upper linit to be of the order of an ex-

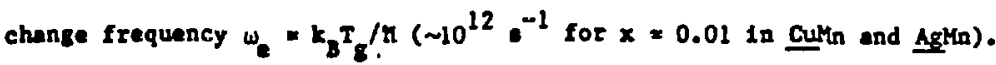
The uSR data 1aply a power-law form $J_{h}(\omega)=\omega^{v-1}$, so that

$$
F(t)=\left(\omega_{e} t\right)^{-v}, \omega_{e} t \gg 1 \text {. }
$$

Ualng the form of the correlation function given by Eqs. (1) and (2), and the time dependence Implled by the $\mu S R$ results [Eq. (3)], values of $s_{h}(t)$ are obtained and compared wth the measured $\xi(t)$ from NSE. This procedure assumes that the form of the correlation function is the sage for fluctuations of the Impurity opins and the muon local fielda; evidence for the valldity of this assumption will be given below in Sect. $v$. MSE measurenents Ia Culin wil be compared to pSR weasuremente in Aging and AuFe, as well as Culfn, and $1 \mathrm{t}$ nust also be sssumed that neasured frequencies, rates, Inverse times, ecc., can be scaled by the respective glass temperatures of each sample.

Least-squares fits of the NSE data have been perforned using Eqs. (2) and (3), wth free paraneters $A, w_{e}$, and $v$. In general the NSE data are not preclse enough to determine all of these paraweters accurately, and it must be decided inatead whether a physically reasonable set of parameters are consistent with both ${ }_{2} S R$ and NSE experimental resulta. The curves in Figw. 1 and 2 gtve typical fits. It can be ween that the form of Eqa. (2) and (3) represent the NSE daca well, using the estimates $\omega_{e}=10^{12} \mathrm{~s}^{-1}$ for

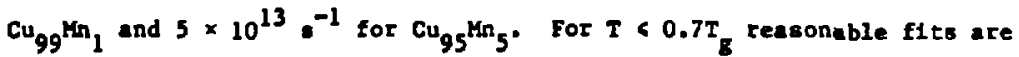
obtalned wth $v=1 / 2$, which 1s the value obtalned from the HSR data, 8 although the neutron data do not determine $v$ well, One can see from Figs. $1 \& 2$ 
that at $T / T_{z}=0.7$, for exasple, $A(T) 10-0.6$ for $\mathrm{Cu}_{95} \mathrm{Fin}_{\mathrm{s}}$ and -0.4 for $\mathrm{Cu}_{99} \mathrm{Mn}_{1}$. The NSE date therefore do not obey the expected callng $1 \mathrm{aw}$, ac noted previously. 15

Below $T_{g}$ the whort-tine behavior of the longltudinal nuon relaxation function $G_{1}(t)$ provides a direct experinental Infortacion on the distribution of static wuon locel fielde, and to therefore related to the long-tine behavior of $s_{h}(t)$ [1.e. the value of A In Eq. (2)]. Keasurenents of $G_{1}(t)$ In AgMn, 10 AuFe, 16 and CuMbn 17 for Impurity conceatrations -1 at. $z$ have determined the width $a(T)$ of the Lorentzian diotribution of stacic local flelds in each of these aystewe. Below $T_{g}$ (T) Increases with decreasing temperacure from $a\left(T_{g}\right) \sim 0$ to the frozen-spin value a(0) for $T<T_{g}$. Figure 3 gives the dependence of $[a(T) / a(0)]^{2}$ on reduced temperature $T / T_{g}$ for the above systems. Within about $20 \%$ uncertalaty the data sale well. We note that for $T / T_{g}-0.7$ the value of $[a(T) / z(0)]^{2}=0.4$ agrees well with the NSE value of $A\left(T / T_{g}-0.7\right)$ for $\mathrm{Cu}_{99} \mathrm{Mn}_{1}(F 1 g .1)$, but is signtficant1y smaller than $\mathrm{A}\left(\mathrm{T} / \mathrm{T}_{\mathrm{g}}-0.7\right)=0.6$ for $\mathrm{Cu}_{95} \mathrm{Mn}_{5}(\mathrm{~F} 1 \mathrm{~g} .2)$.

Walstedt and Walker ${ }^{18}$ have shown that under some general assumptions $\left(r^{-3}\right.$ Interaction, low impuxity concentration) the linewidth $a(T)$ of a probe spin (muon, nucleus) in a magnetic alloy is related to the impurity spin magnitude by

$$
a(T)=\left\langle\left|\left\langle\vec{\sigma}_{1}\right\rangle_{T}\right|\right\rangle_{1}
$$

Here $\zeta\rangle_{I}$ and \langle\rangle$_{1}$ signtfy therwal and spatial averages, respectively. Equation (4) is valid in the rapid-fiuctuation $11 \mathrm{mlt}, 1$,e. for 1mpurdtyepin thermal fluctuation rates wuch larger than the resultant probe-spin IInewdth a(T), 10 whtch seems to be well establiohed for metallic epin 
glases. $10 \cdot 16: 17$ Koreover $A(T)$, defined In $\mathrm{Eq} .(2)$, Is given by

$$
A(T)=\left\langle\left\langle\left.\left\langle\vec{\sigma}_{1}\right\rangle_{T}\right|^{2}\right\rangle_{1} / s^{2}\right.
$$

Comparison of $a(T)(H S R)$ and $A(T)$ (ASE) then ylelds Information on the epatial distribution of spin magnitudes $\left\langle\sigma_{1}\right\rangle_{T}$, as can be established by conalderlng two extreme caes: 16

I. The $\left\langle\vec{\sigma}_{1}\right\rangle_{T}$ are all the vame. Then

$$
\left\langle\left|\left\langle{\overrightarrow{\sigma_{1}}}_{1}\right\rangle_{T}\right|^{n_{1}}\right\rangle_{1}=\left|\left\langle{\overrightarrow{\sigma_{1}}}_{1}\right\rangle_{T}\right|^{n}
$$

for any $\underline{n}$, and

$$
A(T)=[a(T) / a(0)]^{2}
$$

As noted above, this 18 consistent wth $\mu$ SR data for $x=1$ at. $z$ and the NSE results for $\mathrm{Cu}_{99} \mathrm{Mn}_{1}$ at $\mathrm{T} / \mathrm{T}_{\mathrm{g}}=0.7$.

B. A Eraction $\underline{f}$ of the spins are saticated $\left(\left|\left\langle\vec{\sigma}_{1}\right\rangle_{I}\right|-s\right)$, and the remaining fraction is "free" $\left(\left\langle\left\langle\sigma_{1}^{-\infty}\right\rangle_{T}=0\right)\right.$. Then

$$
\left\langle\left|\left\langle\vec{\sigma}_{1}\right\rangle_{T}\right|^{n}\right\rangle_{1}=I S^{n}
$$

for any $\underline{n}$, and

$$
A(T)=a(T) / a(0)-f
$$

The compar1son between $\mu S R$ and NSE date ouggesta, therefore, that the Im- 
purity oplas are dietributed in direction but not in agaicude below $\mathrm{T}_{\mathrm{g}}$. Thi In tirn ceens consistent with a "percoletion" plcture of the tranoltion at $T_{g}$ only if the "Infinite cluster", the formation of which 1 e taken to define $I_{g}$, quickly eacompasses essentially all Inpurity spins as the temperature 1 s lowered below $T_{g}$. NSE resulte at nore temperatures in sawples with $x=0.01$ would be uneful in extending this conclusion, as well as USR data at higher concentrations.

IV. COMPARISON OF NSE AND $\mu$ SR: $T>\mathbf{T g}$

The form of $\xi(t)$ as wessured by NSE changen for $T>T_{g}$, and eventually cends toward an exponential for $T \gg T_{g^{*}}$. Spin probes in rapidly-fluctuatigg local fields are not sensitive to the functional form of $s_{h}(t), 11$ but yield an effective correlation time $t$ defined by

$$
\tau \equiv \int_{0}^{\infty}\left[s_{h}(t) / s_{h}(0)\right] d t
$$

Values of $t$ have been obtained for AgMn, 10 AuFe, 16 and CuMn' ${ }^{18}$ from zerofleld 1 SR measurements. Figure 4 1s a scaled plot of $\tau T_{g}$ vs. T/ $T_{g}$, reproduced from Ref. 10.

Meze1 15 has obtalned good flts of $\xi(t)$ data obtained from NSE in $\mathrm{Cu}_{95} \mathrm{Mn}_{5}$ to the form

$$
E(t)=\frac{1}{E_{1}} \int_{0}^{E_{H}} \exp \left[-t /\left(\tau_{0} e^{E / T}\right)\right] d E
$$

which was motivated by considerations of distributione of barrler hefghts 
for actlvated hopplng. (Its val1dity is not necessarly a demonstration of the 1mportance of euch barriers, however.) A good fit was obtained for $\mathrm{F}_{M}$ $-300 \mathrm{~K}$ and $\tau_{0}=6 \times 10^{-14} \mathrm{c}$. Scaled values of these parameters, together wth Eq8. (10) and (11), yield the curve In Fig. 4. There 1s good agreement between the HSR and NSE data, with no adjustable paraweters. This confirms the simflarity between mon local-field and 1mpurity-8pin stochast1c behavior assumed above, over a wde range of values of $\tau$.

\section{v. CONCLUSIONS}

Muon-spin relaxation and neutron epin-echo measurements of local-field and impurity-spin correlation functions $\xi(t)$ in metali1e spin glasses give consistent resulte for temperatures above and below $\mathrm{T}_{\mathrm{g}}$. The time-dependent component of $\xi(t)$ decays as $t^{-v}, v=1 / 2$, below $T_{g^{*}}$ This behavior $1 \mathrm{~s}$ conGistent wth recent mean-rifeld theorles of 5 pin-glass dynamics, ${ }^{2}$ as well as wth earlier Monte-Carlo simulations. ${ }^{3}$

For CuMn, AgMn, and AuFe spin glasses with simflar values of $T_{g}( \pm 30 \%)$, scaled static $\mu S R$ linewldhs agree well below $\mathrm{T}_{\mathrm{g}}$. Furtherwore, the relation $A(T)=[a(T) / a(0)]^{2}$, expected for a uniform distribution of 1mpur1ty-spin moment magnitudes, is obeyed for 1mpurity concentrations $x=1$ at.7. This agreement breaks down, however, for comparison with NSE data on $\mathrm{Cu}_{95} \mathrm{Kn}_{5}$, where, as noted by Mezel and Muran, 5.15 the larger value of $\mathrm{A}(\mathrm{T})$ Implies an Increase in static spectral density wth increasing Impurity concentration,

Near the glass temperature the relation $\xi(t)=t^{-\nu}$ still seems to hold, but $v$ deviates from $1 / 2$ for the AgMn $\mu$ SR and $\mathrm{Cu}_{95} \mathrm{Mn}_{5}$ NSE dita. Curiously, the $\mathrm{Cu}_{99} \mathrm{Mn}_{1}$ NSE data still yield $v=1 / 2$ at $T / T_{g}=1.1$. $\mu S R$ experiments are not reliable 1ndicators of the value of $v$ near $T_{g}$, however, since 
$J_{h}\left(\omega_{\mu}\right)$ way depend on field directly as well as via the won warmor frequency. Above $T_{g}$, where $\xi(t)$ tends toward an exponential form, $\mu S R$ and NSE data yield consistent correlation tines as ween in Fig. 4. NSE and HSR weaurenents are therefore in reasonable quantitative agreewent In wetalile opin glases. This Impliso (1) that the $q$ independence of $S_{\sigma}(q, t)$, obtaloed from NSE measurementa for a restricted range of q, apparently holda over the wider range of $\vec{q}$ values laportant for $\mu S R$; (2) $A$ consiatent time dependence of $s_{\sigma}(\vec{q}, t)$ ls obtained for measuring tiaes $610^{-9}$ (NSE) and $>10^{-9} *(\mu S R)$; and (3) perhaps most 1afortantly, wore confidence in the reliablity of the experimental determinations 18 warranted than could be given to elther technique taken by 1 tself. Acknowledgerents

We are grateful for useful discusstons with D. L. Huber and R. E. Walstejt, and to M. Leo: for collaboration in a preliminary version of this report. Th1s work was performed under the auspices of the U. S. Department of Energy, and was also supported by the U. S. Natlonal Sclence Foundation (Grant No. DMR 81-15543). 


\section{REFERENCES}

1. S.-K. Ma, Modern Theory of Critical Phenomena (Benjamin, Reading, Massachusetts, 1976).

2. H. Sompolinsky and A. 21ppel1us, Phys. Rev, B 25, 6860 (1982).

3. S. K1rkpatrick and D. Sherrington, Phys. Rev. B 17, 4304 (1978).

4. v. Kannella and J. A. Mydosh, Phys. Rev. B 6, 4220 (1972).

5. F. Meze1 and A. P. Murani, J. Magn. Magn. Mat. 14, 211 (1979); A. P. Murant, F. Meze1, and J. L. Tholence, Physica 108B, 1283 (1981).

6. A. Schenck, in Nuclear and Particle Physics at Intermediate Energies, edited by J. B. Warren (Plenum Press, New York, 1976), p. 159.

7. R. H. Heffner, M. Leon, and D. E. MacLaughlin, in Proc. Yamada Conf. on Muon Spln Rotation and Related Problems, Shimoda, Japan, 1983 (Hyperf. Int., in press).

8. D. E. MacLaughlin, L. C. Gupta, D. W. Cooke, R. H. Heffner, M. Leon, and M. E. Schillac1, Phys. Rev. Lett. $\underline{51}, 927$ (1983).

9. Y. J. Uemura, K. Nishi yama, T. Yamazaki, and R. Nakaf, Solid state Commun. 39, 461 (1981).

10. R. H. Heffner, M. Leon, M. E. Schillac1, D. E. MacLaughlin, and S. A. Dodds, J. Appl. Phys, 53, 2174 (1982).

11. A. Abragau, Princ1ples of Nuclear Magnetism (Clarendon Press, Oxford, 1961).

12. R. H. Heffner, Hyperf. Int. 8, 655 (1981); M. E. Schillaci, C. Boekena, R. H. Heffner, R. L. Hutson, M. Leon, C. E. Olsen, S. A. Dodds, D. E. MacLaughlin, and P. M. R1chards, In Proc. Int. Symposium on the Electronic Structure and Properties of hydrogen in Metals, ediced by P. Jena and C. B. Satterthwalte (Plenum Press, New York, 1983), P. 
139.

13. H. Alloul, S. Murayama, and M. Chapellier, J. Magn, lagn. Mat. 31-34, 1353 (1983).

14. H. Alloul, in Proc. Heldelberg Conf. on Spin Glasses, edited by J. L. van Hemen and I. Morgenstern (Lecture Notes 1n Physics, SpringerVerlog, Berlin, 1983), p. 18; H. Alloul, private communtcation.

15. F. Meze1, J. Appl, Phys. 53, 7654 (1982).

16. Y. J. Uemura and T. Yamazakt, J. Magn, Magn. Mat. 31-34, 1359 (1983).

17. Y. J. Uemura, I. Yamazaky, D. R. Harshman, M. Semba, J. H. Brewer, E. Ansaldo, and R. Keltel, In Proc. Yamada Conf. on Muon Spln Rotation and Related Problems, Shimoda, Japan, 1983 (Hyperf. Int., 1n press).

18. R. E. Walstedt and L. R. Walker, Phys. Rev. B 9, 4857 (1974).

19. Y. J. Uemura, T. Yamazak1, R. S. Hayano, R. Naka1, and C. Y. Huang, Phys. Rev. Lett. 45, 583 (1980). 
TABLE I. Values of the power-law exponent $v$ of the nolse spectrum below $\mathrm{T}_{\mathrm{g}}$ in $\mathrm{Ag} 100-\mathrm{Mn}_{\mathrm{x}}$ :p1n glasses, $1.6<x<6$ at. $x$, obtalned from ISR measurements as described in the text. Data from Ref. 8.

\begin{tabular}{cc}
\hline $\mathbf{T} / \mathbf{T}_{\mathbf{g}}$ & $v$ \\
\hline 0.30 & $0.59 \pm 0.15$ \\
0.46 & $0.51 \pm 0.07$ \\
0.66 & $0.55 \pm 0.07$ \\
0.92 & $0.24 \pm 0.02$ \\
\hline
\end{tabular}




\section{FIGURE CAPTIONS}

FIC. 1. Impurity-spin correlation functions $\xi(t)$ obtalned from neutron epla-echo measurements (Ref. 15) for $T / T_{g}<1.1$ in epin-glass $\mathrm{Cu}_{99} \mathrm{kng}_{1}$. The curves are fite to the functional form of Eqs. (2) and (3), wth parameters as indicated. The dotted lines indicate that Eq. (2) is rigorously correct for $\left(\omega_{e} t\right) \gg 1$. The numbers in parentheses are the reduced temperatures $\mathrm{T} / \mathrm{Tg}$.

FIG. 2. Correlation functions as in FIg. 1, for $\mathrm{Cu}_{95} \mathrm{Mng}_{5}$ (Ref. 5).

FIG. 3. Dependence of the square of the reduced muon local-field distribution widh $[a(T) / a(O)]^{2}$ on reduced temperature $T / T_{g}$ in three metallic spin-glass systems.

FIG. 4. Dependence of the scaled effective correlation time $T T_{g}$ on reduced cemperature $T / T_{g}>1$ In three metallic spin-glass systems. Daca. polnts: results of $\mu S R$ measurements. Curve: fit of Eq. (11) to NSE data for $\mathrm{Cu}_{95} \mathrm{Kn}_{5}$. 


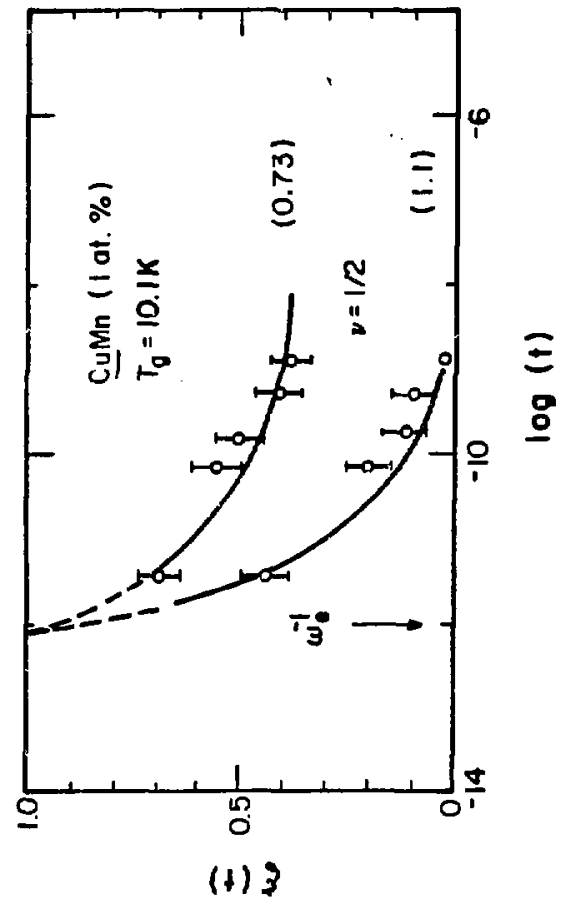

Figure 1 


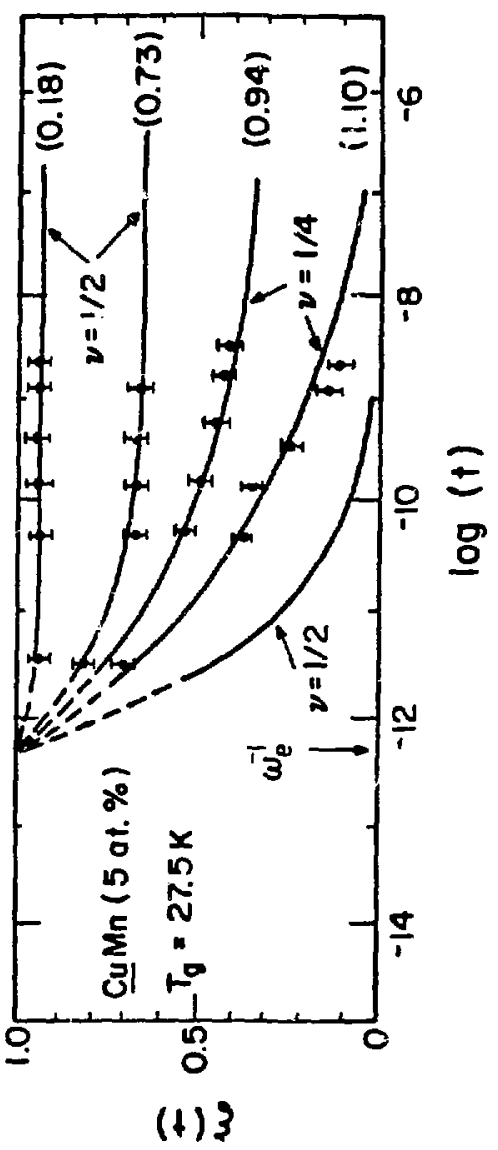

Figure 2 


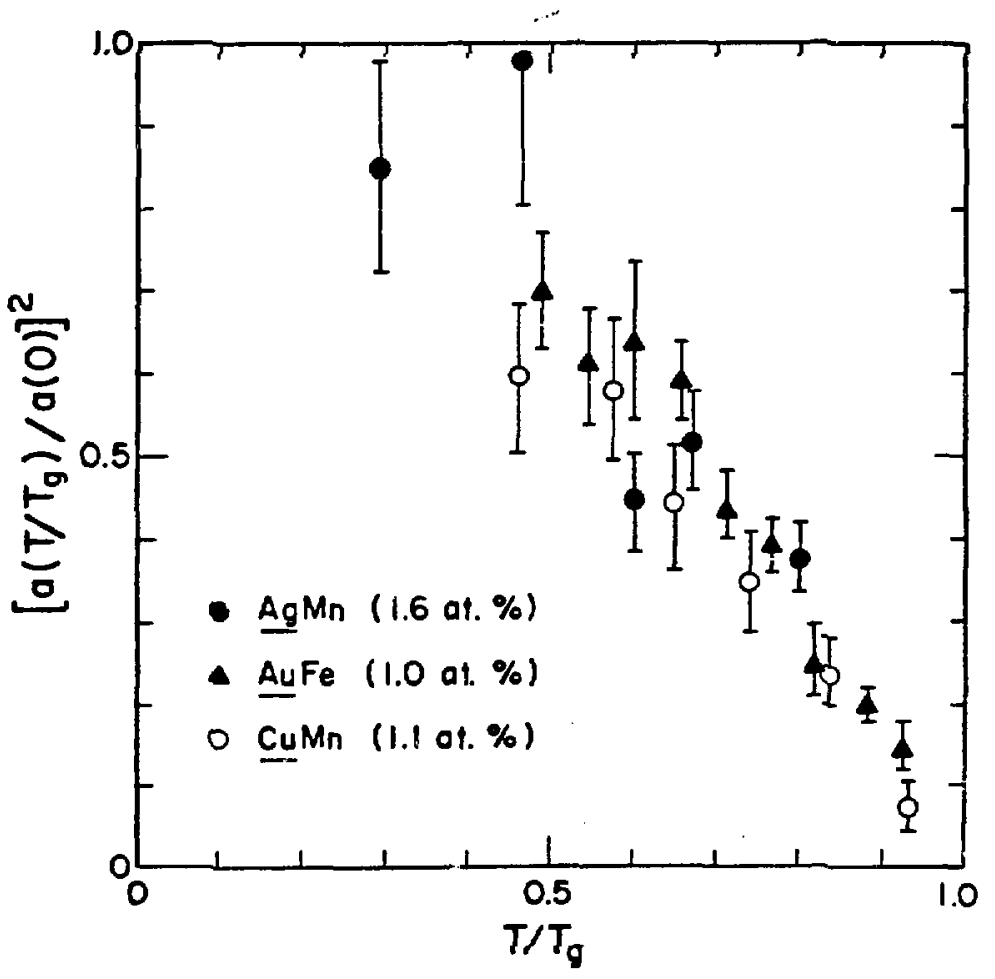

Figure 3 


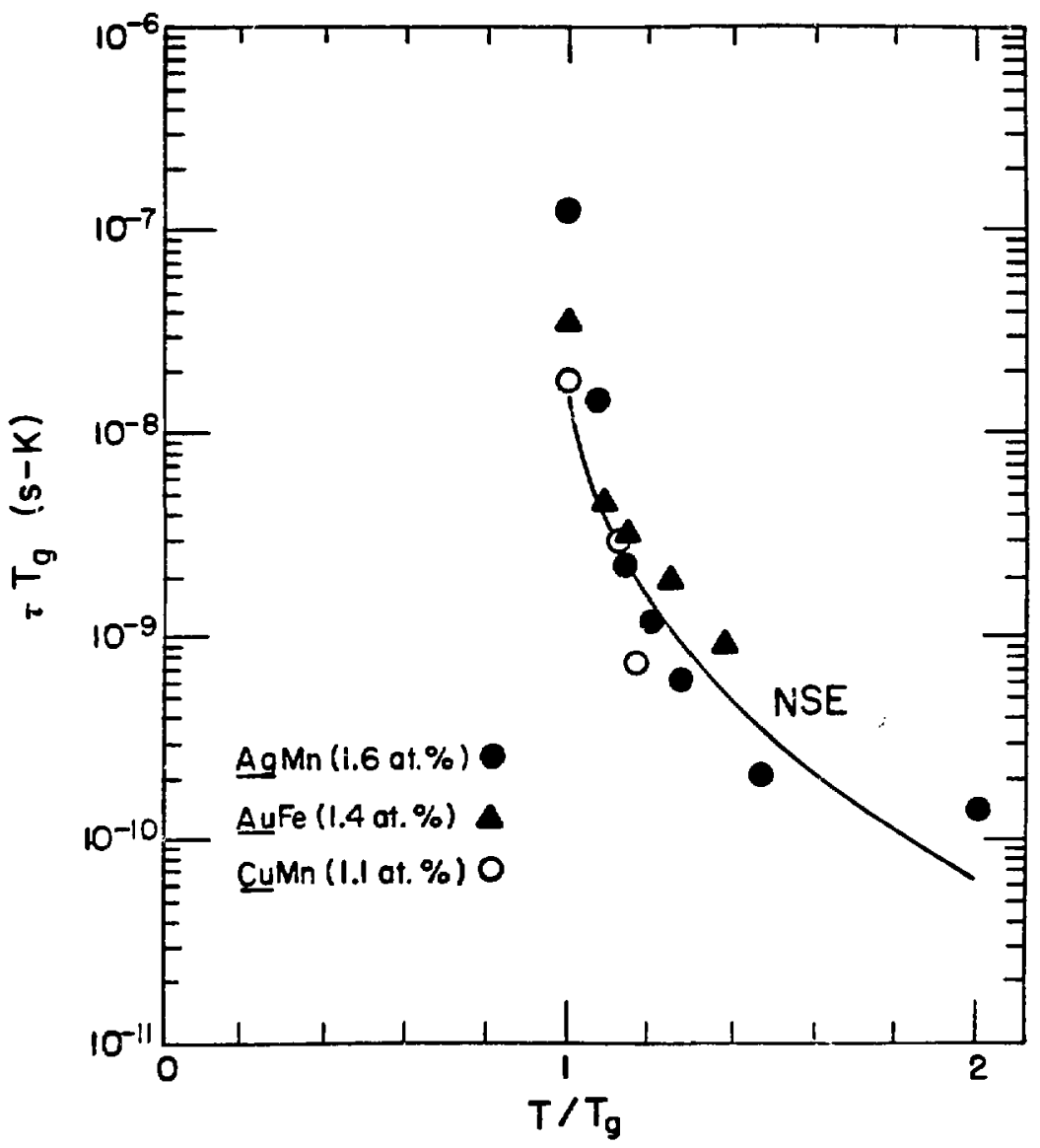

Figure 4 
EFFECTE OF SUPERCONDUCTIVITY ON RARE-EARTH ION DYNAMICS IN

$$
\left(\mathrm{Ho}_{x} \mathrm{Lu}_{1-\mathrm{x}}\right) \mathrm{Rh}_{4} \mathrm{~B}_{4} \text { * }
$$

R. H. Heffner, D. W. Cooke, R. L. Hitson, M. Leon, M. E. Sch1llac1, J. L. Smith, and A. Yaouanc ${ }^{+}$

Los Alamos National Laboratory, Los Alamos, New Mexico 87545

$$
\text { S. A. Dodds }
$$

Rice University, Houston, Texas 7725 ,

L. C. Gupta ${ }^{\neq}$and D. E. MacLaughilin

University of Calffornia, Riverside, Calffornia 92521

PACS numbers: 74.90.+n, 75.30.Hx, 76.90.td

\begin{abstract}
Zero-field muon spin-lattice relaxation rates have been measured In magnetically diluted $\mathrm{Ho}_{x} \mathrm{Lu}_{1-x \mathrm{Rh}_{4} \mathrm{~B}_{4}}$ ternary compounds, primar$11 \mathrm{y}$ for $\mathrm{x}=0.02$. For temperatures below $\sim 11 \mathrm{~K}$ a characteristic two-component structure of the muon depolarization function is observed, which is consistent with slow (quasistatic) $\mathrm{Ho}^{3+}$-moment fluctuations. We have reported simliar behavior for $x=0.7$, which indicates that the slow fluctuations are due to crystalfield 1solation of the $\mathrm{Ho}^{3+}$ ground state. The observed relaxation rates in this temperature regime depend little on $x$, which is consistent with conduction-electron (Korringa) exchange scattering as the dominant mechanism for the fluctuations. The obBerved temperature dependence of muon spin-lattice relaxation in the superconducting state is not presently understood.
\end{abstract}




\section{INTRODUCTION}

The study of the interplay of magnetism and superconductivity, a topic of Interest fox some time in solid-state physics, 1 has recelved renewed interest following the discovery by Mathias and coworkera ${ }^{2}$ of rare-earth (RE) ternary and pseudoternary compounds which exhibit both magnetfc and superconducting phase transitions. A particular class of these compounds, the rare-earth rhodium borides $\left(\operatorname{RERh}_{4} \mathrm{~B}_{4}\right)$, has been extensively studied, ${ }^{3}$ and the ferromagnetic, superconducting, and paramagnetic ohase boundaries for these systems are by now well known. 3,4

In previous publications 5,6 we have reported positive muon $\left(\mu^{+}\right)$spin relaxation ( $\mu \mathrm{SR}$ ) measurements in the compounds $\left(\mathrm{Ho}_{\mathrm{x}} \mathrm{Lu}_{1-\mathrm{x}}\right) \mathrm{Rh}_{4} \mathrm{~B}_{4}, \mathrm{x}=0,0.7$, and 1.0 , and in $\operatorname{GdRh}_{4^{3}} \mathrm{~B}^{-}$A comparison of the $\mu \mathrm{SR}$ measurements in the Hoand Gd-based compounds has established that the $\mathrm{Ho}^{3+}$-moment dynamics are strongly influenced by the crystal-fleld level splittings. 6 The most striking result, however, is a sharp shoulder in the temperature dependence of the $\mu^{+}$spin-lattice relaxation rate at the superconducting transition temperature $T_{s}=7.7 \mathrm{k}$ for $\mathrm{x}=0.7$. In order to investigate further the effects of superconductivicy on the $\mathrm{Ho}^{3+}$-moment dynamics, we have undertaken new measukements for systems w1th $x=0.02$ and 0.35 . Here we report primarily zero-field measurements in $\left(\mathrm{Ho}_{0} .02^{\mathrm{Lu}_{0}} ._{98}\right) \mathrm{Rh}_{4} \mathrm{~B}_{4}$, which becomes superconducting at $T_{s}=11.3 \mathrm{~K}$.

\section{EXPERIMENT AND DATA ANALYSIS}

The experlments were performed at the clinton P. Anderson Meson Physics Facility (LAMPF) using the zero-field, time-differential $\mu$ SR technique. ${ }^{7}$ Sample temperatures were varled between $3 \mathrm{~K}$ and room temperature, 
and were regulated to within $0.1 \mathrm{~K}$. Sample preparation 1 s described in $\operatorname{Ref.5.}$

Three distinct forms for the normalized muon spln relaxation function $G_{z}(t)$ were observed $1 n$ different temperature reglmes. These are plotted in F18. 1 .

A. $T=50 \mathrm{~K}$

At $50 \mathrm{~K} \mathrm{G}_{z}(t)$ was found to be well described by the static zero-field relaxation function derived by kubo and Toyabe, ${ }^{8}$ which is appropriate for a time-independent Gaussian distribution of local magnetic fields at $\mu^{+}$ sites. The measured width of the fleid distribution, about 40 , indicates that the $\mu^{+}$spin relaxation is caused primarily by $\mu^{i}$ precession about the host nuclear dipolar fields. The ret of the long-time portion of $G_{z}(t)$ to $1 / 3$ indicates that the local fle.s is effectively random in direction and static in nature. ${ }^{8}$ Furthermore, the Ho ${ }^{3+}$ spins must be fluctuating so rapidly at $50 \mathrm{~K}$ that they produce no relaxation of the $\mu^{+}$polarization.

B. $15 \mathrm{~K}<\mathrm{T}<50 \mathrm{~K}$

As the temperature is lowered the form of $G_{2}(t)$ changes, until at $15 \mathrm{~K}$ the relaxation function cau be approximated by a simple exponential $G_{z}(t)=\exp \left(-\lambda_{1} t\right)(F 1 g .1)$, where $\lambda_{1}$ is the $\mu^{+}$spt -lattice relaxation rate. The dominant $\mu^{+}$relaxation mechanism at $15 \mathrm{~K}$ is therefore fluctuations of the local field $h_{L}(t)$ produced by the fluctuating Ho ${ }^{3+}$ moments, is discussed below. In the temperature region $15 \mathrm{~K}<T<50 \mathrm{~K} \mathrm{G}_{\mathbf{z}}(\mathrm{t})$ was taken to be the product of an exponential and the Kubo-Toyabe function, because the nuclear and $\mathrm{Ho}^{3+}$ broadenting mechanisus are Independent of each other.

In principle the local-moment contribution to $G_{z}(t)$ in a dilute magnetic alloy at high temperatures should be approximated by the "root exponent1al" $G_{z}(t)=\exp \left[-(\lambda t)^{1 / 2}\right] .9,10$ In practice the presence of the Kubo- 
Toyabe function made determination of the Ho ${ }^{3+}$ contribution to $G_{2}(t)$ uncertaln at high temperatures. The nuclear broadenting will be quenched $1-a$ modest ( 100 Oe) longltuitinal fleld, however, 8 and future experiments in such a fleld will determine the form of the Ho ${ }^{3+}$ contribution more prec1sely.

C. $T<11 \mathrm{~K}$

At $T<11 \mathrm{~K}$ the form of the relaxation function again changes, exh1biting the two-component form shown in Fig. 1 for $T=6 \mathrm{~K}$. These data were fit with the function

$$
G_{z}(t)=[1-a] \exp (-\sigma t)+a \exp \left(-\lambda_{2} t\right)
$$

with $\sigma \gg \lambda_{2}$. One finds that

1) O Is essentially Independent of temperature, with an average value of $11.5 \pm 0.6 \mu \mathrm{s}^{-1}$, or $\sigma / \gamma_{\mu}=130 \mathrm{Oe}\left(\gamma_{\mu}=8.51 \mathrm{Oe}^{-1} \mu \mathrm{s}^{-1}\right.$ is the muon gyromagnetic ratio);

11) a 1s about 0.6 at $T=11 \mathrm{~K}$, and falls to $1 / 3$ for $T<8 \mathrm{~K}$; and

111) $\lambda_{2}$ falls exponentially whth decreasing temperature below $T_{S}$, with en activation energy $\Delta=9.8 \pm 1.3 \mathrm{~K}(\mathrm{~F} \pm \mathrm{g} \cdot 2)$.

From these Iesults we conclude that the stochastic motion of the $\mathrm{Ho}^{3+}$ moments becomes quesistatic below $\sim 11 \mathrm{~K}$. The value of $1 / 3$ for a at low temperatures is Itself Indicative of a random, quasistatic fleld distribution. 8 Furthermore, the temperature 1ndependence of $\sigma$ is evidence that the local fleld $h_{L}(t)$ reorients completely ${ }^{7-9}$ in a time $\tau_{m}$ such that $\sigma \tau_{m} \gg 1$, whence $\lambda_{2}-2 /\left(3 \tau_{m}\right)$. A two-component form for $G_{2}(t)$ can also be due to $11-$ mited-amplitude fluctuations of $h_{L}(t)$ around a static or quasistatic average value, 10 but in such a case $\sigma$ increases markedly with decreasing tem- 
perature as the fluctuation amplitude decreases. This is not observed in

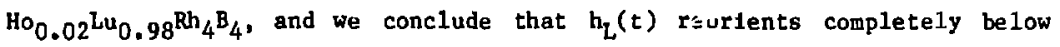
8-10 K.

We have estlmated the value of $\sigma$ using the formalism of Walstedt and Walker ${ }^{11}$ for dipolar coupling between $\mu^{+}$spins and dilute impurity magnet1c moments. (The $\mu^{+}$hyperfine coupling to the conduction electrons is small in metals, 12 and the dipolar coupling dominates indirect mechanisms.) This calculation requires the spatial average $\langle|\mu|\rangle$ of the $\mathrm{Ho}^{3+}$ moment. As discussed below, crystalline-electric-field (CEF) splitting of the Ho ${ }^{3+}{ }^{5} I_{8}$ multiplet produces a two-level Ising-like ground state 5,6 with a magnetic dipole moment of $\sim 10 \mu_{B} \cdot 13$ For randomly-oriented Ho moments this calculation yields $\sigma=6.7 \mu \mathrm{s}^{-1}$, or $\sigma / \gamma_{\mu}=80 \mathrm{0e}$. Th1s Indicates that $\mathrm{H}^{3+} \mathrm{dI}-$ polar fields contribute significantly to the coupling with $\mu^{+}$spins, and is consistent with Identification of the two-component structure of $G_{z}(t)$ with quasistatic inhomogeneous broadening.

\section{DISCUSSION}

Identification of the mechanisms responsible for Ho ${ }^{3+}$-moment relaxation is of considerable 1nterest. Two basic processes, Korringa scattering of conduction electrons ${ }^{14}$ and 1ndirect exchange (RKKY) coupling between $\mathrm{Ho}^{3+}$ spins, 15 should be 1mportant in dilute magnetic alloys. For the Korringa mechanism the $\mu^{+}$spin-lattice relaxation rate $\lambda$ should be proportional to $x^{2}$ at high temperatures, whereas $\lambda$ a $x$ for the RKKY process. 15 At $100 \mathrm{~K}$ our reasured values of $\lambda_{1}$ for $x>0.35$ are proportional to $x^{2}$, which indicates that the Korringa mechanism is dominant in this temperature regime. The Korringa mechan1sm has also been observed by NMR in $\operatorname{SmRh}_{4} B_{4}$ at high temperatures. 16 For $x>0.35$ and $11 \mathrm{~K}<\mathrm{T}<50 \mathrm{~K}$ the $\mu^{+}$ 
relaxution rate is too large to be observed, 5 so that the cencentration dependence of $\lambda_{1}$ cannot be determined in this tempergture range.

Quasistatic motion of $h_{L}(t)$ sets in for temperatures below $\sim 11 \mathrm{~K}$ for $x=0.7$, as previously reported, ${ }^{5}$ as well as in the present case $x=0.02$. This concentration dependence is additional confirmation that the dramatic reduction in $\mathrm{Ho}^{3+}$ fluctuation rate is due to the CEF ground-state 1solation, which is a single-ton effect and should not depend greatly on concentration. We have calculated CEF energies and wave functions for $\mathrm{RE}^{3+}$ lons in the $\operatorname{RERh}_{4} \mathrm{~B}_{4}$ structure by diagonalizing the hamiltonian for $42 \mathrm{~m}$ point symmetry, using the crystal-fleld parameters of Dunlap and Niarchos. 17 For $\mathrm{Ho}^{3+}$ the ground and first exclted states are each doubly degenerate, with nearly pure $\left|J_{z}= \pm\right\rangle$ and $| \pm 7\rangle$ wave functions respectively. The first exc1ted states occur at $\delta \equiv 55 \mathrm{~K}$. Allowed transitlons from $| \pm 8\rangle$ to $|\mp 8\rangle$ can therefore ocsur only through small admixtures of different $J_{z}$ elgenfunctions in the ground and excited states, which are separated by at least 55 K. The slow $\mathrm{Ho}^{3+}$-moment fluctuations at low temperatures can then be accounted for by a combinazion of the large ground-state isolation and the weakness of $\Delta \mathrm{J}_{2}= \pm 1$ transitions within the ground-state doublet.

Below $\sim 9 k$ the values of $\lambda_{2}$ for $x=0.02$ and $x=0.7$, shown in Fig. 2, are remarkably similar considering the large difference in Ho concentratione. This observation can be understood if the dominant $\mathrm{Ho}^{3+}$-moment relaxation mechanism is again Korringa scattering, as at $100 \mathrm{~K}$, because this mechanism would result in a concentration-independent correlation time $\tau_{m}$ for the $\mu^{+}$local flelds. Since $\lambda_{2} \sim 1 / \tau_{m}$ for quasistatic fluctuations (Sect. II.C), $\lambda_{2}$ would also be independent of $\underline{x}$, as the data of Fig. 2 indicate. At least one source of difference in the temperature dependence of $\lambda_{2}$ between $x=0.02$ and $x=0.7$ is the onset of ferromagnet 1sm below the 
Curle temperature $T_{c}=4.1 \mathrm{~K}$ at the latter concentration. This is presumably respons1ble for the "knee" in $\lambda_{2}(T, x=0.7)$ near $T_{c}$.

It should be noted that most of these data are for temperatures below the superconducting transition temperature $T_{6}$. The anomalous bahavior of $\lambda_{2}$ in the superconducting state, 5 although not we11. understood, can therefore be attributed to $\mathrm{Ho}^{3+}-\mathrm{sp} 1 \mathrm{n}$ relaxation by scattering of excitations $1 \mathrm{n}$ the superconducting conduction-electron system.

Kumagal and Fradin 18 have reported MMR studies of very dilute alloys of Gd and EI in $\mathrm{YRh}_{4} \mathrm{~B}_{4}$, whlch indicate that the RKKY process dominates RE fluctuations in these alloys for $T=T_{s}$. The low-lying CEF states of these

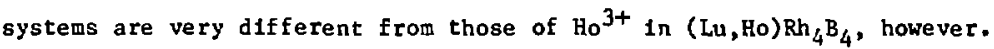

Below $T_{s}$ the temperature dependence of $\lambda_{2}$ for $x=0.02$ indicates an activation energy $\Delta=10 \mathrm{~K}$, which is much smaller than the CEF ground-stace Isolation $\delta$. Since $\Delta \approx 1.8 \mathrm{~T}$, Interpietation of $\Delta$ as a superconducting gap parameter yields a value consideraby smaller than the BCS gap parameter $\Delta_{\mathrm{BCS}}=3.5 \mathrm{~T}_{\mathrm{s}}$. Such an interpretation 1s, however, speculative at this time, because it is not clear how the superconducting state could reduce the activation energy for $\mathrm{Ho}^{3+}$ fluctuations to a value much less than $\delta$. Further ${ }_{\mathrm{S}} \mathrm{SR}$ experiments in dilute (RE) $\mathrm{Rh}_{4} \mathrm{~B}_{4}$ alloys are under way to Investigate this phenomenon.

*Work supported by the U.S. Department of Energy, U.S. National Sclence Foundation Grants DMR-7909223 and DMR-8115543, and the U.C. RIvers1de Committee on Research.

tPermanent address: Laboratolre d'Interactions Hyperfines, C.E.N.G., B.P. $85 x, 38041$ Grenoble, France.

Fermanent address: Tata Institute of Fundamental Research, Bombay, India. 


\section{REFERENCES}

1. P. W. Anderson and H. Suhl, Phys. ReV. 116, 898 (1959); L. P. Gorkov and A. I. Rusinov, Sov. Phys.JETP 19, 922 (1964).

2. B. T. Matthlas, E. Corenzw1t, J. M. Vandenberg, and E. Barz, Proc. Nat1. Acad. Sc1. USA 74, 1334 (1977).

3. See e.g. Proc. Int. Conf. on Ternary Superconductors, tdited by G. K. Shency; B. D. Dunlap, and F. Y. Fradln (North Holland, New York, 1981).

4. M. B. Maple, H. C. Hamake r, D. C. Johnson, H. B. McKay, and L. D. Woolf, J. Less-Comm. Met. 62, 251 (1978).

5. C. Boekema, R. H. Heffner, R. L. Hutson, M. Leon, M. E. Schillaci, J. L. Smlth, S. A. Dodds, D. E. MacLaugh11n, J. Appl. Phys. 53, $2625(1982)$

6. D. E. MacLaughlin, S. A. Dodds, C. Boekema, R. H. Heffner, R. L. Hutson, M. Leon, M. E. Schillac1, and J. L. Smith, J. Magn. Magn. vat. 31-34, 497.

7. R. S. Hayano, Y. J. Uemura, J. Imazato, N. Nishida, T. Yamazaki, and R. Kubo, Phys. Rev. B 20, 850 (1979).

8. R. Kubo and T. Toyabe, In Magnet1c Resonance and Relaxation, ed. by R. Blinc (North Hollanc, Amsterdam, 1967) p. 810 .

9. Y. J. Uemura, Hyperf. Int. $\underline{\text {, }} 739$ (1980).

10. R. H. Heffner, M. Leon, M. E. Schlllac1, D. E. MacLaughlin, and S. A. Dodd6, J. Appl. Phys. 53, 2174 (1982).

11. R. E. Walstedt and L. R. Walker, Phys, Rev. B 9, 4857 (1974). 
12. A. Schenck, Helv. Phys. Acta 54, 471 (1981).

13. H. R. ott, L. D. Woolf, M. B. Maple, and D. C. Johnson, J. Low Temp. Phys. 39, 383 (1980).

14. H. Hasegawa, Prog. Theor. Phys, 21, 483 (1959).

15. M. R. Metenry, B. G. Silbernagel, and J. H. Wernick, Phys. Rev. B 5. $2958(1972):$

16. K. Kumaga1, Y. Inoue, K. Kohori, and K. Asayama, in Ref. 3, p. 185.

17. B. D. Dunlap and D. NLarchos, Sol. St. Come 44, 1577 (1982). The actual parameters used vary somewhat from this reference, and were abtained from B. D. Dunlap, private communication.

18. K. Kumaga1 and F. Y. Fradin, Phys. Rev. B 27, 2770 (1982). 


\section{FIGURE CAPTIONS}

FIG. 1. Zeroffeld aron $\operatorname{spln}$ relaxation function $G_{z}(t)$ at representative temperaturee in $\mathrm{Ho}_{0.02^{\mathrm{Lu}}} 0.98^{\mathrm{Rh}} 4^{\mathrm{B}} 4^{\circ}$. The curves are fits to the appropriate functions as discussed in the text.

FIG. 2. Temperature dependence of the zero-field $\mu^{+-}$spin-1attice relaxation rate 1n Ho $\mathrm{Hu}_{1-\mathrm{x}} \mathrm{Rh}_{4} \mathrm{~B}_{4}, \mathrm{x}=0.02$ (c1rcles and solld curves) and 0.7 (trlangles and dashed curves). Open symbols: relaxation rate of the full $\mu^{+}$asymetry (relaxation rate $\lambda_{1}$ ). Filled symbols: relaxation rate of the reduced-asymetry component of the $\mu^{+}$polarization below $\sim 11 \mathrm{~K}$ (relaxecion rate $\lambda_{2}$ ). Superconducting $\left(T_{s}\right)$ and ferromagnetic $\left(T_{c}\right)$ trangition temperatures are indicated. The curves are guldes to the eye. 


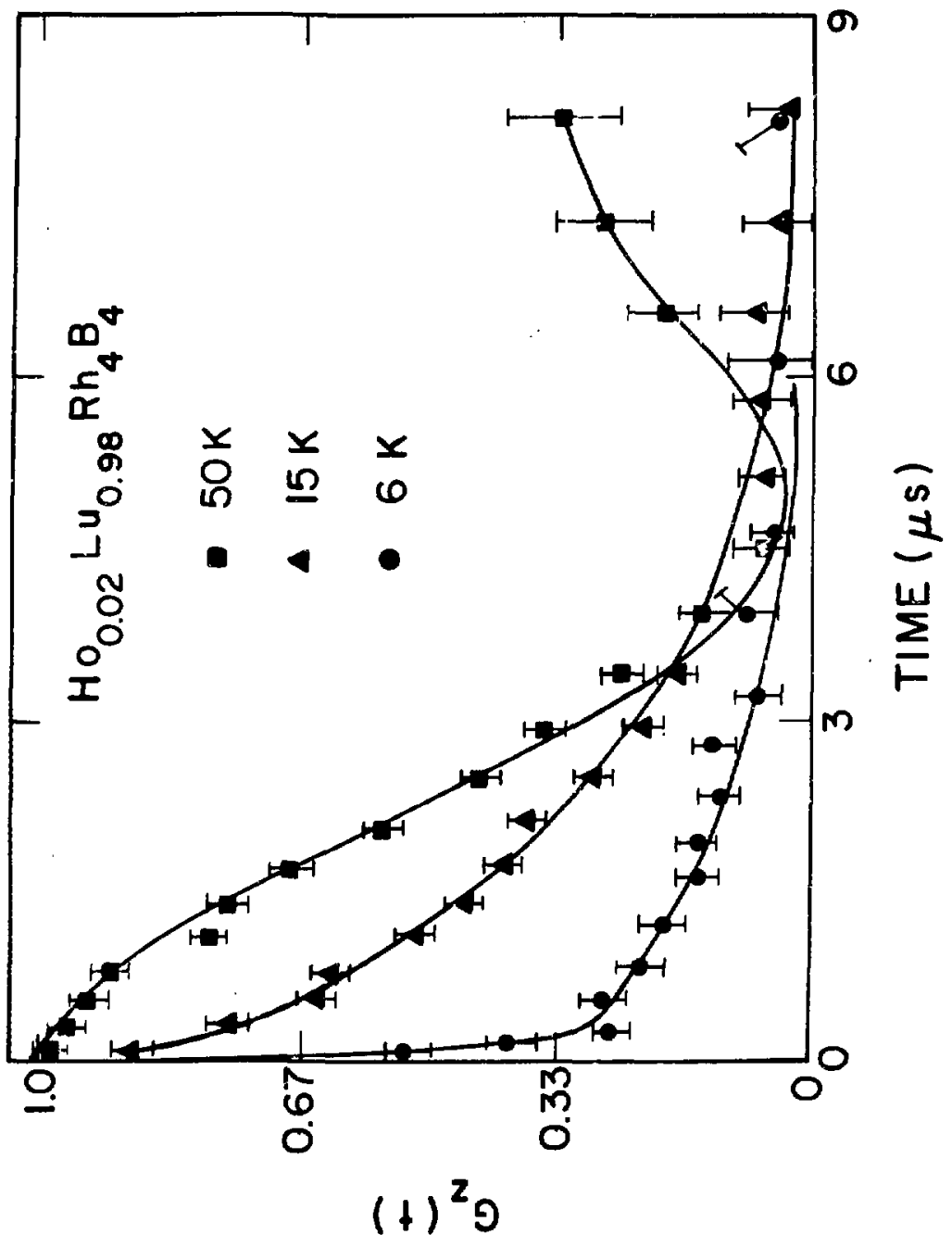


$T(K)$

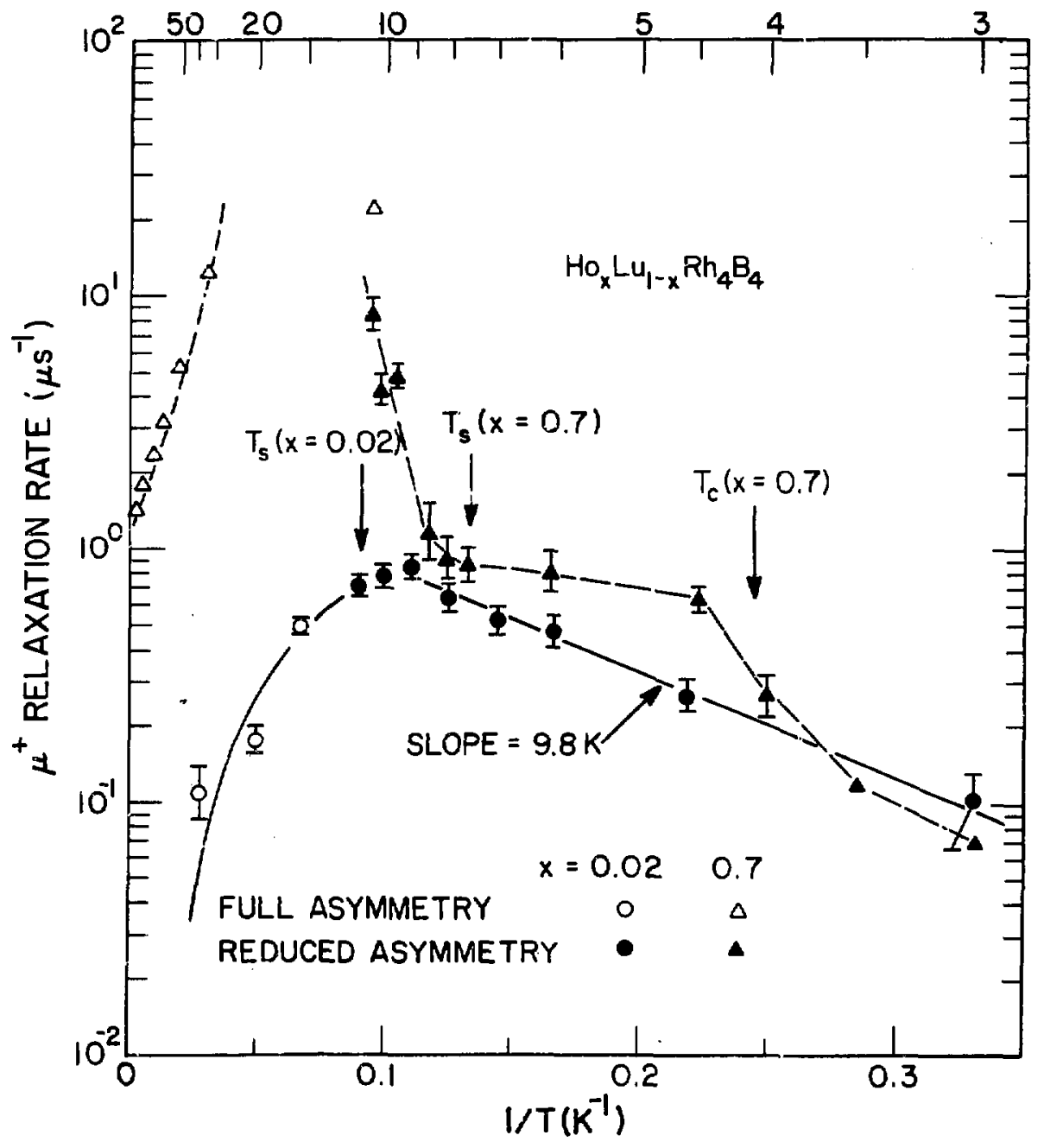


MUON SPIN RELAXATION IN SPIN GLASS PdMn

R. H. Heffner, M. Leon, M. E. Schillaci

Los Alamos National Laboratory

Los Alamos, NM 87545

S. A. Dodds, G. A. Gist

Department of Physics, Rice University

Houston, TX 77251

D. E. Mactaughlin

Department of Physics, University of California

Riverside, CA 92521

J. A. Mydosh, G. J. Nieuwenhuys

Kamerlingh Onnes Laboratory, University of Leiden

The Netherlands

ABSTPACT

Nuon spin relaxation ( $\mu S R$ ) rates have been measured in transverse, longitudinal, and zero applied field for the spin glass Pdis ( 7 at. $\left.\frac{9}{5}\right)$, and are compared with a previous study of the disordered ferromagnet PdMn (2 at. 8 ). The calculated paramagnetic state transverse field relaxation rate for non-interacting spins is much larger than the observed rate in spin glass Fam, but is in good agreement with ferromagnetic PdM. The zero field relaxation rate shows a sharp cusp at $T_{g}=5 \mathrm{~K}$. An applied longitudinal field of $5 \mathrm{kG}$ is insufficient to suppress this cusp in spin glass PdMn, but will suppress a similar cusp in ferromagnetic PdMn. Below $\mathrm{T}_{\mathrm{g}}$, a distribution of quasistatic local fields is observed in zero field, which has the same temperature dependence for both samples. Comparisons with model calculations are discussed. 
I. Introduction

Spin glass PdMn has been widely studied as an example of a non-RKKY spin glass. 1,2 A competition between direct anti-ferromagretic couplings and long range ferromagnetic couplings leads to a complicated phase diagram with a ferromagnetic phase for $c\{3$ at. $z$, and a spin $\dot{g}$ lass phase for $c \grave{\imath} 5$ at. $z$ If this work, muon spin relaxation ( HSR) is used as a microscopic probe of the ordering in spin slass PdMn (7 at. 8 ). The main goal of this work is to measure the temperature and field cependence of both the quasistatic local field distribution at the muon site and the muon's dynamic spin lattice relaxation. Theories of linewidths and relaxation mechanisms in dilute alloys are used to infer features of the impurity spin configuration. Comparisons with a previous study of ferromagnetic PdMn (2 at. 8$)^{4}$ are used to show how the difference in magnetic ordering brought about by a charge in concentration influences microscopic properties.

The experiments were carried out at the stopped Muon Channel of the Clinton P. Anderson Meson Physics Facility (LAMPF) at the LOS Alamos National Iaboratory, using a standard $4 \Omega R$ spectrometer. 5 The saiple was in the form of a polycrystalline disk $3 \mathrm{~mm}$ thick and $30 \mathrm{~mm}$ in diameter; preparation techniques have been described in an earlier paper. 4 Ac susceptibility measurements showed a cusp at $T_{g}=5 K$, consistent with the system phase diagram. ${ }^{6}$

II. Quasistatic Field Distributions

Figure 1 shows measured transverse field exponential relaxation rates for applied fields of $200 \mathrm{G}, 1 \mathrm{kG}$, and $5 \mathrm{kG}$. Measured spin lattice relaxation rates (discussed below) indicate that 
the non-secular contribution to $\lambda_{1}$ is small. In addition, measurements of muon diffusion in. Pd with Gd impurities indicate a negligible diffusion rate below about $100 \mathrm{~K} .{ }^{7}$ Thus the rates shown in figure 1 represent only the quasistatic field distribution in PdM .

Walstedt and Walker ${ }^{8}$ (WW) have shown that if the local environment around the muon consists of non-interacting and equivalent, rapidly fluctuating spins distributed at random at concentration $c$, then in the dilute limit the relaxation is exponential with rate

$$
\lambda_{1}=\lambda_{10}<S_{2}>
$$

where $\left\langle S_{z}\right\rangle$ is the thezinal average of the $z$ component of the impurity spin. If the impurity spins are quasistatic (slow fluctuations) then this expression becomes

$$
\lambda_{2}=\lambda_{10}\left\langle\left|s_{2}\right|\right\rangle \text {. }
$$

For pure dipolar coupling,

$$
\lambda_{\perp O}=\frac{\delta \pi^{2}}{9 \sqrt{3}} N_{O} C \gamma_{\mu} g \mu_{B}
$$

where $N_{O}$ is the density of impurity sites, $v_{\mu}$ is the muon gyromagnetic ratio, and $\mathrm{g}$ is the impurity spin $\mathrm{g}$ factor. These expressions are for the case of a large applied field, so that only components of impurity spins parallel to the applied field contribute to the 2 component of the local field distribution at the muon.

The observed paramagnetic susceptibility in a Pdkn (5 at. \&) sample is well described by a $g=2, s=5 / 2$ curie law for $T 23 \mathrm{~T} g$ 
and low field, ${ }^{1}$ so that using this Curie law for $\left\langle s_{z}\right\rangle$ in equation (1) gives the lines shown in Figure l. At low temperatures, we simulate the spin glass state by assuming spins to be quasistatic and equally distributed among the $2 S+1 \mathrm{M}_{S}$ states in calculating the orcer parameter $\left\langle\left|s_{z}\right|>\right.$. Equation (2) then gives the high field frozen spin linewidth

$$
\left(\lambda_{\perp}\right)_{\text {frozen }}=57.1 \mathrm{\mu s}^{-1} \text { (high field). }
$$

A concentration of 7 at. 8 is not ordinarily considered to be in the dilute limit, so we have calculated a polycrystalline averaged lineshape numerically; the result differs in width from the WW result by no more than 78 .

The discrepancy in figure 1 between the ', data and the Ww prediction using a Curie law well above $T_{g}$ is not understcod. Similar data on the disordered ferromagnet pdM (2 at .8) 4 show very good agreement with the wh result. We conclude that the observed discrepancy is due to the spin glass ordering, and is present for $T$ well above $\mathrm{T}_{\mathrm{g}}$. Transverse field $\mu S R$ results in spin glass AgM show an increase above the WW result to at least $10 \mathrm{~T}_{\mathrm{g}}{ }^{9}$

In zero applied field, the wW result is no longer vilid. since all three components of the local field distribution contribute to the muon relaxation. Mikaelyan and Smilsalo have generalized the WW expression to the case of zero field relaxation in a spin glass. The low temperature spin glass state is simulated by assuming quasistatic classical impurity spins, each of length $s$ 
and oriented along a randomly chosen local field direction. The resulting muon local field distribution is Lorentzian, with width

$$
a=0.827 \lambda_{\perp_{0}} S
$$

which is in good agreement with the Monte carlo simulation of Fiory. 11 For PaMn (7 at. \%), equation (5) gives a zero field frozen spin linewidth

$$
a_{0}=78.8 \mathrm{Hs}^{-1} \text { (zero field). }
$$

The zerofield muon relaxation furction allows a separation between a quasistatic field distribution of width a, and relaxation due to dynamic spin lattice relaxation processes. If we assume the fluctuation rate to be fast compared with a, then the long time decay will be a 'root exponential. 12 Thus for fast dynamic fluctuations, the zero field relaxation function is

$$
G_{z}(t)=\frac{1}{3} \exp \left(-\left(\lambda_{\|} t\right)^{1 / 2}\right)+\frac{2}{3}(1-a t) \exp (-a t)
$$

The measured zero field relaxation data were fit to equation (7) for the width of the guasistatic local field distribution (a) below $\mathrm{T}_{\mathrm{g}}$. The temperature dependence of a is shown in figure 2 , normalized to $a_{0}$. The scaled quasistatic width in ferromagnetic PoMn $(2 \text { at. \% })^{4}$ is also shown in figure 2 . The similar temperature dependence in the two cases indicates that the development of a static field distribution is independent of the character of the magnetic ordering. 
III. Spin Lattice Relaxation

Figure 3 presents measured muon spin lattice relaxation rates in zero and applied longitudinal field above and below $\mathrm{T}_{g}$. Above $T_{g}$ ' the relaxation was fit to the 'root exponential' form appropriate for rapidly fluctuating (paramacnetic) spins in a dilute alloy, 12

$$
G_{z}(t)=\exp \left(-\left(\lambda_{\mid i} t\right)^{1 / 2}\right)
$$

with rates plotted in figure 3. Fits of the data to a simple exponential form for $G_{z}(t)$ yield a similar chi-squared value, so that we have not explicity demonstrated the root exponential form. Below $\mathrm{T}_{\mathrm{g}}$, we expect that fluctuations will remain rapid, since this has been observed by USR in spin glass AgMn. 13 Thus a root exponential decay of the $1 / 3$ component (equation (7) was used to fit the zero field data for $\lambda_{1,1}$.

The peak in the zero field spin lattice relaxation rate: at $T_{g}$ is interpreted as being due to a change in the power spectruof Mn spin fluctuations near the glass transition. Other uSR measurements in.spin glass system (모, Cunin) 13,14 show a sinilar dramatic change in the Mn spin fluctuations around $\mathrm{T}_{\mathrm{g}}$. Note that a relaxation mechanism is still effective near $T_{g}$ in an applied field of $5 \mathrm{kG}$, whereas in ferromagnetic pdMn (2 at. 8) an applied field of $5 \mathrm{kG}$ completely suppresses the fluctuation induced relaxation. 4

At temperatures well below $\mathrm{T}_{g^{\prime}}$ spin lattice relaxation is presumably due to low energy excitations of the ground state. 15 
16

A calculation of the spectrum of excitation frequencies for spin glass pdMn(10 at. \%) has shown a complicated three peak structure, with the mean spectral weight at a frequency $\sim 5 \times 10^{12} \mathrm{~s}^{-1}$. Since these fluctuations are much too fast to cause depolarization via the dipolar interaction in the lifetime of the muon, the observed relaxation must be due to some other, lower frequency component of the excitation spectrum. Recent analytic theories of spin wave excitations in spin glasses predict that small k magnons can propagate through the system with a dispersion relation linear in $k .17$ These excitations are at Erequencies lower than the localized modes, and may be responsible for the relaxation observed at low temperatures. Future zero field iSR studies in spin glasses at lower temperatures may provide useful data on relaxation for comparison with these theories.

IV. Conclusions

The transverse field relaxation rate $\lambda_{\perp}$ is not proportional to the ubserved Curie law magnetization in PdM ( 7 at. 8 ) for temperatures as high as $100 \%\left(20 \mathrm{~T}_{g}\right)$, where the onset of muon diffusion makes interpretation of $\lambda$, difficult. This result is especially surprising since: $\lambda_{1}$ in ferromagnetic PdMn ( 2 at. 8 ) is proportional to a Curie-Weiss magnetization over a wide temperature range, indicating that the observed reduction in $\lambda_{\perp}$ below 
the Curie law prediction may be a high temperature precursor of the spin glass phase. We know of no satisfactory explanation for this effect nor for the increase in $\lambda_{\perp}$ above the measured magnetization in spin glass AgMn (1.6 at. 8).

In zero field, we observe the onset of a quasistatic field distribution below $\mathbf{T}_{\mathrm{g}}$ with a similar temperature dependence to that observed in PdMn (2 at. 8). Spin lattice relaxation near $\mathrm{T}_{\mathrm{g}}$ shows a strong cusp, indicating a change in the spectrum of Min spin fluctuations. These fluctuations continue to induce relaxation in a large applied field, in contrast to PdMn (2 at. 8 ).

Acknriwledgements

We are grateful for helpful discussions with R. L. Hutson. This work was supported by the Department of Energy, the National Science Foundation (Grant Nos. DMR79-09223 and DMR81-15543) and the Netherlands "stichtung voor Fundamenteel onderzoek der Materie" (FOM). 
FIGURE CAPTIONS

Figure 1. Temperature anc field dependence of the transverse muon relaxation rațe $\lambda_{1}$ in pdMn (7 at. 8$)$. Circles: 200 G. Triangles: 1 kG. Squares: 5 kG. The solid lines are from equation (1) using a curie law for $\left\langle s_{z}\right\rangle \cdot\left(\lambda_{\perp}\right)_{f}$ is the frozen spin rate (equation (4)).

Figure 2. Reduced temperature dependence of the zero field quasistatic field distribution width a(T), normalized to the theoretical zero field frozen rate $a_{0}$. Open circles: PdMn $(2$ at. 8$), a_{0}=28 \cdot \mu \mathrm{s}^{-1}, \mathrm{~T}_{\mathrm{C}}=5.8 \mathrm{~K}$. Filled circles: $\underline{\text { pdM }}(7 \mathrm{at} .8), \mathrm{a}_{0}=78.8 \mathrm{\mu s}^{-1}$, $T_{g}=5 . K$.

Figure 3. Temperature and field dependence of the muon spin lattice relaxation rate. Circles: zero field. Triangles: $200 \mathrm{G}$. Squares: $5 \mathrm{~kg}$. Fitting functions used are described in the text. 


\section{REFERENCES}

1. B. R. Coles, H. Jamieson, R. H. Taylor, A. Tari, J. Phys, F 5. $565(1975)$.

2. 5. C. Ho, I. Maartense, G. Williams, J. Phys. F 11, 1107 (1981).

3. H. A. Zweers, G. J. van den Berg, J. Phys, F 5,555 (1975).

4. S. A. Dodds, G. A. Gist, D. E. Mactaughlin, R. H. Heffner, M. Leon, M. E. Schillaci, G. J. Nieuwenhuys, J. A. Mydosh, Phys. Rev. B, in press.

5. E. Karlsson, Phys. Reports 82, 271 (1982).

Б. J. A. Mydosh, G. I. Nieuwenhuys, B. H. Verbeek, Phys. Rev. B 20, 1280 (1979).

7. S. A. Dodds, R. T. Stein, R. H. Heffner, R. L. Hutson, M. Leon, M. E. Schillaci, D. E. Nactaughlin, J. A. Mydosh, G. J. Nieuwenhuys, P. M. Richards, Bull. Am. Phys. Soc. 27, 162 (1982).

8. R. E. Walstedt, L. R. Walker, Phys. Rev. B 9, 4857 (1974).

9. J. A. Brown, R. H. Heffner, T. A. Kitchens, M. Leon, C. E. Olsen, M. E. Scirillaci, S. A. Dodds, D. E. MacLaughlin, J. Appl. Phys. 52, 1766 (1981); R. H. Heffrer, M. Leon, M. E. Schillaci, D. E. Maclaughlin, S. A. Dodds, J. Appl. Phys. 53, 2174 (1982). 
10. A. L. Mikaelyan, V. P. Smilga, Sov. Phys. JETP 53, 1216 (1981).

11. A. T. Fiory, Hyp. Int. 8,777 (1981).

12. M. R. McHenry, B. G. Silbernagel, J. H. Wernick, Phys. Rev. B ‥ $2958(1972)$.

13. R. H. Heffner, M. Leon, M. E. Schillaci, D. E. MacLaughlin,

S. A. Dodds, J. Appl. Phys. $\underline{53}, 2174$ (1982).

14. X. J. Uemura, T. Yamazaki, R. S. Hayano, R. Nakai, C. Y. Huang, Phys. Rev. Lett. 45,583 (1980).

15. D. Beeman, P. Pincus, Phys. Rev. $\underline{166}, 359$ (1968); A. H. Mitchell, J. Chem. Phys. 27, 17 (1957).

16. W. Y. Ching, D. L. Huber, B. H. Verbeek, J. Appl. Phys. 50, $1715(1979)$.

17. B. I. Halperin, W. M. Saslow, Phys. Rev. B 16, 2154 (1977); w. M. Saslow, Phys. Rev. B 22, 1174 (1980); K. H. Fischer, 2. Ehys. B 39,37 (1980); S. E. Barnes, J. Phys. F 11, I249 (1981). 


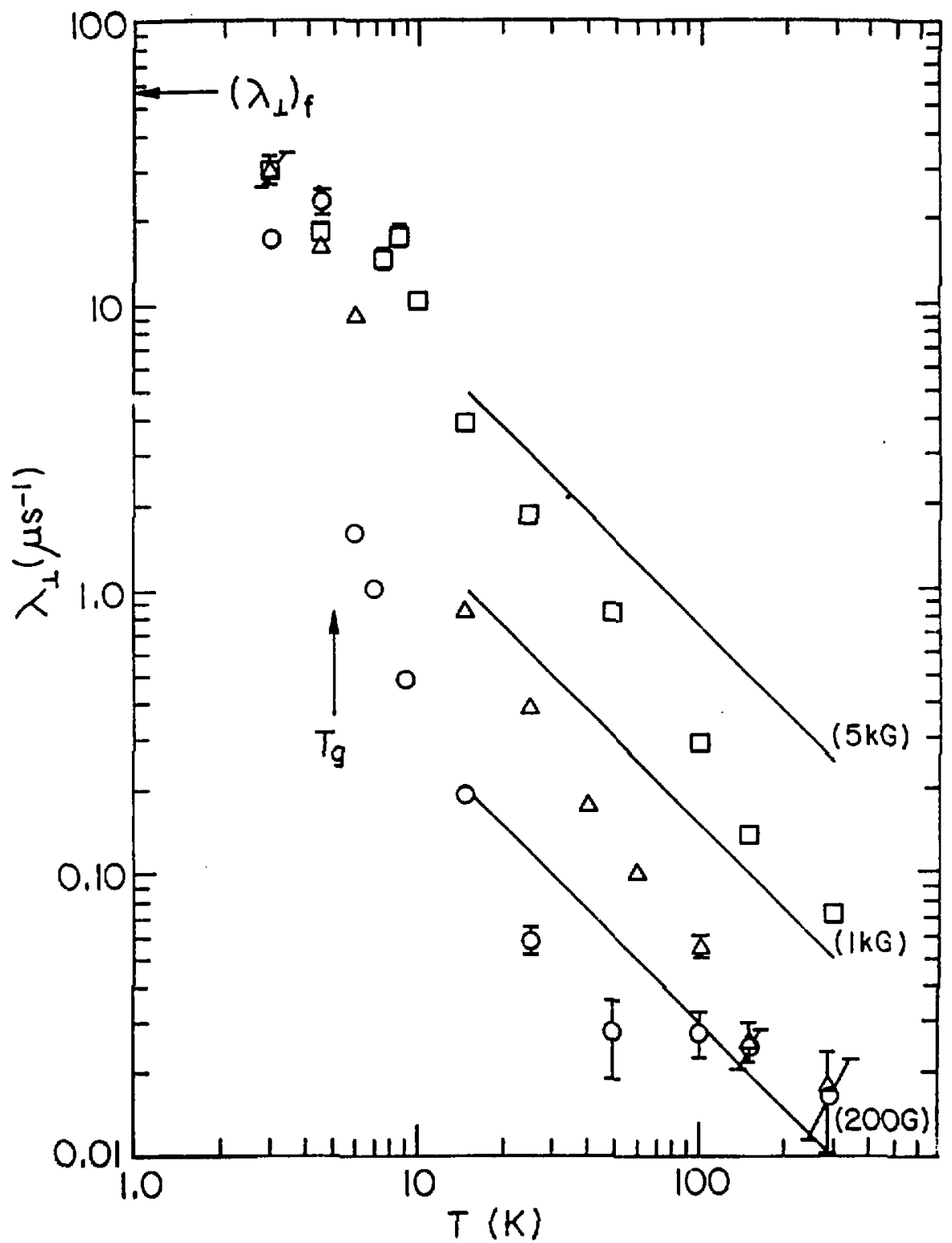

Figure 1 


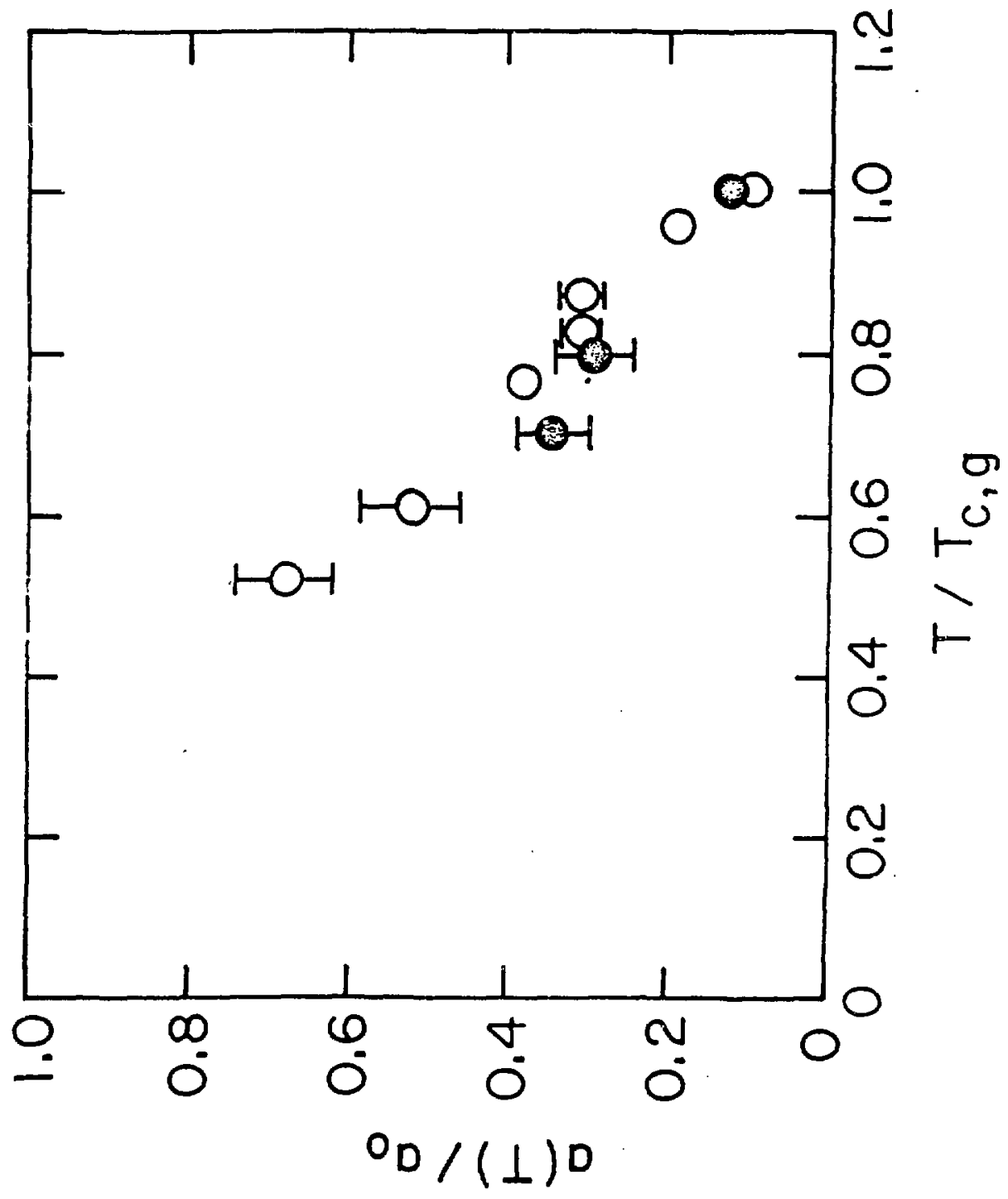

Figure 2 


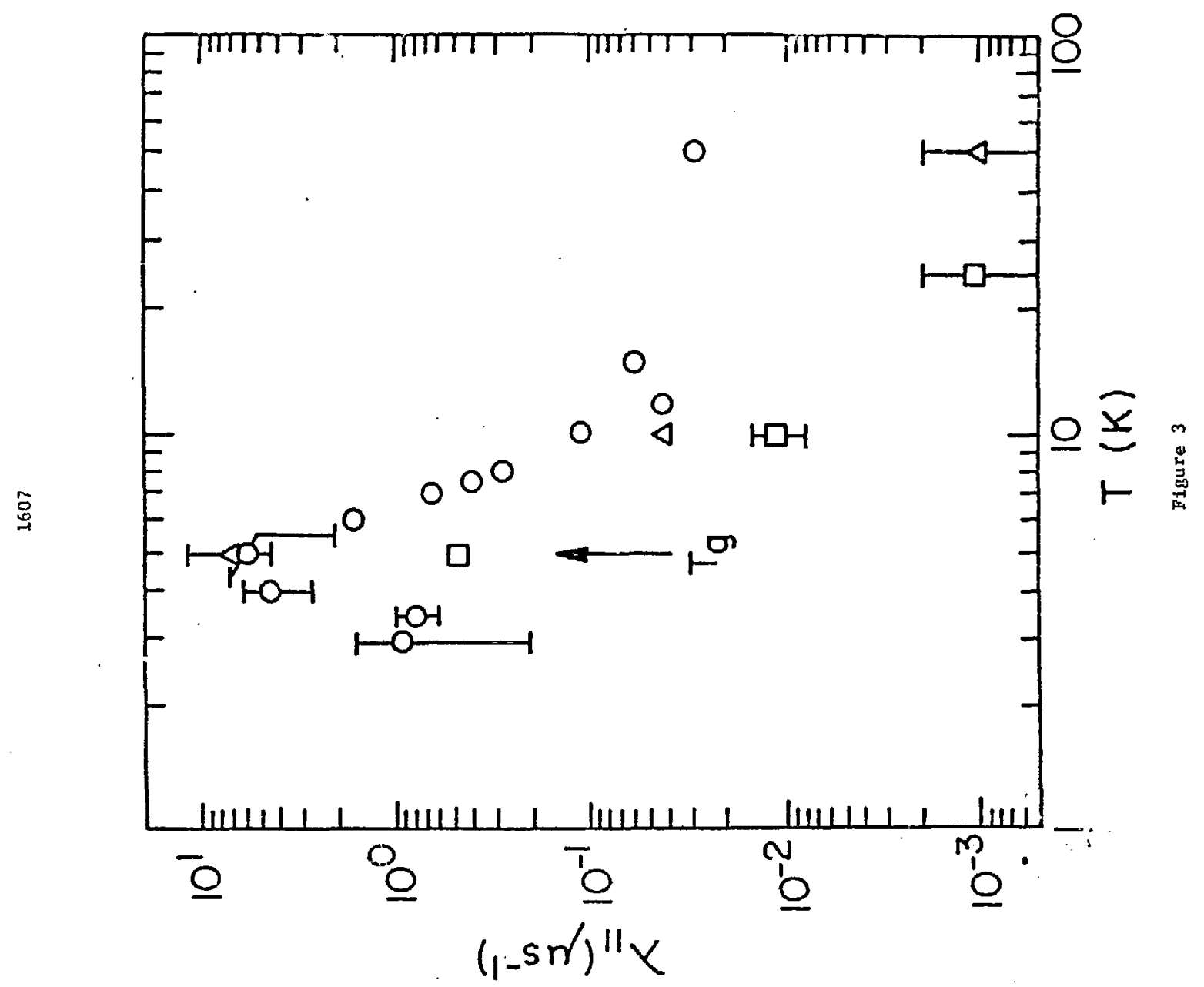

